Supporting Information for

\title{
Preparation of highly substituted 6-arylpurine ribonucleosides by Ni-catalyzed cyclotrimerization. The scope of the reaction.
}

Pavel Turek, Petr Novák, Radek Pohl, Michal Hocek, and Martin Kotora

\section{Contents}

I. General Methods. $\quad$ S - 1

II. Synthesis of Protected 9-( $\beta$-D-Ribofuranosyl)-6-alkynylpurines 1. S - 2

III. Synthesis of Protected 9-( $\beta$-D-Ribofuranosyl)-6-arylpurines 3. S - 4

IV. Deprotection of the Acetylated 9-( $\beta$-D-Ribofuranosyl)-6-arylpurines. $\quad$ S - 15

V. Cyclotrimerization of Unprotected 9-( $\beta$-D-Ribofuranosyl)-6-alkynylpurines. S - 20

$\begin{array}{ll}\text { VI. References. } & \text { S - } 21\end{array}$

VII. Spectra of the Prepared Compounds. $\quad$ S - 22

\section{General Methods.}

All solvents were used as obtained unless otherwise noted. THF and toluene were distilled from sodium and benzophenone, and acetonitrile from $\mathrm{CaH}_{2}$ under Ar. Starting diynes 2 that were not commercially available were prepared by standard procedures by the reaction of propargyl bromide with the corresponding $C$-acids under basic conditions. ${ }^{1}$ $\mathrm{CoBr}\left(\mathrm{PPh}_{3}\right)_{3}{ }^{2}$ and $\mathrm{NiBr}_{2}\left(\mathrm{PPh}_{3}\right)_{2}{ }^{3}$ were prepared according to the previously reported procedures. All other reagents were obtained from commercial sources. ${ }^{1} \mathrm{H}$ (400 or 500 $\mathrm{MHz}$ ) and ${ }^{13} \mathrm{C}$ NMR (100.6 or $125.8 \mathrm{MHz}$ ) spectra were recorded as solutions in $\mathrm{CDCl}_{3}$ with $\mathrm{Me}_{4} \mathrm{Si}$ as an internal standard or as solutions in DMSO- $d_{6}$ referenced to the residual solvent signal. Chemical shifts are given in $\delta$-scale, coupling constants $J$ are given in Hz. Melting points (uncorrected) were determined using a Kofler apparatus. Infrared spectra were recorded as $\mathrm{CHCl}_{3}$ solutions or as $\mathrm{KBr}$ tablets and are reported in wave numbers $\left(\mathrm{cm}^{-1}\right)$. Fluka 60 silica gel was used for flash chromatography. TLC was performed on silica gel 60 $\mathrm{F}_{254}$-coated aluminum sheets. All cyclotrimerization reactions were carried out under an argon atmosphere using Schlenk-tube technique. 


\section{Synthesis of Protected 9-( $\beta$-D-Ribofuranosyl)-6-alkynylpurines 1.}

\section{General procedure for cross-coupling reactions of 6-chloropurine with acetylenes.}

Triethylamine $(4 \mathrm{ml})$ and DMF $(20 \mathrm{ml})$ were added to an argon purged mixture of 6-chloro-9(2,3,5-tri-O-acetyl- $\beta$-D-ribofuranosyl)purine (3.7 g, $9 \mathrm{mmol})$, CuI (104 mg, $0.54 \mathrm{mmol}$ ), $\left[\mathrm{PdCl}_{2}\left(\mathrm{PPh}_{2}\right)_{2}\right](128 \mathrm{mg}, 0.18 \mathrm{mmol})$, acetylene $(10.8 \mathrm{mmol})$ and the mixture was stirred at 60 ${ }^{\circ} \mathrm{C}$ for $6 \mathrm{~h}$. Solvents were evaporated under reduced pressure and the residue was chromatographed on silica gel column.

\section{6-(Phenylethynyl)-9-(2,3,5-tri-O-acetyl- $\beta$-D-ribofuranosyl)-9H-purine (1a).}

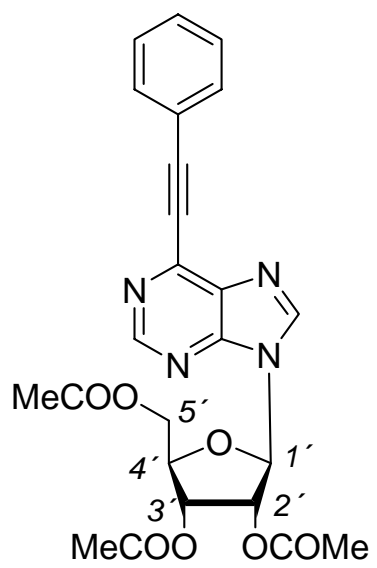

Column chromatography on silica gel (hexane/EtOAc 1/2) afforded $3.5 \mathrm{~g}(80 \%)$ of a yellowish oil, which after co-evaporation with diethyl ether formed a white foam. Experimental data were in accordance with the published data. ${ }^{4}$

6-(Hex-1-yn-1-yl)-9-(2,3,5-tri-O-acetyl-ß-D-ribofuranosyl)-9H-purine (1b).

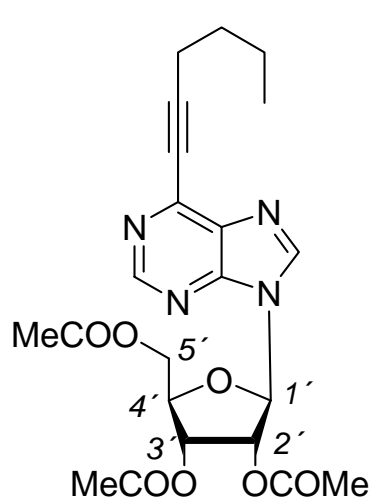

Column chromatography on silica gel (hexane/EtOAc 2/3) afforded $3.3 \mathrm{~g}(80 \%)$ of a yellowish oil: $[\alpha]_{\mathrm{D}}-19.4\left(\right.$ c $\left.0.24, \mathrm{CHCl}_{3}\right),{ }^{1} \mathrm{H} \mathrm{NMR}$ $\left(400 \mathrm{MHz}, \mathrm{CDCl}_{3}\right) \delta 0.96\left(\mathrm{t}, 3 \mathrm{H}, J_{\text {vic }}=7.3, \mathrm{CH}_{3} \mathrm{CH}_{2} \mathrm{CH}_{2} \mathrm{CH}_{2^{-}}\right), 1.53$ and $1.71\left(2 \times \mathrm{m}, 2 \times 2 \mathrm{H}, \mathrm{CH}_{3} \mathrm{CH}_{2} \mathrm{CH}_{2} \mathrm{CH}_{2}-\right), 2.08,2.12$ and $2.16(3 \times \mathrm{s}$, $\left.3 \times 3 \mathrm{H}, \mathrm{CH}_{3} \mathrm{CO}\right), 2.61\left(\mathrm{t}, 2 \mathrm{H}, J_{\text {vic }}=7.2, \mathrm{CH}_{3} \mathrm{CH}_{2} \mathrm{CH}_{2} \mathrm{CH}_{2^{-}}\right), 4.35-4.50$ (m, 3H, H-4' and H-5'), $5.67\left(\mathrm{dd}, 1 \mathrm{H}, J_{3^{\prime}, 2^{\prime}}=5.5, J_{3^{\prime}, 4^{\prime}}=4.4, \mathrm{H}-3^{\prime}\right)$, $5.96\left(\mathrm{t}, 1 \mathrm{H}, J_{2^{\prime}, 3^{\prime}}=5.5, J_{2^{\prime}, 1^{\prime}}=5.3, \mathrm{H}-2^{\prime}\right), 6.24\left(\mathrm{~d}, 1 \mathrm{H}, J_{1^{\prime}, 2^{\prime}}=5.3, \mathrm{H}-\right.$ $\left.1^{\prime}\right), 8.25$ (s, 1H, H-8), 8.91 (s, 1H, H-2); ${ }^{13} \mathrm{C}$ NMR (100.6 MHz, $\left.\mathrm{CDCl}_{3}\right) \delta 13.56\left(\mathrm{CH}_{3} \mathrm{CH}_{2} \mathrm{CH}_{2} \mathrm{CH}_{2}-\right), 19.63\left(\mathrm{CH}_{3} \mathrm{CH}_{2} \mathrm{CH}_{2} \mathrm{CH}_{2}-\right), 20.34,20.50$ and 20.72 $\left(\mathrm{CH}_{3} \mathrm{CO}\right), 22.10$ and $30.09\left(\mathrm{CH}_{3} \mathrm{CH}_{2} \mathrm{CH}_{2} \mathrm{CH}_{2}-\right), 62.95\left(\mathrm{CH}_{2}-^{\prime}\right), 70.55\left(\mathrm{CH}-3^{\prime}\right), 73.04$ (CH-2'), 75.89 (Pur-C $\equiv \mathrm{C}-\mathrm{Bu}), 80.44$ (CH-4'), 86.42 (CH-1'), 102.32 (Bu-C $\equiv \mathrm{C}-\mathrm{Pur}$ ), 134.89 (C-5), 143.03 (C-6), 143.18 (CH-8), 150.91 (C-4) 152.82 (CH-2), 169.29, 169.52 and 170.24 $(\mathrm{CO}) ; \mathrm{IR}\left(\mathrm{CHCl}_{3}\right) \vee 2999,2962,2232,1751,1585,1374,1245,1235,1048 \mathrm{~cm}^{-1}$; MS (FAB, 
m/z (rel.\%)) $459\left(\mathrm{M}^{+}+\mathrm{H}, 22\right), 259$ (21), 201 (100), 139 (85), 97 (76); HR-MS (FAB) calcd for $\mathrm{C}_{22} \mathrm{H}_{27} \mathrm{~N}_{4} \mathrm{O}_{7}\left[\mathrm{M}^{+}+\mathrm{H}\right] 459.1880$, found 459.1899. $\mathrm{R}_{f}($ hexane/EtOAc $1 / 3)=0.44$.

6-[(Trimethylsilyl)ethynyl]-9-(2,3,5-tri-O-acetyl- $\beta$-D-ribofuranosyl)-9H-purine (1c).

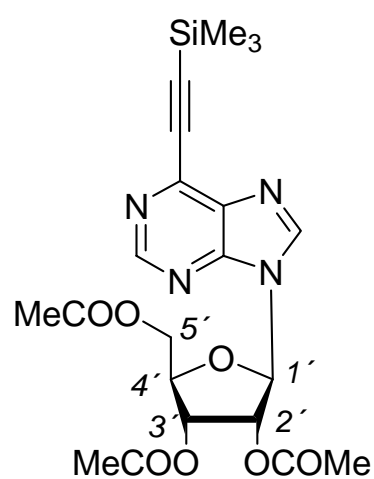

Column chromatography on silica gel (hexane/EtOAc 1/1) afforded $3.16 \mathrm{~g}(74 \%)$ of a yellowish oil, which after co-evaporation with diethyl ether formed a white foam. Experimental data were in accordance with the published data. ${ }^{5}$ 


\section{Synthesis of Protected 9-( $\beta$-D-Ribofuranosyl)-6-arylpurines.}

General procedure for $\mathrm{Ni}(\mathrm{cod})_{2} / 2 \mathrm{PPh}_{3}$ catalyzed cyclotrimerization of 6-alkynylpurines 1 with diynes 2. Method A.

Into a mixture of $\mathrm{Ni}(\operatorname{cod})_{2}(0.04 \mathrm{mmol}, 11 \mathrm{mg})$ and $\mathrm{PPh}_{3}(0.10 \mathrm{mmol}, 26 \mathrm{mg})$ was added a solution of 6-alkynylpurine $1(0.20 \mathrm{mmol})$ and diyne $2(0.22 \mathrm{mmol})$ in dry $\mathrm{MeCN}(4 \mathrm{~mL})$. The reaction mixture was initially stirred at $20^{\circ} \mathrm{C}$ for $24 \mathrm{~h}$ or until the consumption of the starting material (TLC). Then the solvent was evaporated under the reduced pressure and residue was subjected to column chromatography.

General procedure for $\mathrm{NiBr}_{2}(\mathrm{dppe}) / \mathrm{Zn}$ catalyzed cyclotrimerization of 6-alkynylpurines 1 with diynes 2 . Method $B$.

Into a solution of 6-alkynylpurine $1(0.20 \mathrm{mmol})$, diyne $2(0.22 \mathrm{mmol})$ and $\mathrm{NiBr}_{2}$ (dppe) $(0.04$ mmol, $25 \mathrm{mg}$ ) in dry $\mathrm{MeCN}(4 \mathrm{~mL})$ under Ar was added $\mathrm{Zn}$ powder $(10 \mathrm{mg}, 0.16 \mathrm{mmol})$. The reaction mixture was initially stirred at $20^{\circ} \mathrm{C}$ for $24 \mathrm{~h}$ or until the consumption of the starting material (TLC). Then the solvent was evaporated under the reduced pressure and residue was subjected to column chromatography.

General procedure for $\mathrm{CoBr}\left(\mathrm{PPh}_{3}\right)_{3}$ catalyzed cyclotrimerization of 6-alkynylpurines 1 with diynes 2 . Method $\mathrm{C}$.

Into a solution of 6-alkynylpurine $\mathbf{1}(0.20 \mathrm{mmol})$, diyne $\mathbf{2}(0.22 \mathrm{mmol})$ in dry toluene $(4 \mathrm{~mL})$ under $\mathrm{Ar}$ was added $\mathrm{CoBr}\left(\mathrm{PPh}_{3}\right)_{3}(0.04 \mathrm{mmol}, 37 \mathrm{mg})$. The reaction mixture was initially stirred at $20^{\circ} \mathrm{C}$ for $24 \mathrm{~h}$ or until the consumption of the starting material (TLC). Then the solvent was evaporated under the reduced pressure and residue was subjected to column chromatography.

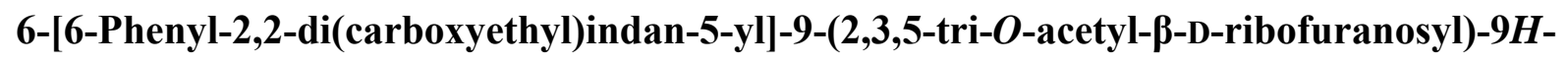
purine (3aa). (Method A)

Column chromatography on silica gel (diethyl ether) afforded $116 \mathrm{mg}(81 \%)$ of a white foam: $[\alpha]_{\mathrm{D}}-22.8\left(c\right.$ 0.23, $\left.\mathrm{CHCl}_{3}\right) ;{ }^{1} \mathrm{H} \mathrm{NMR}\left(500 \mathrm{MHz}, \mathrm{CDCl}_{3}\right) \delta 1.28\left(\mathrm{t}, 6 \mathrm{H}, J_{\text {vic }}=7.1, \mathrm{CH}_{3} \mathrm{CH}_{2}\right)$, 2.09, 2.10 and $2.15\left(3 \times \mathrm{s}, 3 \times 3 \mathrm{H}, \mathrm{CH}_{3} \mathrm{CO}\right), 3.70$ and $3.72\left(2 \times \mathrm{s}, 2 \times 2 \mathrm{H}, \mathrm{H}-7^{\prime \prime}\right.$ and $\left.\mathrm{H}-9^{\prime \prime}\right), 4.23(\mathrm{q}$, $\left.4 \mathrm{H}, J_{\text {vic }}=7.1, \mathrm{CH}_{2} \mathrm{CH}_{3}\right), 4.36\left(\mathrm{dd}, 1 \mathrm{H}, J_{\text {gem }}=13.1, J_{5^{\prime} \mathrm{b}, 4^{\prime}}=5.3, \mathrm{H}-5^{\prime} \mathrm{b}\right), 4.43-4.49$ (m, $2 \mathrm{H}, \mathrm{H}-$ $5^{\prime} \mathrm{a}$ and H-4' $), 5.67\left(\mathrm{t}, 1 \mathrm{H}, J_{3^{\prime}, 2^{\prime}}=5.3, J_{3^{\prime}, 4^{\prime}}=4.9, \mathrm{H}-3^{\prime}\right), 5.90\left(\mathrm{t}, 1 \mathrm{H}, J_{2^{\prime}, 3^{\prime}}=5.3, J_{2^{\prime}, 1^{\prime}}=5.0, \mathrm{H}-\right.$ $\left.2^{\prime}\right), 6.19\left(\mathrm{~d}, 1 \mathrm{H}, J_{1^{\prime}, 2^{\prime}}=5.0, \mathrm{H}-1^{\prime}\right), 7.12(\mathrm{~m}, 5 \mathrm{H}, \mathrm{Ph}), 7.38$ (s, 1H, H-3"), 7.60 (s, 1H, H-6"), 
8.03 (s, $1 \mathrm{H}, \mathrm{H}-8), 8.83$ (s, $1 \mathrm{H}, \mathrm{H}-2) ;{ }^{13} \mathrm{C}$ NMR $\left(125.8 \mathrm{MHz}, \mathrm{CDCl}_{3}\right)$

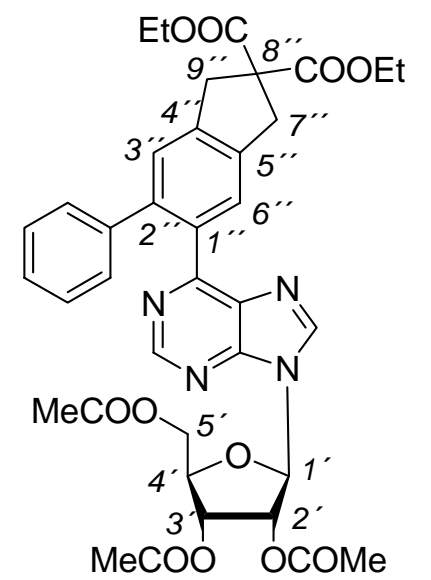

$\delta 14.02\left(\mathrm{CH}_{3} \mathrm{CH}_{2}\right), 20.38,20.51$ and $20.74\left(\mathrm{CH}_{3} \mathrm{CO}\right) ; 40.19$ and $40.43\left(\mathrm{CH}_{2}-7^{\prime \prime}\right.$ and $\left.\mathrm{CH}_{2}-9^{\prime \prime}\right) ; 60.60\left(\mathrm{C}-8^{\prime \prime}\right) ; 61.82\left(\mathrm{CH}_{2} \mathrm{CH}_{3}\right), 62.93$ $\left(\mathrm{CH}_{2}-5^{\prime}\right), 70.46\left(\mathrm{CH}-3^{\prime}\right), 73.11\left(\mathrm{CH}-2^{\prime}\right), 80.23\left(\mathrm{CH}-4^{\prime}\right), 86.44(\mathrm{CH}-$ $\left.1^{\prime}\right), 126.45(\mathrm{CH}-p-\mathrm{Ph}), 126.66$ and $126.72\left(\mathrm{CH}-3^{\prime \prime}\right.$ and $\left.\mathrm{CH}-6^{\prime \prime}\right)$, 127.82 (CH-o-Ph), 129.21 (CH-m-Ph), 132.81 (C-5), 133.05 (C-1"), 139.35 (C-5"), 141.19 and 141.21 (C-i-Ph and C-2"), 142.37 (CH-8), 142.45 (C-4"), 150.92 (C-4), 152.44 (CH-2), 159.77 (C-6), 169.27,

169.53 and $170.28\left(\mathrm{COCH}_{3}\right), 172.42(\mathrm{COOEt}) ; \mathrm{IR}\left(\mathrm{CHCl}_{3}\right) \vee 3031$, 2986, 1750, 1732, 1588, 1369, 1248, 1097, 1069, $1052 \mathrm{~cm}^{-1}$; MS (FAB, m/z (rel.\%) 715 $\left(\mathrm{M}^{+}+\mathrm{H}, 5\right), 457$ (15), 139 (76), 97 (100); HR-MS (FAB) calcd for $\mathrm{C}_{37} \mathrm{H}_{38} \mathrm{~N}_{4} \mathrm{O}_{11}\left[\mathrm{M}^{+}+\mathrm{H}\right]$ 715.2615, found 715.2632. EA calcd for $\mathrm{C}_{37} \mathrm{H}_{38} \mathrm{~N}_{4} \mathrm{O}_{11}$ : C 62.18, H 5.36, N 7.84; found: $\mathrm{C}$ $61.88, \mathrm{H} 5.34, \mathrm{~N} 7.58$. $\mathrm{R}_{f}(1 / 4$ hexane/EtOAc $)=0.45$. (Method B, yield $37 \%$. $)$

\section{6-[6-Phenyl-(2,2-diacetylindan-5-yl)]-9-(2,3,5-tri-O-acetyl- $\beta$-D-ribofuranosyl)-9H-purine} (3ab). (Method A)

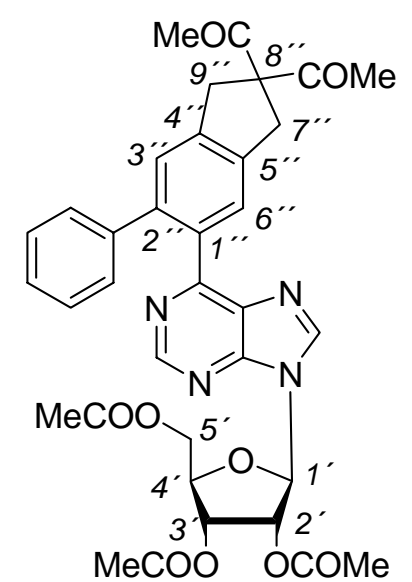

Column chromatography on silica gel (15/1 diethyl ether/acetone) afforded $113 \mathrm{mg}(86 \%)$ of a white foam: $[\alpha]_{\mathrm{D}}-23.3\left(\right.$ c $\left.0.24, \mathrm{CHCl}_{3}\right)$; ${ }^{1} \mathrm{H}$ NMR $\left(400 \mathrm{MHz}, \mathrm{CDCl}_{3}\right) \delta 2.09,2.10$ and $2.15(3 \times \mathrm{s}, 3 \times 3 \mathrm{H}$, $\left.\mathrm{CH}_{3} \mathrm{COO}\right), 2.22\left(\mathrm{~s}, 3 \mathrm{H}, \mathrm{CH}_{3} \mathrm{CO}\right), 3.63$ and $3.64\left(2 \times \mathrm{s}, 2 \times 2 \mathrm{H}, \mathrm{H}-7^{\prime \prime}\right.$ and $\left.\mathrm{H}-9^{\prime \prime}\right), 4.35\left(\mathrm{dd}, 1 \mathrm{H}, J_{\mathrm{gem}}=13.0, J_{5^{\prime} \mathrm{b}, 4^{\prime}}=5.3, \mathrm{H}-5^{\prime} \mathrm{b}\right), 4.42-4.49$ (m, 2H, H-5'a and H-4'), $5.67\left(\mathrm{t}, 1 \mathrm{H}, J_{3^{\prime}, 2^{\prime}}=5.5, J_{3^{\prime}, 4^{\prime}}=5.1, \mathrm{H}-3^{\prime}\right)$, $5.90\left(\mathrm{t}, 1 \mathrm{H}, J_{2^{\prime}, 3^{\prime}}=5.5, J_{2^{\prime}, 1^{\prime}}=5.0, \mathrm{H}-2^{\prime}\right), 6.18\left(\mathrm{~d}, 1 \mathrm{H}, J_{1^{\prime}, 2^{\prime}}=5.0, \mathrm{H}-\right.$ 1'), 7.08-7.16 (m, 5H, Ph), 7.39 (s, 1H, H-3"), 7.61 (s, 1H, H-6"), 8.03 (s, 1H, H-8), 8.83 (s, 1H, H-2); ${ }^{13} \mathrm{C} \mathrm{NMR} \mathrm{(125.8} \mathrm{MHz,} \mathrm{CDCl}_{3}$ ) $\delta 20.39,20.51$ and $20.74\left(\mathrm{CH}_{3} \mathrm{COO}\right), 26.59\left(\mathrm{CH}_{3} \mathrm{CO}\right), 37.37$ and $37.58\left(\mathrm{CH}_{2}-7^{\prime \prime}\right.$ and $\left.\mathrm{CH}_{2}-9^{\prime \prime}\right)$, $62.92\left(\mathrm{CH}_{2}-5^{\prime}\right), 70.45\left(\mathrm{CH}-3^{\prime}\right), 73.13\left(\mathrm{CH}-2^{\prime}\right), 74.99$ (C-8'), $80.23\left(\mathrm{CH}-4^{\prime}\right), 86.52\left(\mathrm{CH}-1^{\prime}\right)$, 126.56 (CH-p-Ph), 126.88 and 126.97 (CH-3" and CH-6"), 127.88 (CH-o-Ph), 129.18 (CH-mPh), 132.82 (C-5), 133.30 (C-1"), 138.99 (C-5"), 141.03 (C-i-Ph), 141.45 (C-2"), 142.12 (C4"), 142.46 (CH-8), 150.94 (C-4), 152.46 (CH-2), 159.57 (C-6), 169.28, 169.53 and 170.27 $\left(\mathrm{CH}_{3} \mathbf{C O O}\right), 204.53\left(\mathrm{COCH}_{3}\right) ;$ IR $\left(\mathrm{CHCl}_{3}\right) \vee 3028,2993,1751,1701,1588,1373,1243$, 1232, 1099, 1072, $1048 \mathrm{~cm}^{-1}$; MS (EI, m/z (rel.\%)) 654 (M+, 3), 611 (12), 395 (10), 353 (32), 
311 (10), 139 (15), 97 (35), 43 (100); HR-MS (EI) calcd for $\mathrm{C}_{35} \mathrm{H}_{34} \mathrm{~N}_{4} \mathrm{O}_{9}\left[\mathrm{M}^{+}\right]$654.2326, found 654.2354. $\mathrm{R}_{f}($ EtOAc $)=0.38$. (Method B, yield 62\%.)

6-[6-Phenyl-2-acetyl-2-(carboxyethyl)indan-5-yl]-9-(2,3,5-tri-O-acetyl- $\beta$-D-ribofuranosyl) -9H-purine (3ac). (Method A)

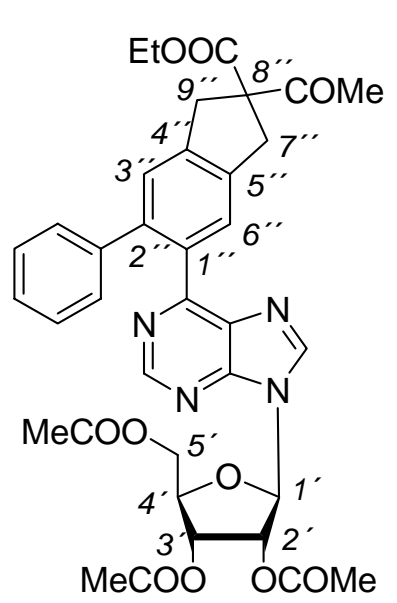

Column chromatography on silica gel (diethyl ether) afforded 115 mg $(84 \%)$ of a white foam: $[\alpha]_{\mathrm{D}}-29.0\left(\right.$ c $\left.0.24, \mathrm{CHCl}_{3}\right) ;{ }^{1} \mathrm{H}$ NMR $\left(500 \mathrm{MHz}, \mathrm{CDCl}_{3}\right) \delta 1.29\left(\mathrm{t}, 3 \mathrm{H}, J_{\text {vic }}=7.1, \mathrm{CH}_{3}-\mathrm{Et}\right), 2.09,2.10$ and $2.15\left(3 \times \mathrm{s}, 3 \times 3 \mathrm{H}, \mathrm{CH}_{3}-\mathrm{OAc}\right), 2.27\left(\mathrm{~s}, 3 \mathrm{H}, \mathrm{CH}_{3}-\mathrm{Ac}\right), 3.61,3.62,3.66$ and $3.69\left(4 \times \mathrm{d}, 4 \times 1 \mathrm{H}, J_{\text {gem }}=16.7, \mathrm{H}-7^{\prime \prime}\right.$ and $\left.\mathrm{H}-9{ }^{\prime \prime}\right), 4.25\left(\mathrm{q}, 2 \mathrm{H}, J_{\text {vic }}=\right.$ 7.1, $\left.\mathrm{CH}_{2}-\mathrm{Et}\right), 4.35$ (dd, $\left.1 \mathrm{H}, J_{\text {gem }}=13.1, J_{5^{\prime} \mathrm{b} 4^{\prime}}=5.3, \mathrm{H}^{\prime} 5^{\prime} \mathrm{b}\right), 4.43-4.48$ (m, 2H, H-5'a and H-4'), $5.67\left(\mathrm{t}, 1 \mathrm{H}, J_{3^{\prime} 2^{\prime}}=5.3, J_{3^{\prime} 4^{\prime}}=5.1, \mathrm{H}-3^{\prime}\right)$, $5.91\left(\mathrm{t}, 1 \mathrm{H}, J_{2^{\prime} 3^{\prime}}=5.3, J_{2^{\prime} 1^{\prime}}=5.0, \mathrm{H}-2^{\prime}\right), 6.19\left(\mathrm{~d}, 1 \mathrm{H}, J_{1^{\prime} 2^{\prime}}=5.0, \mathrm{H}-\right.$ $\left.1^{\prime}\right), 7.12$ (m, 5H, Ph), 7.38 (s, 1H, H-3"), 7.60 (s, 1H, H-6"), 8.03 (s, 1H, H-8), 8.82 (s, 1H, H-2); ${ }^{13} \mathrm{C}$ NMR (125.8 MHz, $\left.\mathrm{CDCl}_{3}\right) \delta 14.02\left(\mathrm{CH}_{3}-\mathrm{Et}\right), 20.38,20.51$ and $20.74\left(\mathrm{CH}_{3}-\mathrm{OAc}\right), 26.15\left(\mathrm{CH}_{3}-\mathrm{Ac}\right), 38.71$ and $38.92\left(\mathrm{CH}_{2}-7^{\prime \prime}\right.$ and $\left.\mathrm{CH}_{2}-9^{\prime \prime}\right), 61.96\left(\mathrm{CH}_{2}-\right.$ Et), $62.92\left(\mathrm{CH}_{2}-5^{\prime}\right), 67.12$ (C-8'), 70.44 (CH-3'), 73.11 (CH-2'), 80.21 (CH-4'), 86.46 (CH1'), 126.48 (CH-p-Ph), 126.78 and 126.82 (CH-3" and $\mathrm{CH}-6 "), 127.83$ (CH-o-Ph), 129.19 (CH-m-Ph), 132.81 (C-5), 133.12 (C-1"), 139.14 (C-5"), 141.13 (C-i-Ph), 141.29 (C-2"), 142.29 (C-4"), 142.40 (CH-8), 150.91 (C-4), 152.43 (CH-2), 159.68 (C-6), 169.28, 169.53 and $170.27(\mathrm{C}=\mathrm{O}$ from OAc $), 172.12(\mathrm{C}=\mathrm{O}$ form $\mathrm{COOEt}), 202.46(\mathrm{C}=\mathrm{O}$ form $\mathrm{Ac}) ; \quad \mathrm{IR}$ $\left(\mathrm{CHCl}_{3}\right) \vee 3030,2988,1750,1714,1588,1370,1235,1207,1097,1073,1048 \mathrm{~cm}^{-1}$; MS (EI, m/z (rel.\%)) $684\left(\mathrm{M}^{+}, 6\right), 425$ (17), 139 (20), 97 (35), 57 (30), 43 (100); HR-MS (EI) calcd for $\mathrm{C}_{36} \mathrm{H}_{36} \mathrm{~N}_{4} \mathrm{O}_{10}\left[\mathrm{M}^{+}\right]$684.2431, found 684.2407. EA calcd for $\mathrm{C}_{36} \mathrm{H}_{36} \mathrm{~N}_{4} \mathrm{O}_{10}$ : C 63.15, H 5.30, N 8.18; found: C 63.03, H 5.23, N 7.92. $\mathrm{R}_{f}($ EtOAc $)=0.49$. (Method B, yield 48\%.)

\section{6-[6-Phenyl-2-cyano-2-(carboxyethyl)indan-5-yl]-9-(2,3,5-tri-O-acetyl- $\beta$-D-ribofuranosyl)} -9H-purine (3ad). (Method A)

Column chromatography on silica gel (diethyl ether) afforded $114 \mathrm{mg}(85 \%)$ of a white foam: $[\alpha]_{\mathrm{D}}-29.2\left(c \mathrm{c} 0.25, \mathrm{CHCl}_{3}\right),{ }^{1} \mathrm{H} \mathrm{NMR}\left(500 \mathrm{MHz}, \mathrm{CDCl}_{3}\right) \delta 1.37\left(\mathrm{t}, 3 \mathrm{H}, J_{\mathrm{vic}}=7.1, \mathrm{CH}_{3} \mathrm{CH}_{2}\right)$, 2.09, 2.10 and $2.15\left(3 \times \mathrm{s}, 3 \times 3 \mathrm{H}, \mathrm{CH}_{3} \mathrm{CO}\right), 3.69,3.71,3.82$ and $3.84\left(4 \times \mathrm{d}, 4 \times 1 \mathrm{H}, J_{\mathrm{gem}}=16.6\right.$, H-7" and H-9"), 4.33 (q, 2H, $\left.J_{\text {vic }}=7.1, \mathrm{CH}_{2} \mathrm{CH}_{3}\right), 4.36\left(\mathrm{dd}, 1 \mathrm{H}, J_{\text {gem }}=13.1, J_{5^{\prime} \mathrm{b}, 4^{\prime}}=5.3\right.$, H-5'b), 4.44-4.49 (m, 2H, H-5'a and H-4' $), 5.68$ (t, 1H, $\left.J_{3^{\prime}, 2^{\prime}}=5.5, J_{3^{\prime}, 4^{\prime}}=5.0, \mathrm{H}-3^{\prime}\right), 5.91$ (t, 


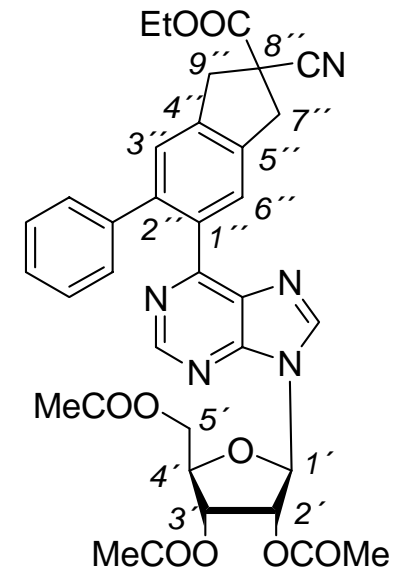

$\left.1 \mathrm{H}, J_{2^{\prime}, 3^{\prime}}=5.5, J_{2^{\prime}, 1^{\prime}}=4.9, \mathrm{H}-2^{\prime}\right), 6.19\left(\mathrm{~d}, 1 \mathrm{H}, J_{1^{\prime}, 2^{\prime}}=4.9, \mathrm{H}-1^{\prime}\right), 7.14$ (m, 5H, Ph), 7.43 (s, 1H, H-3"), 7.66 (s, 1H, H-6"), 8.05 (s, 1H, H-8), $8.83(\mathrm{~s}, 1 \mathrm{H}, \mathrm{H}-2) ; \quad{ }^{13} \mathrm{C}$ NMR $\left(125.8 \mathrm{MHz}, \mathrm{CDCl}_{3}\right) \quad \delta \quad 13.97$ $\left(\mathrm{CH}_{3} \mathrm{CH}_{2}\right), 20.39,20.51$ and $20.75\left(\mathrm{CH}_{3} \mathrm{CO}\right), 42.90$ and $42.97\left(\mathrm{CH}_{2}-\right.$ $7^{\prime \prime}$ and $\left.\mathrm{CH}_{2}-9^{\prime \prime}\right), 47.52\left(\mathrm{C}-8^{\prime \prime}\right), 62.92\left(\mathrm{CH}_{2}-5^{\prime}\right), 63.36\left(\mathrm{CH}_{2} \mathrm{CH}_{3}\right)$, 70.42 (CH-3'), 73.13 (CH-2'), 80.23 (CH-4'), 86.55 (CH-1' ), 120.34 (CN), 126.75 (CH-p-Ph), 126.89 and 126.99 (CH-3" and $\mathrm{CH}-6 ")$, 127.94 (CH-o-Ph), 129.16 (CH-m-Ph), 132.81 (C-5), 133.92 (C-1"), 137.39 (C-5"), 140.45 (C-i-Ph), 140.70 (C-2"), 142.14 (C-4"), 142.60 (CH-8), 151.00 (C-4), 152.45 (CH-2), 159.15 (C-6), 168.21 (COOEt), 169.30, 169.54 and $170.27\left(\mathrm{COCH}_{3}\right)$; IR $\left(\mathrm{CHCl}_{3}\right) \vee 3029,2989,2247,1749,1588,1371,1232,1098,1062,1050 \mathrm{~cm}^{-1} ; \mathrm{MS}(\mathrm{FAB}, \mathrm{m} / \mathrm{z}$ (rel.\%)) $668\left(\mathrm{M}^{+}+\mathrm{H}, 17\right), 410$ (78), 139(85), 97 (100); HR-MS (FAB) calcd for $\mathrm{C}_{35} \mathrm{H}_{34} \mathrm{~N}_{5} \mathrm{O}_{9}$ $\left[\mathrm{M}^{+}+\mathrm{H}\right]$ 668.2357, found 668.2346. EA calcd for $\mathrm{C}_{35} \mathrm{H}_{33} \mathrm{~N}_{5} \mathrm{O}_{9}$ : C 62.96, H 4.98, N 10.49; found: C 62.57, H 4.96, N 10.11. R $\mathrm{R}_{f}($ hexane/EtOAc 1/3)=0.47. (Method B, yield 35\%.)

6-[6-Phenyl-1,3-dihydro-2-tosyl-2H-indol-5-yl]-9-(2,3,5-tri-O-acetyl- $\beta$-D-ribofuranosyl)9H-purine (3ae). (Method A)

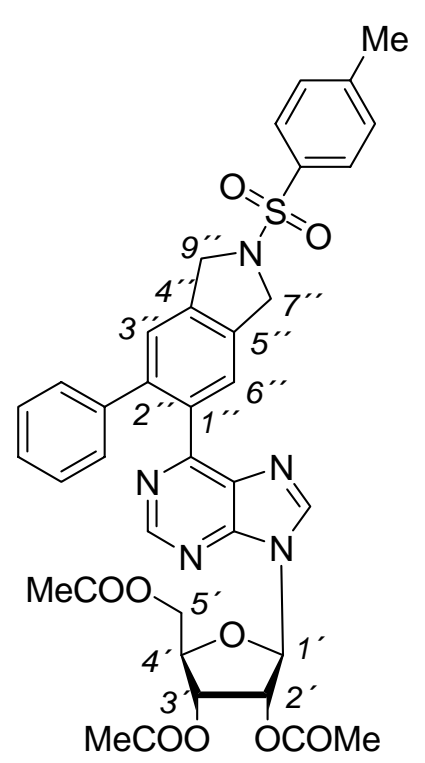

Column chromatography on silica gel (diethyl ether) afforded 70 $\mathrm{mg}(48 \%)$ of a white foam: $[\alpha]_{\mathrm{D}}-30.7\left(\right.$ c $\left.0.15, \mathrm{CHCl}_{3}\right) ;{ }^{1} \mathrm{H} \mathrm{NMR}$ $\left(400 \mathrm{MHz}, \mathrm{CDCl}_{3}\right) \delta 2.085,2.089$ and $2.147(3 \times \mathrm{s}, 3 \times 3 \mathrm{H}$, $\left.\mathrm{CH}_{3} \mathrm{CO}\right), 2.43\left(\mathrm{~s}, 3 \mathrm{H}, \mathrm{CH}_{3}\right), 4.35\left(\mathrm{dd}, 1 \mathrm{H}, J_{\text {gem }}=13.1, J_{5^{\prime} \mathrm{b}, 4^{\prime}}=5.3\right.$, H-5'b), 4.43-4.48 (m, 2H, H-5'a and H-4'), 4.74 (s, 4H, H-7"' and H-9"), 5.67 (t, 1H, $\left.J_{3^{\prime}, 2^{\prime}}=5.6, J_{3^{\prime}, 4^{\prime}}=4.8, \mathrm{H}-3^{\prime}\right), 5.89$ (t, 1H, $J_{2^{\prime}, 3^{\prime}}$ $\left.=5.6, J_{2^{\prime}, 1^{\prime}}=4.8, \mathrm{H}-2^{\prime}\right), 6.17\left(\mathrm{~d}, 1 \mathrm{H}, J_{1^{\prime}, 2^{\prime}}=4.8, \mathrm{H}-1^{\prime}\right)$, 7.05-7.15 (m, 5H, Ph), 7.33 (m, 2H, H-m-Tos), 7.34 (s, 1H, H-3"), 7.57 (s, 1H, H-6"), 7.79 (m, 2H, H-o-Tos), 8.03 (s, 1H, H-8), 8.80 (s, 1H, $\mathrm{H}-2) ;{ }^{13} \mathrm{C} \mathrm{NMR}\left(100.6 \mathrm{MHz}, \mathrm{CDCl}_{3}\right) \delta 20.38,20.49$ and 20.72 $\left(\mathrm{CH}_{3} \mathrm{CO}\right), 21.50\left(\mathrm{CH}_{3}\right), 53.62$ and $53.79\left(\mathrm{CH}_{2}-7^{\prime \prime}\right.$ and $\left.\mathrm{CH}_{2}-9^{\prime \prime}\right)$, $62.89\left(\mathrm{CH}_{2}-5^{\prime}\right), 70.40\left(\mathrm{CH}-3^{\prime}\right), 73.15\left(\mathrm{CH}-2^{\prime}\right), 80.22\left(\mathrm{CH}-4^{\prime}\right), 86.63\left(\mathrm{CH}-1^{\prime}\right), 125.01$ and 125.17 (CH-3" and CH-6"), 126.87 (CH-p-Ph), 127.55 (CH-o-Tos), 127.96 (CH-o-Ph), 129.09 (CH-m-Ph), 129.91 (CH-m-Tos), 132.83 (C-5), 133.50 (C-i-Tos), 133.95 (C-1"), 135.32 (C5"), 138.16 (C-4"), 140.43 (C-i-Ph), 142.13 (C-2"), 142.72 (CH-8), 143.85 (C-p-Tos), 150.99 (C-4), 152.42 (CH-2), 158.88 (C-6), 169.29, 169.52 and $170.23(\mathrm{CO}) ; \mathrm{IR}\left(\mathrm{CHCl}_{3}\right)$ v 3031, 
2994, 1751, 1588, 1374, 1348, 1333, 1247, 1164, 1098, 1066, $1049 \mathrm{~cm}^{-1} ; \mathrm{MS}(\mathrm{FAB}, \mathrm{m} / \mathrm{z}$ (rel.\%)) $726\left(\mathrm{M}^{+}+\mathrm{H}, 60\right), 468$ (88), 139 (100), 97 (92); HR-MS (FAB) calcd for $\mathrm{C}_{37} \mathrm{H}_{38} \mathrm{~N}_{4} \mathrm{O}_{11}$ $\left[\mathrm{M}^{+}+\mathrm{H}\right] 726.2234$, found $726.2200 . \mathrm{R}_{f}(1 / 3$ hexane/EtOAc $)=0.45$. (Method B, yield $70 \%$.)

6-[6-Phenyl-1,3-dihydro-2-phenyl-2H-indol-5-yl]-9-(2,3,5-tri-O-acetyl- $\beta$-D-ribofuranosyl) -9H-purine (3af). (Method A)

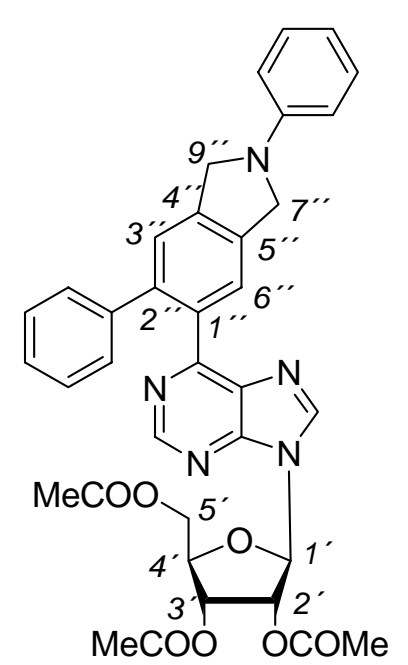

Column chromatography on silica gel (diethyl ether) afforded $47 \mathrm{mg}$ (36\%) of a white foam: $[\alpha]_{\mathrm{D}}-33.4\left(\right.$ c $\left.0.16, \mathrm{CHCl}_{3}\right) ;{ }^{1} \mathrm{H} \mathrm{NMR}(400$ $\left.\mathrm{MHz} \mathrm{CDCl}_{3}\right) \delta 2.10\left(\mathrm{~s}, 6 \mathrm{H}, \mathrm{CH}_{3} \mathrm{CO}\right), 2.16\left(\mathrm{~s}, 3 \mathrm{H}, \mathrm{CH}_{3} \mathrm{CO}\right), 4.36$ $\left(\mathrm{dd}, 1 \mathrm{H}, J_{\text {gem }}=13.0, J_{5^{\prime} \mathrm{b}, 4^{\prime}}=5.3, \mathrm{H}^{\prime} 5^{\prime} \mathrm{b}\right), 4.44-4.50\left(\mathrm{~m}, 2 \mathrm{H}, \mathrm{H}-5^{\prime} \mathrm{a}\right.$ and $\left.\mathrm{H}-4^{\prime}\right), 4.77$ (s, 4H, H-7" and H-9"), 5.69 (t, 1H, $J_{3^{\prime}, 2^{\prime}}=5.5, J_{3^{\prime}, 4^{\prime}}=$ 4.9, H-3'), $5.92\left(\mathrm{t}, 1 \mathrm{H}, J_{2^{\prime}, 3^{\prime}}=5.5, J_{2^{\prime}, 1^{\prime}}=4.9, \mathrm{H}-2^{\prime}\right), 6.20\left(\mathrm{~d}, 1 \mathrm{H}, J_{1^{\prime}, 2^{\prime}}\right.$ = 4.9, H-1'), 6.72 (m, 2H, H-o-Ph-8"), 6.78 (m, 1H, H-p-Ph-8"), 7.13-7.20 (m, 5H, Ph-2"), 7.33 (m, 2H, H-m-Ph-8"), 7.53 (s, 1H, H3"), 7.76 (s, 1H, H-6"), 8.06 (s, 1H, H-8), 8.86 (s, 1H, H-2); ${ }^{13} \mathrm{C}$ NMR (100.6 MHz, $\left.\mathrm{CDCl}_{3}\right) \delta 20.40,20.51$ and $20.75\left(\mathrm{CH}_{3} \mathrm{CO}\right)$, 53.52 and $53.71\left(\mathrm{CH}_{2}-7^{\prime \prime}\right.$ and $\left.\mathrm{CH}_{2}-9^{\prime \prime}\right), 62.92\left(\mathrm{CH}_{2}-5^{\prime}\right), 70.43\left(\mathrm{CH}-3^{\prime}\right), 73.15\left(\mathrm{CH}-2^{\prime}\right), 80.23$ (CH-4'), 86.57 (CH-1'), 111.62 (CH-o-Ph-8"), 116.33 (CH-p-Ph-8"), 125.06 (CH-3"), 125.21 (CH-6"), 126.68 (CH-p-Ph-2"), 127.95 (CH-m-Ph-2"), 129.25 (CH-o-Ph-2"), 129.42 (CH-mPh-8"), 132.91 (C-5), 133.36 (C-1"), 137.27 (C-5"), 140.19 (C-4"), 140.99 (C-i-Ph-2"), 141.53 (C-2"), 142.59 (CH-8), 147.01 (C-i-Ph-8"), 150.99 (C-4), 152.48 (CH-2), 159.50 (C-6), 169.31, 169.55 and 170.28 (CO); IR $\left(\mathrm{CHCl}_{3}\right) \vee 3030,1752,1589,1501,1377,1240,1098$, 1060, $1049 \mathrm{~cm}^{-1}$; MS (EI, m/z (rel.\%)) $647\left(\mathrm{M}^{+}, 16\right), 388$ (14), 43 (100); HR-MS (EI) calcd for $\mathrm{C}_{36} \mathrm{H}_{33} \mathrm{~N}_{5} \mathrm{O}_{7}\left[\mathrm{M}^{+}\right]$647.2380, found 647.2361. $\mathrm{R}_{f}($ hexane/EtOAc 1/1) $=0.35$. (Method B, yield 69\%. Method C, yield 30\%.)

\section{6-[6-Phenyl-1,3-dihydroisobenzofuran-5-yl)]-9-(2,3,5-tri-O-acetyl- $\beta$-D-ribofuranosyl)- 9H-purine (3ag). (Method A)}

Column chromatography on silica gel (diethyl ether/acetone 20/1) afforded $81 \mathrm{mg}(71 \%)$ of a white foam: $[\alpha]_{\mathrm{D}}-26.3\left(c \mathrm{c} 0.19, \mathrm{CHCl}_{3}\right) ;{ }^{1} \mathrm{H} \mathrm{NMR}\left(400 \mathrm{MHz}, \mathrm{CDCl}_{3}\right) \delta 2.10\left(\mathrm{~s}, 6 \mathrm{H}, \mathrm{CH}_{3} \mathrm{CO}\right)$, $2.15\left(\mathrm{~s}, 3 \mathrm{H}, \mathrm{CH}_{3} \mathrm{CO}\right), 4.36\left(\mathrm{dd}, 1 \mathrm{H}, J_{\mathrm{gem}}=13.0, J_{5^{\prime} \mathrm{b}, 4^{\prime}}=5.3, \mathrm{H}-5^{\prime} \mathrm{b}\right), 4.44-4.50$ (m, 2H, H-5'a and $\left.\mathrm{H}-4^{\prime}\right), 5.23$ (s, 4H, H-7" and H-9"), $5.68\left(\mathrm{t}, 1 \mathrm{H}, J_{3^{\prime}, 2^{\prime}}=5.5, J_{3^{\prime}, 4^{\prime}}=4.9, \mathrm{H}-3^{\prime}\right), 5.92(\mathrm{t}, 1 \mathrm{H}$, $\left.J_{2^{\prime}, 3^{\prime}}=5.5, J_{2^{\prime}, 1^{\prime}}=4.9, \mathrm{H}-2^{\prime}\right), 6.20\left(\mathrm{~d}, 1 \mathrm{H}, J_{1^{\prime}, 2^{\prime}}=4.9, \mathrm{H}-1^{\prime}\right), 7.14(\mathrm{~m}, 5 \mathrm{H}, \mathrm{Ph}), 7.42(\mathrm{~s}, 1 \mathrm{H}, \mathrm{H}-$ 


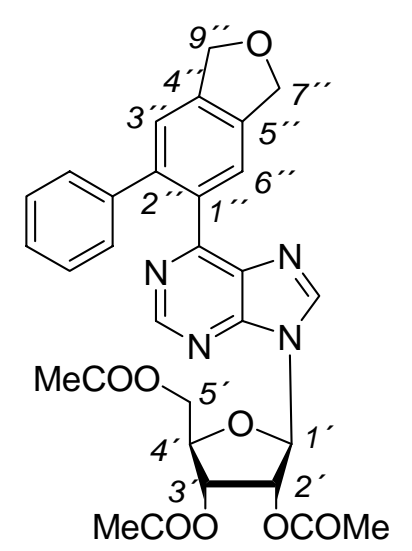

3"), 7.66 (s, 1H, H-6"), 8.06 (s, 1H, H-8), 8.84 (s, 1H, H-2; ${ }^{13} \mathrm{C}$ NMR $\left(100.6 \mathrm{MHz}, \mathrm{CDCl}_{3}\right) \delta 20.38,20.49$ and $20.72\left(\mathrm{CH}_{3} \mathrm{CO}\right), 62.90$ $\left(\mathrm{CH}_{2}-5^{\prime}\right), 70.43\left(\mathrm{CH}-3^{\prime}\right), 73.14\left(\mathrm{CH}-2^{\prime}\right), 73.39$ and $73.48\left(\mathrm{CH}_{2}-7^{\prime \prime}\right.$ and $\left.\mathrm{CH}_{2}-9^{\prime \prime}\right), 80.22\left(\mathrm{CH}-4^{\prime}\right), 86.57\left(\mathrm{CH}-1^{\prime}\right), 123.41\left(\mathrm{CH}-3^{\prime \prime}\right), 123.59$ (CH-6"), 126.68 (CH-p-Ph), 127.92 (CH-m-Ph), 129.22 (CH-o-Ph), 132.90 (C-5), 133.50 (C-1"), 138.40 (C-5"), 140.95 (C-i-Ph), 141.38 (C-2"), 141.73 (C-4"), 142.59 (CH-8), 150.99 (C-4), 152.44 (CH-2); 3010, 2995, 1751, 1589, 1371, 1247, 1098, $1048 \mathrm{~cm}^{-1}$; MS (FAB, m/z (rel.\%)) $573\left(\mathrm{M}^{+}+\mathrm{H}\right.$, 5), 315 (16), 279 (14), 55 (100); HR-MS (FAB) calcd for $\mathrm{C}_{30} \mathrm{H}_{29} \mathrm{~N}_{4} \mathrm{O}_{8}\left[\mathrm{M}^{+}+\mathrm{H}\right]$ 573.1985, found 573.1971. EA calcd for $\mathrm{C}_{30} \mathrm{H}_{28} \mathrm{~N}_{4} \mathrm{O}_{8}: \mathrm{C}$ 62.93, H 4.93, N 9.79; found: $\mathrm{C} 62.55, \mathrm{H}$ 5.00, N 9.10. $\mathrm{R}_{f}($ EtOAc $)=0.35$. (Method B, yield $45 \%$. Method C, yield $80 \%$.)

\section{6-[6-Phenyl-2-[[(1,1-dimethylethoxy)carbonyl]amino]-2-(carboxyethyl)indan-5-yl)]-9-}

\section{(2,3,5-tri-O-acetyl- $\beta$-D-ribofuranosyl)-9H-purine (3ah). (Method A)}

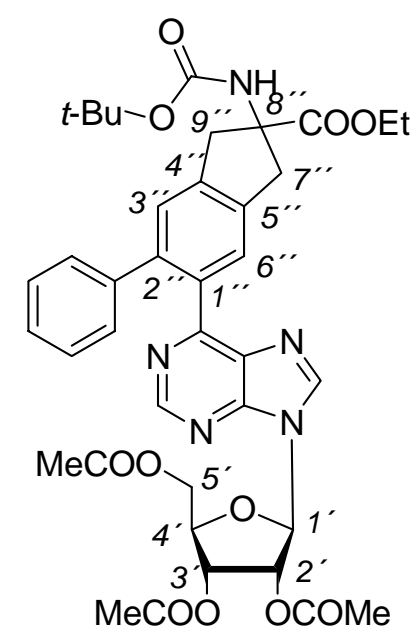

Column chromatography on silica gel (diethyl ether) afforded 133 $\mathrm{mg}(88 \%)$ of a white foam: $[\alpha]_{\mathrm{D}}-29.9\left(\right.$ c $\left.0.30, \mathrm{CHCl}_{3}\right) ;{ }^{1} \mathrm{H} \mathrm{NMR}$ $\left(400 \mathrm{MHz}, \mathrm{CDCl}_{3}\right) \delta 1.29\left(\mathrm{t}, 3 \mathrm{H}, J_{\text {vic }}=7.1, \mathrm{CH}_{3} \mathrm{CH}_{2}\right), 1.44(\mathrm{~s}, 9 \mathrm{H}$, $\left.\left(\mathrm{CH}_{3}\right)_{3} \mathrm{C}\right), 2.09,2.10$ and $2.15\left(3 \times \mathrm{s}, 3 \times 3 \mathrm{H}, \mathrm{CH}_{3} \mathrm{CO}\right), 3.31,3.33,3.71$ and $3.76\left(4 \times \mathrm{d}, 4 \mathrm{H}, J_{\mathrm{gem}}=16.6, \mathrm{H}-7^{\prime \prime}\right.$ and $\left.\mathrm{H}-9^{\prime \prime}\right), 4.26\left(\mathrm{q}, 2 \mathrm{H}, J_{\mathrm{vic}}=\right.$ 7.1, $\mathrm{CH}_{2}$-Et), $4.36\left(\mathrm{dd}, 1 \mathrm{H}, J_{\mathrm{gem}}=13.1, J_{5^{\prime} \mathrm{b}, 4^{\prime}}=5.3, \mathrm{H}-5^{\prime} \mathrm{b}\right), 4.43-$ 4.49 (m, 2H, H-5' a and H-4'), 5.15 (bs, 1H, NH), 5.68 (t, 1H, $J_{3^{\prime}, 2^{\prime}}=$ 5.4, $\left.J_{3^{\prime}, 4^{\prime}}=5.0, \mathrm{H}-3^{\prime}\right), 5.90$ and $5.91\left(2 \times \mathrm{t}, 1 \mathrm{H}, J_{2^{\prime}, 3^{\prime}}=5.4, J_{2^{\prime}, 1^{\prime}}=\right.$ 5.0, $\mathrm{H}-2^{\prime}$ diastereomeric), 6.19, $6.20\left(2 \times \mathrm{d}, 1 \mathrm{H}, J_{1^{\prime}, 2^{\prime}}=5.0, \mathrm{H}-1^{\prime}\right.$ diastereomeric), 7.08-7.16 (m, 5H, Ph), 7.39 (s, 1H, H-3"), $7.61(\mathrm{~s}$,

$\left.1 \mathrm{H}, \mathrm{H}-6^{\prime \prime}\right), 8.04,8.05\left(2 \times \mathrm{s}, 1 \mathrm{H}, \mathrm{H}-8\right.$ diastereomeric), $8.83(\mathrm{~s}, 1 \mathrm{H}, \mathrm{H}-2) ;{ }^{13} \mathrm{C} \mathrm{NMR}(100.6$ $\left.\mathrm{MHz}, \mathrm{CDCl}_{3}\right) \delta 14.15\left(\mathrm{CH}_{3} \mathrm{CH}_{2}\right), 20.38,20.51$ and $20.74\left(\mathrm{CH}_{3} \mathrm{CO}\right), 28.26\left(\left(\mathrm{CH}_{3}\right)_{3} \mathrm{C}\right), 43.50$ (br, $\mathrm{CH}_{2}-7^{\prime \prime}$ and $\left.\mathrm{CH}_{2}-9^{\prime \prime}\right), 61.65\left(\mathrm{CH}_{2} \mathrm{CH}_{3}\right), 62.92\left(\mathrm{CH}_{2}-5^{\prime}\right), 66.25\left(\mathrm{C}-8^{\prime \prime}\right), 70.45\left(\mathrm{CH}-3^{\prime}\right)$, $73.12\left(\mathrm{CH}-2^{\prime}\right), 80.23\left(\mathrm{CH}-4^{\prime}\right.$ and $\left.\mathbf{C}\left(\mathrm{CH}_{3}\right)_{3}\right), 86.47$ and 86.50 (CH-1' diastereomeric), 126.50 (CH-p-Ph), 127.05 (CH-3" and CH-6"), 127.86 (CH-o-Ph), 129.21 (CH-m-Ph), 132.83 (C-5), 133.10 (C-1"), 139.14 (C-5"), 141.14 (C-i-Ph), 141.31 (C-2"), 142.41 (C-4"), 142.46 (CH-8), 150.93 (C-4), 152.45 (CH-2), 154.90 (HNCOO), 159.72 (C-6), 169.29, 169.54 and 170.29 $\left(\mathrm{COCH}_{3}\right), 173.17$ (COOEt); IR $\left(\mathrm{CHCl}_{3}\right) \vee 3448,3029,2983,1749,1714,1588,1494,1369$, 
1247, 1161, 1063, $1050 \mathrm{~cm}^{-1}$; MS (FAB, m/z (rel.\%)) $758\left(\mathrm{M}^{+}+\mathrm{H}, 10\right), 444$ (110), 139 (43), 97 (42), 57 (100); HR-MS (FAB) calcd for $\mathrm{C}_{39} \mathrm{H}_{44} \mathrm{~N}_{5} \mathrm{O}_{11}\left[\mathrm{M}^{+}+\mathrm{H}\right]$ 758.3037, found 758.3046. $\mathrm{R}_{f}($ hexane/EtOAc $1 / 3)=0.59$.

6-[3-Phenyl-5,6,7,8-tetrahydronaphthalen-2-yl)]-9-(2,3,5-tri-O-acetyl- $\beta$-D-ribofuranosyl)9H-purine (3ai). (Method A)

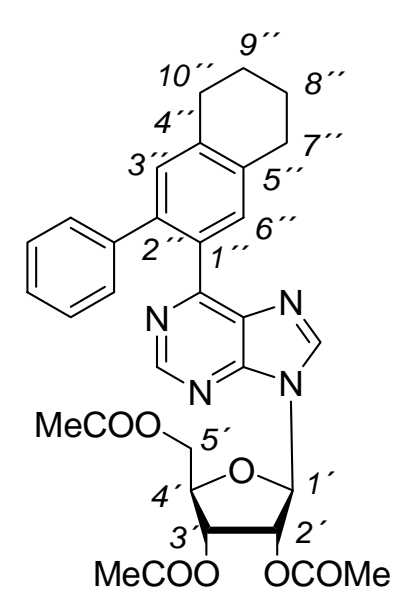

Column chromatography on silica gel (toluene/diethyl ether 1/1) afforded $15 \mathrm{mg}(13 \%)$ of a colourless oil: $[\alpha]_{\mathrm{D}}-73.7$ (c 0.07 , $\mathrm{CHCl}_{3}$ ); ${ }^{1} \mathrm{H} \mathrm{NMR}\left(400 \mathrm{MHz}, \mathrm{CDCl}_{3}\right) \delta 1.86$ (m, 4H, H-8",9"); 2.09 , 2.10 and $2.15\left(3 \times \mathrm{s}, 3 \times 3 \mathrm{H}, \mathrm{CH}_{3} \mathrm{CO}\right), 2.88\left(\mathrm{~m}, 4 \mathrm{H}, \mathrm{H}-7^{\prime \prime}, 10^{\prime \prime}\right), 4.36(\mathrm{~m}$, $\left.1 \mathrm{H}, \mathrm{H}-5^{\prime} \mathrm{b}\right), 4.43-4.49$ (m, 2H, H-5'a and H-4'), $5.68\left(\mathrm{dd}, 1 \mathrm{H}, J_{3^{\prime}, 2^{\prime}}=\right.$ $\left.5.5, J_{3^{\prime}, 4^{\prime}}=4.8, \mathrm{H}-3^{\prime}\right), 5.91\left(\mathrm{t}, 1 \mathrm{H}, J_{2^{\prime}, 3^{\prime}}=5.5, J_{2^{\prime}, 1^{\prime}}=5.1, \mathrm{H}-2^{\prime}\right), 6.20$ (d, $\left.1 \mathrm{H}, J_{1^{\prime}, 2^{\prime}}=5.1, \mathrm{H}-1^{\prime}\right), 7.09-7.15$ (m, 5H, Ph), 7.25 (s, 1H, H-3"), 7.48 (s, 1H, H-6"), 8.03 (s, 1H, H-8), 8.83 (s, 1H, H-2; ${ }^{13} \mathrm{C}$ NMR $\left(100.6 \mathrm{MHz}, \mathrm{CDCl}_{3}\right) \delta 20.39,20.51$ and $20.73\left(\mathbf{C H}_{3} \mathrm{CO}\right), 23.10$ and $23.15\left(\mathrm{CH}_{2}-8^{\prime \prime}, 9^{\prime \prime}\right), 29.05$ and $29.41\left(\mathrm{CH}_{2}-7^{\prime \prime}, 10^{\prime \prime}\right), 62.95\left(\mathrm{CH}_{2}-5^{\prime}\right), 70.49\left(\mathrm{CH}-3^{\prime}\right), 73.13(\mathrm{CH}-$ 2'), 80.23 (CH-4'), 86.41 (CH-1'), 126.24 (CH-p-Ph), 127.79 and 129.16 (CH-o,m-Ph); 131.19 (C-1"), 131.53 and 131.70 (CH-3" and CH-6"), 132.87 (C-5), 136.39 (C-5"), 139.00 (C-2"), 139.35 (C-4"), 141.27 (C-i-Ph), 142.25 (CH-8), 150.90 (C-4), 152.45 (CH-2), 159.92 (C-6), 169.29, 169.54 and 170.29 (CO); IR $\left(\mathrm{CHCl}_{3}\right) \vee 2929,1751,1588,1373,1237,1110$, 1075, $1047 \mathrm{~cm}^{-1}$; MS (FAB, m/z (rel.\%)) $585\left(\mathrm{M}^{+}+\mathrm{H}, 33\right), 327$ (100), 139 (43), 97 (43); HRMS (FAB) calcd for $\mathrm{C}_{32} \mathrm{H}_{33} \mathrm{~N}_{4} \mathrm{O}_{7}\left[\mathrm{M}^{+}+\mathrm{H}\right]$ 585.2349, found 585.2366. R $\mathrm{R}_{f}($ hexane/EtOAc 1/2) $=0.61$.

\section{6-[6-Butyl-2,2-di(carboxyethyl)indan-5-yl]-9-(2,3,5-tri-O-acetyl-ß-D-ribofuranosyl)-9H-} purine (3ba). (Method A)

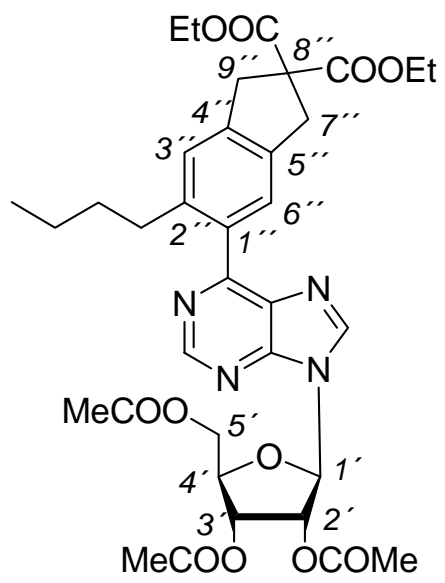

Column chromatography on silica gel (toluene/diethyl ether $1 / 1$ ) afforded $118 \mathrm{mg}(85 \%)$ of a colourless oil: $[\alpha]_{\mathrm{D}}-26.8$ (с 0.16 , $\left.\mathrm{CHCl}_{3}\right) ;{ }^{1} \mathrm{H} \mathrm{NMR}\left(400 \mathrm{MHz}, \mathrm{CDCl}_{3}\right) \delta 0.73\left(\mathrm{t}, 3 \mathrm{H}, J_{\text {vic }}=7.3\right.$, $\left.\mathrm{CH}_{3} \mathrm{CH}_{2} \mathrm{CH}_{2} \mathrm{CH}_{2}\right), 1.17\left(\mathrm{~m}, 2 \mathrm{H}, \mathrm{CH}_{3} \mathrm{CH}_{2} \mathrm{CH}_{2} \mathrm{CH}_{2}\right), 1.26(\mathrm{t}, 6 \mathrm{H}$, $\left.J_{\text {vic }}=7.1, \mathrm{CH}_{3} \mathrm{CH}_{2}\right), 1.42\left(\mathrm{~m}, 2 \mathrm{H}, \mathrm{CH}_{3} \mathrm{CH}_{2} \mathrm{CH}_{2} \mathrm{CH}_{2}\right), 2.11,2.13$ and $2.17\left(3 \times \mathrm{s}, 3 \times 3 \mathrm{H}, \mathrm{CH}_{3} \mathrm{CO}\right), 2.74\left(\mathrm{~m}, 2 \mathrm{H}, \mathrm{CH}_{3} \mathrm{CH}_{2} \mathrm{CH}_{2} \mathrm{CH}_{2}\right)$, 3.635 and $3.644\left(2 \times \mathrm{s}, 2 \times 2 \mathrm{H}, \mathrm{H}-7^{\prime \prime}\right.$ and H-9"), 4.21 (q, 4H, $J_{\text {vic }}=$ 
7.1, $\left.\mathrm{CH}_{2} \mathrm{CH}_{3}\right), 4.41\left(\mathrm{dd}, 1 \mathrm{H}, J_{\mathrm{gem}}=12.9, J_{5^{\prime} \mathrm{b}, 4^{\prime}}=5.2, \mathrm{H}-5^{\prime} \mathrm{b}\right), 4.47-4.52\left(\mathrm{~m}, 2 \mathrm{H}, \mathrm{H}-5^{\prime} \mathrm{a}\right.$ and H$\left.4^{\prime}\right), 5.73\left(\mathrm{t}, 1 \mathrm{H}, J_{3^{\prime}, 2^{\prime}}=5.5, J_{3^{\prime}, 4^{\prime}}=4.8, \mathrm{H}-3^{\prime}\right), 6.01\left(\mathrm{t}, 1 \mathrm{H}, J_{2^{\prime}, 3^{\prime}}=5.5, J_{2^{\prime}, 1^{\prime}}=5.1, \mathrm{H}-2^{\prime}\right), 6.29$ $\left(\mathrm{d}, 1 \mathrm{H}, J_{1^{\prime}, 2^{\prime}}=5.1, \mathrm{H}-1^{\prime}\right), 7.22$ (s, 1H, H-3"), 7.41 (s, 1H, H-6"), 8.21 (s, 1H, H-8), 9.03 (s, 1H, $\mathrm{H}-2) ;{ }^{13} \mathrm{C} \mathrm{NMR}\left(100.6 \mathrm{MHz}, \mathrm{CDCl}_{3}\right) \delta 13.74\left(\mathrm{CH}_{3} \mathrm{CH}_{2} \mathrm{CH}_{2} \mathrm{CH}_{2}\right), 14.01\left(\mathrm{CH}_{3} \mathrm{CH}_{2}\right), 20.41$, 20.53 and $20.75\left(\mathrm{CH}_{3} \mathrm{CO}\right), 22.42\left(\mathrm{CH}_{3} \mathrm{CH}_{2} \mathrm{CH}_{2} \mathrm{CH}_{2}\right), 32.89\left(\mathrm{CH}_{3} \mathrm{CH}_{2} \mathrm{CH}_{2} \mathrm{CH}_{2}\right), 33.59$ $\left(\mathrm{CH}_{3} \mathrm{CH}_{2} \mathrm{CH}_{2} \mathrm{CH}_{2}\right), 40.13$ and $40.46\left(\mathrm{CH}_{2}-7^{\prime \prime}\right.$ and $\left.\mathrm{CH}_{2}-9^{\prime \prime}\right), 60.53\left(\mathrm{C}-8^{\prime \prime}\right), 61.72\left(\mathrm{CH}_{2} \mathrm{CH}_{3}\right)$, $63.01\left(\mathrm{CH}_{2}-5^{\prime}\right), 70.54\left(\mathrm{CH}-3^{\prime}\right), 73.14\left(\mathrm{CH}-2^{\prime}\right), 80.33\left(\mathrm{CH}-4^{\prime}\right), 86.55\left(\mathrm{CH}-1^{\prime}\right), 125.83$ and 126.31 (CH-3" and CH-6"), 132.85 (C-5), 133.19 (C-1"), 137.57 (C-5"), 141.00 (C-2"), 142.17 (C-4"), 142.63 (CH-8), 151.17 (C-4), 152.50 (CH-2), 160.11 (C-6), 169.36, 169.57 and $170.30\left(\mathrm{COCH}_{3}\right), 171.57(\mathrm{COOEt}) ; \mathrm{IR}\left(\mathrm{CHCl}_{3}\right) \vee 3030,2985,1750,1731,1588,1369,1276$, 1245, 1097, 1068, $1052 \mathrm{~cm}^{-1}$; MS (FAB, m/z (rel.\%)) $695\left(\mathrm{M}^{+}+\mathrm{H}, 18\right), 437$ (55), 139 (100), 97 (100); HR-MS (FAB) calcd for $\mathrm{C}_{35} \mathrm{H}_{43} \mathrm{~N}_{4} \mathrm{O}_{11}\left[\mathrm{M}^{+}+\mathrm{H}\right]$ 695.2928, found 695.2935. $\mathrm{R}_{f}($ hexane/EtOAc $1 / 2)=0.66$. (Method B, yield 23\%.)

\section{6-[6-Butyl-2-cyano-2-(carboxyethyl)indan-5-yl]-9-(2,3,5-tri-O-acetyl- $\beta$-D-ribofuranosyl)-} 9H-purine (3bd). (Method A)

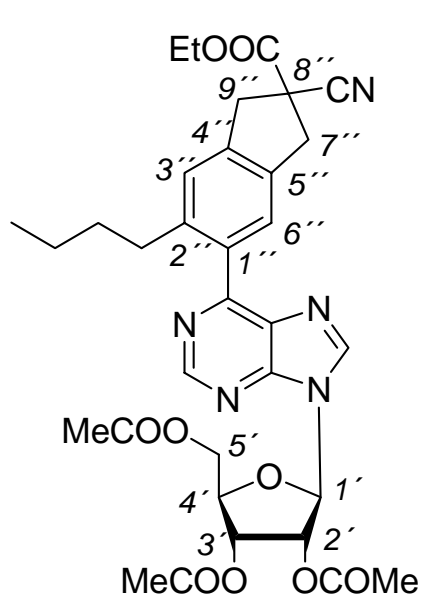

Column chromatography on silica gel (toluene/diethyl ether 1/1) afforded $50 \mathrm{mg}(39 \%)$ of a colourless oil: $[\alpha]_{\mathrm{D}}-17.5$ (c 0.17 , $\left.\mathrm{CHCl}_{3}\right) ; \quad{ }^{1} \mathrm{H} \mathrm{NMR}\left(500 \mathrm{MHz}, \mathrm{CDCl}_{3}\right) \delta 0.75\left(\mathrm{t}, 3 \mathrm{H}, J_{\text {vic }}=7.3\right.$, $\left.\mathrm{CH}_{3} \mathrm{CH}_{2} \mathrm{CH}_{2} \mathrm{CH}_{2}\right), 1.18\left(\mathrm{~m}, 2 \mathrm{H}, \mathrm{CH}_{3} \mathrm{CH}_{2} \mathrm{CH}_{2} \mathrm{CH}_{2}\right), 1.35$ (t, $3 \mathrm{H}$, $\left.J_{\text {vic }}=7.1, \mathrm{CH}_{3} \mathrm{CH}_{2}\right), 1.44\left(\mathrm{~m}, 2 \mathrm{H}, \mathrm{CH}_{3} \mathrm{CH}_{2} \mathrm{CH}_{2} \mathrm{CH}_{2}\right), 2.12,2.13$ and $2.17\left(3 \times \mathrm{s}, 3 \times 3 \mathrm{H}, \mathrm{CH}_{3} \mathrm{CO}\right), 2.76\left(\mathrm{~m}, 2 \mathrm{H}, \mathrm{CH}_{3} \mathrm{CH}_{2} \mathrm{CH}_{2} \mathrm{CH}_{2}\right)$, $3.61,3.63,3.74$ and $3.77\left(4 \times \mathrm{d}, 4 \times 1 \mathrm{H}, J_{\text {gem }}=16.6, \mathrm{H}-7^{\prime \prime}\right.$ and $\left.\mathrm{H}-9^{\prime \prime}\right)$, $4.31\left(\mathrm{q}, 2 \mathrm{H}, J_{\text {vic }}=7.1, \mathrm{CH}_{2} \mathrm{CH}_{3}\right), 4.41\left(\mathrm{dd}, 1 \mathrm{H}, J_{\text {gem }}=13.0, J_{5^{\prime} \mathrm{b}, 4^{\prime}}=\right.$ 5.2, H-5'b), 4.48-4.52 (m, 2H, H-5' a and H-4'), 5.73 (t, $1 \mathrm{H}, J_{3^{\prime}, 2^{\prime}}=$ 5.5, $\left.J_{3^{\prime}, 4^{\prime}}=4.9, \mathrm{H}-3^{\prime}\right), 6.01\left(\mathrm{t}, 1 \mathrm{H}, J_{2^{\prime}, 3^{\prime}}=5.5, J_{2^{\prime}, 1^{\prime}}=5.0, \mathrm{H}-2^{\prime}\right), 6.29\left(\mathrm{~d}, 1 \mathrm{H}, J_{1^{\prime}, 2^{\prime}}=5.0, \mathrm{H}-1^{\prime}\right)$, 7.27 (s, 1H, H-3"), 7.47 (s, 1H, H-6"), 8.23 (s, 1H, H-8), 9.04 (s, 1H, H-2); ${ }^{13} \mathrm{C}$ NMR (125.8 $\left.\mathrm{MHz}, \mathrm{CDCl}_{3}\right) \delta 13.72\left(\mathrm{CH}_{3} \mathrm{CH}_{2} \mathrm{CH}_{2} \mathrm{CH}_{2}\right), 13.95\left(\mathrm{CH}_{3} \mathrm{CH}_{2}\right), 20.41,20.53$ and 20.75 $\left(\mathbf{C H}_{3} \mathrm{CO}\right), 22.39\left(\mathrm{CH}_{3} \mathbf{C H}_{2} \mathrm{CH}_{2} \mathrm{CH}_{2}\right), 32.89\left(\mathrm{CH}_{3} \mathrm{CH}_{2} \mathrm{CH}_{2} \mathrm{CH}_{2}\right), 33.50\left(\mathrm{CH}_{3} \mathrm{CH}_{2} \mathbf{C H}_{2} \mathrm{CH}_{2}\right)$, 42.89 and $42.99\left(\mathrm{CH}_{2}-7^{\prime \prime}\right.$ and $\left.\mathrm{CH}_{2}-9^{\prime \prime}\right), 47.45\left(\mathrm{C}-8^{\prime \prime}\right), 62.98\left(\mathrm{CH}_{2}-5^{\prime}\right), 63.24\left(\mathrm{CH}_{2} \mathrm{CH}_{3}\right), 70.49$ (CH-3'), $73.16\left(\mathrm{CH}^{2}{ }^{\prime}\right), 80.31$ (CH-4'), $86.65\left(\mathrm{CH}-1^{\prime}\right), 120.52(\mathrm{CN}), 126.07$ (CH-3"), 126.59 (CH-6"), 132.83 (C-5), 134.06 (C-1"), 135.66 (C-5"), 140.19 (C-4"), 142.09 (C-2"), 142.87 (CH-8), 151.24 (C-4), 152.52 (CH-2), 159.46 (C-6), 168.36 (COOEt), 169.38, 169.58 and 
$170.29\left(\mathrm{COCH}_{3}\right)$; IR $\left(\mathrm{CHCl}_{3}\right)$ v 3029, 2989, 2246, 1749, 1588, 1371, 1232, 1097, $1064 \mathrm{~cm}^{-1}$; MS (FAB, m/z (rel.\%)) $648\left(\mathrm{M}^{+}+\mathrm{H}, 19\right), 390$ (74), 259 (24), 139 (100), 97 (76); HR-MS (FAB) calcd for $\mathrm{C}_{33} \mathrm{H}_{38} \mathrm{~N}_{5} \mathrm{O}_{9}\left[\mathrm{M}^{+}+\mathrm{H}\right]$ 648.2670, found 648.2648. $\mathrm{R}_{f}($ hexane/EtOAc $1 / 2)=$ 0.61 .

6-[6-Butyl-1,3-dihydroisobenzofuran-5-yl]-9-(2,3,5-tri-O-acetyl- $\beta$-D-ribofuranosyl)-9Hpurine (3bg). (Method A)

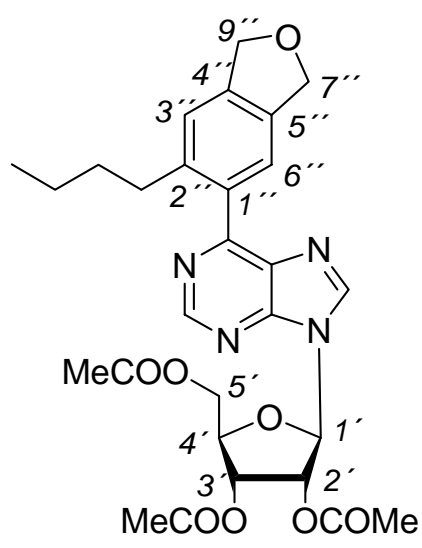

Column chromatography on silica gel (diethyl ether/acetone 12/1) afforded $24 \mathrm{mg}(22 \%)$ of a yellowish oil: $[\alpha]_{\mathrm{D}}-25.5$ (c 0.22 , $\left.\mathrm{CHCl}_{3}\right),{ }^{1} \mathrm{H} \mathrm{NMR}\left(400 \mathrm{MHz}, \mathrm{CDCl}_{3}\right) \delta 0.75\left(\mathrm{t}, 3 \mathrm{H}, J_{\text {vic }}=7.4\right.$, $\left.\mathrm{CH}_{3} \mathrm{CH}_{2} \mathrm{CH}_{2} \mathrm{CH}_{2}\right), 1.20$ and $1.45\left(2 \times \mathrm{m}, 2 \times 2 \mathrm{H}, \mathrm{CH}_{3} \mathrm{CH}_{2} \mathrm{CH}_{2} \mathrm{CH}_{2}\right)$, $2.12,2.13$ and $2.17\left(3 \times \mathrm{s}, 3 \times 3 \mathrm{H}, \quad \mathrm{CH}_{3} \mathrm{CO}\right), 2.79(\mathrm{~m}, 2 \mathrm{H}$, $\left.\mathrm{CH}_{3} \mathrm{CH}_{2} \mathrm{CH}_{2} \mathrm{CH}_{2}\right), 4.41\left(\mathrm{dd}, 1 \mathrm{H}, J_{\text {gem }}=13.0, J_{5^{\prime} \mathrm{b}, 4^{\prime}}=5.3, \mathrm{H}-5^{\prime} \mathrm{b}\right)$, 4.43-4.53 (m, 2H, H-5'a and H-4'), 5.16 (s, 4H, H-7" and H-9"),

$5.74\left(\mathrm{t}, 1 \mathrm{H}, J_{3^{\prime}, 2^{\prime}}=5.5, J_{3^{\prime}, 4^{\prime}}=4.9, \mathrm{H}-3^{\prime}\right), 6.02\left(\mathrm{t}, 1 \mathrm{H}, J_{2^{\prime}, 3^{\prime}}=5.5\right.$, $\left.J_{2^{\prime}, 1^{\prime}}=5.0, \mathrm{H}-2^{\prime}\right), 6.30\left(\mathrm{~d}, 1 \mathrm{H}, J_{1^{\prime}, 2^{\prime}}=5.0, \mathrm{H}-1^{\prime}\right), 7.27\left(\mathrm{~s}, 1 \mathrm{H}, \mathrm{H}-3^{\prime \prime}\right), 7.47$ (s, 1H, H-6"); 8.23 (s, 1H, H-8), 9.06 (s, 1H, H-2); ${ }^{13} \mathrm{C}$ NMR (100.6 MHz, $\left.\mathrm{CDCl}_{3}\right) \delta 13.73\left(\mathrm{CH}_{3} \mathrm{CH}_{2} \mathrm{CH}_{2} \mathrm{CH}_{2}\right)$, 20.41, 20.53 and $20.75\left(\mathbf{C H}_{3} \mathrm{CO}\right), 22.38\left(\mathrm{CH}_{3} \mathbf{C H}_{2} \mathrm{CH}_{2} \mathrm{CH}_{2}\right), 32.92\left(\mathrm{CH}_{3} \mathrm{CH}_{2} \mathrm{CH}_{2} \mathbf{C H}_{2}\right), 33.61$ $\left(\mathrm{CH}_{3} \mathrm{CH}_{2} \mathrm{CH}_{2} \mathrm{CH}_{2}\right), 62.99\left(\mathrm{CH}_{2}-5^{\prime}\right), 70.51\left(\mathrm{CH}-3^{\prime}\right), 73.17\left(\mathrm{CH}-2^{\prime}\right), 73.40$ and $73.48\left(\mathrm{CH}_{2}-7^{\prime \prime}\right.$ and $\left.\mathrm{CH}_{2}-9^{\prime \prime}\right), 80.32$ (CH-4'), 86.68 (CH-1'), 122.56 (CH-3"), 123.22 (CH-6"), 132.92 (C-5), 133.54 (C-1"), 136.75 (C-5"), 141.13 (C-4"), 141.59 (C-2"), 142.87 (CH-8), 151.24 (C-4), 152.52 (CH-2), 159.81 (C-6), 169.39, 169.57 and 170.29 (CO); IR $\left(\mathrm{CHCl}_{3}\right) \vee 3030,2960$, 1751, 1588, 1372, 1232, 1098, $1049 \mathrm{~cm}^{-1}$; MS (FAB, m/z (rel.\%)) $553\left(\mathrm{M}^{+}+\mathrm{H}, 30\right), 295$ (68), 259 (27), 139 (100), 97 (79); HR-MS (FAB) calcd for $\mathrm{C}_{28} \mathrm{H}_{33} \mathrm{~N}_{4} \mathrm{O}_{8}\left[\mathrm{M}^{+}+\mathrm{H}\right]$ 553.2298, found 553.2279. $\mathrm{R}_{f}($ EtOAc $)=0.60 .($ Method C , yield 28\%.

6-[6-Trimethylsilyl-2,2-di(carboxyethyl)indan-5-yl]-9-(2,3,5-tri-O-acetyl- $\beta$-D-ribofuranosyl)-9H-purine (3ca). (Method A)

Column chromatography on silica gel (toluene/diethyl ether 1/1) afforded $94 \mathrm{mg}$ (66\%) of a white foam: $[\alpha]_{\mathrm{D}}-21.7\left(\right.$ c $\left.0.28, \mathrm{CHCl}_{3}\right) ;{ }^{1} \mathrm{H} \mathrm{NMR}\left(400 \mathrm{MHz}, \mathrm{CDCl}_{3}\right) \delta 0.15(\mathrm{~s}, 9 \mathrm{H}$, $\left.\left(\mathrm{CH}_{3}\right)_{3} \mathrm{Si}\right), 1.27\left(\mathrm{t}, 6 \mathrm{H}, J_{\text {vic }}=7.1, \mathrm{CH}_{3} \mathrm{CH}_{2}\right), 2.11,2.13$ and $2.17\left(3 \times \mathrm{s}, 3 \times 3 \mathrm{H}, \mathrm{CH}_{3} \mathrm{CO}\right), 3.67$ and $3.72\left(2 \times \mathrm{s}, 2 \times 2 \mathrm{H}, \mathrm{H}-7 "\right.$ and $\left.\mathrm{H}-9{ }^{\prime \prime}\right), 4.22\left(\mathrm{q}, 4 \mathrm{H}, J_{\text {vic }}=7.1, \mathrm{CH}_{2} \mathrm{CH}_{3}\right), 4.41\left(\mathrm{dd}, 1 \mathrm{H}, J_{\text {gem }}=\right.$ $\left.12.9, J_{5^{\prime} \mathrm{b}, 4^{\prime}}=5.1, \mathrm{H}-5^{\prime} \mathrm{b}\right), 4.46-4.52\left(\mathrm{~m}, 2 \mathrm{H}, \mathrm{H}-5^{\prime} \mathrm{a}\right.$ and H-4'), $5.73\left(\mathrm{dd}, 1 \mathrm{H}, J_{3^{\prime}, 2^{\prime}}=5.6, J_{3^{\prime}, 4^{\prime}}=\right.$ 


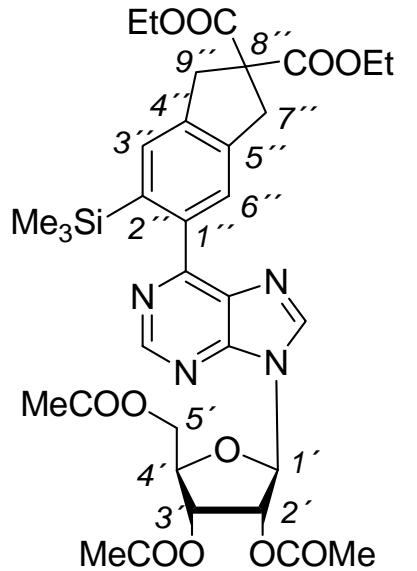

4.6, H-3'), $6.00\left(\mathrm{t}, 1 \mathrm{H}, J_{2^{\prime}, 3^{\prime}}=5.6, J_{2^{\prime}, 1^{\prime}}=5.2, \mathrm{H}-2^{\prime}\right), 6.31(\mathrm{~d}, 1 \mathrm{H}$, $\left.J_{1^{\prime}, 2^{\prime}}=5.2, \mathrm{H}-1^{\prime}\right), 7.65$ (s, 1H, H-3"), 8.22 (s, 1H, H-6"), $8.26(\mathrm{~s}, 1 \mathrm{H}$, $\mathrm{H}-8) ; 8.95$ (s, 1H, H-2); ${ }^{13} \mathrm{C}$ NMR (125.8 MHz, $\left.\mathrm{CDCl}_{3}\right) \delta 1.62$ $\left(\left(\mathrm{CH}_{3}\right)_{3} \mathrm{Si}\right), 14.01\left(\mathrm{CH}_{3} \mathrm{CH}_{2}\right), 20.39,20.53$ and $20.76\left(\mathrm{CH}_{3} \mathrm{CO}\right)$, 40.49 and $40.55\left(\mathrm{CH}_{2}-7^{\prime \prime}\right.$ and $\left.\mathrm{CH}_{2}-9^{\prime \prime}\right), 60.28$ (C-8"), 61.75 $\left(\mathrm{CH}_{2} \mathrm{CH}_{3}\right), 63.01\left(\mathrm{CH}_{2}-5^{\prime}\right), 70.57\left(\mathrm{CH}-3^{\prime}\right), 73.18\left(\mathrm{CH}-2^{\prime}\right), 80.34$ (CH-4'), 86.41 (CH-1'), 127.35 (CH-6"), 131.79 (C-5), 132.00 (CH-

3"), 139.96 and 140.09 (C-1" and C-2"), 140.89 (C-5"), 141.80 (C4"), 142.39 (CH-8), 151.54 (CH-2), 151.58 (C-4), 158.88 (C-6), 169.33, 169.55 and 170.29 $\left(\mathrm{COCH}_{3}\right), 172.42$ (COOEt); IR $\left(\mathrm{CHCl}_{3}\right) \vee 3030,2986,1750,1732,1585,1577,1369,1247$, 1193, 1069, $1052 \mathrm{~cm}^{-1}$; MS (FAB, m/z (rel.\%)) $711\left(\mathrm{M}^{+}+\mathrm{H}, 17\right), 437$ (13), 139 (7), 97 (12), 73 (100); HR-MS (FAB) calcd for $\mathrm{C}_{34} \mathrm{H}_{43} \mathrm{~N}_{4} \mathrm{O}_{11} \mathrm{Si}\left[\mathrm{M}^{+}+\mathrm{H}\right]$ 711.2698, found 711.2709. EA calcd for $\mathrm{C}_{34} \mathrm{H}_{42} \mathrm{~N}_{4} \mathrm{O}_{11} \mathrm{Si}$ : C 57.45, $\mathrm{H}$ 5.96, N 7.88; found: C 57.30, H 5.90, N 7.81. $\mathrm{R}_{f}($ hexane/EtOAc $1 / 2)=0.60$. (Method B, yield 18\%.)

6-[6-Trimethylsilyl-1,3-dihydro-2-tosyl-2H-indol-5-yl]-9-(2,3,5-tri-O-acetyl- $\beta$-D-ribofuranosyl)-9H-purine (3ce). (Method A)

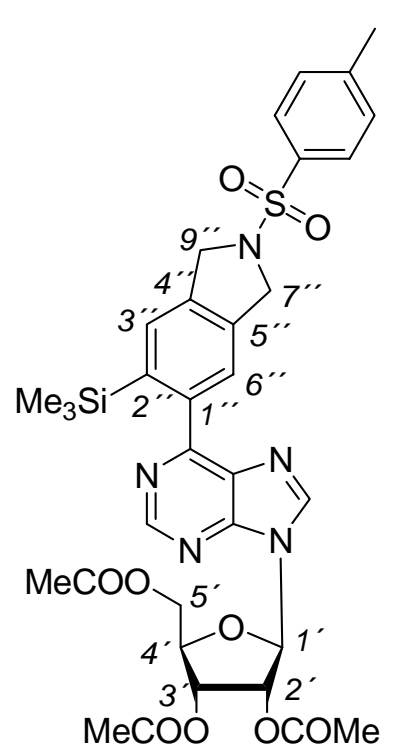

Column chromatography on silica gel (toluene/diethyl ether 1/1) afforded $33 \mathrm{mg}(23 \%)$ of a yellowish oil: $[\alpha]_{\mathrm{D}}-21.8\left(\right.$ c $\left.0.17, \mathrm{CHCl}_{3}\right)$; ${ }^{1} \mathrm{H}$ NMR $\left(400 \mathrm{MHz}, \mathrm{CDCl}_{3}\right) \delta 0.12\left(\mathrm{~s}, 9 \mathrm{H},\left(\mathrm{CH}_{3}\right)_{3} \mathrm{Si}\right), 2.10,2.12$ and $2.17\left(3 \times \mathrm{s}, 3 \times 3 \mathrm{H}, \mathrm{CH}_{3} \mathrm{CO}\right), 2.41$ (s, $\left.3 \mathrm{H}, \mathrm{CH}_{3}-\mathrm{Tos}\right), 4.40$ (dd, $1 \mathrm{H}, J_{\mathrm{gem}}$ $\left.=12.9, J_{5^{\prime} \mathrm{b}, 4^{\prime}}=5.2, \mathrm{H}-5^{\prime} \mathrm{b}\right), 4.46-4.52\left(\mathrm{~m}, 2 \mathrm{H}, \mathrm{H}-5^{\prime} \mathrm{a}\right.$ and H-4' $)$, 4.69$4.73\left(\mathrm{~m}, 4 \mathrm{H}, \mathrm{H}-7^{\prime \prime}\right.$ and H-9"), $5.72\left(\mathrm{dd}, 1 \mathrm{H}, J_{3^{\prime}, 2^{\prime}}=5.6, J_{3^{\prime}, 4^{\prime}}=4.7\right.$, H$\left.3^{\prime}\right), 5.98\left(\mathrm{t}, 1 \mathrm{H}, J_{2^{\prime}, 3^{\prime}}=5.6, J_{2^{\prime}, 1^{\prime}}=5.0, \mathrm{H}-2^{\prime}\right), 6.29\left(\mathrm{~d}, 1 \mathrm{H}, J_{1^{\prime}, 2^{\prime}}=5.0\right.$, H-1'), 7.32 (m, 2H, H-m-Tos), 7.61 (s, 1H, H-3"), 7.78 (m, 2H, H-oTos), 8.17 (s, 1H, H-6"), 8.24 (s, 1H, H-8), 8.95 (s, 1H, H-2); ${ }^{13} \mathrm{C}$ NMR (100.6 MHz, $\left.\mathrm{CDCl}_{3}\right) \delta 1.46\left(\left(\mathrm{CH}_{3}\right)_{3} \mathrm{Si}\right), 20.40,20.53$ and 20.76 $\left(\mathrm{CH}_{3} \mathrm{CO}\right), 21.48\left(\mathrm{CH}_{3}-\mathrm{Tos}\right), 53.82\left(\mathrm{CH}_{2}-7^{\prime \prime}\right.$ and $\left.\mathrm{CH}_{2}-9^{\prime \prime}\right), 62.97\left(\mathrm{CH}_{2}-\right.$ 5'), 70.50 (CH-3'), 73.21 (CH-2'), 80.33 (CH-4'), 86.62 (CH-1'), 125.57 (CH-6"), 127.60 (CH-o-Tos), 129.85 (CH-m-Tos), 130.25 (CH-3"), 131.86 (C-5), 133.50 (C-i-Tos), 136.92 (C5"), 137.50 (C-4"), 140.71 (C-1"), 141.38 (C-2"), 142.77 (CH-8), 143.75 (C-p-Tos), 151.60 (CH-2 and C-4), 158.16 (C-6), 169.37, 169.57 and 170.27 (CO); IR $\left(\mathrm{CHCl}_{3}\right) \vee 3031,3001$, 1751, 1586, 1374, 1348, 1331, 1230, 1164, 1099, 1066, $1049 \mathrm{~cm}^{-1} ;$ MS (FAB, m/z (rel.\%)) 
$722\left(\mathrm{M}^{+}+\mathrm{H}, 30\right), 448$ (17), 139 (70), 97 (71), 73 (100); HR-MS (FAB) calcd for $\mathrm{C}_{34} \mathrm{H}_{40} \mathrm{~N}_{5} \mathrm{O}_{9} \mathrm{SiS}\left[\mathrm{M}^{+}+\mathrm{H}\right]$ 722.2316, found 722.2329. $\mathrm{R}_{f}($ hexane/EtOAc 1/2) $=0.56$. (Method B, yield 41\%.)

6-[6-Trimethylsilyl-1,3-dihydroisobenzofuran-5-yl]-9-(2,3,5-tri-O-acetyl- $\beta$-D-ribofuranosyl)-9H-purine (3cg). (Method A)

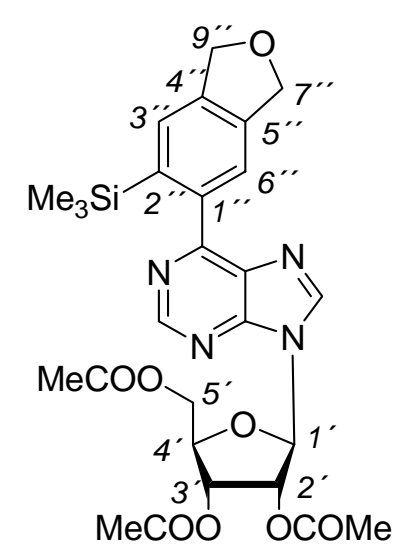

Column chromatography on silica gel (diethyl ether) afforded $17 \mathrm{mg}$ (15\%) of a white foam: $[\alpha]_{\mathrm{D}}+25.8\left(\right.$ (c $\left.0.21, \mathrm{CHCl}_{3}\right)$; ${ }^{1} \mathrm{H}$ NMR (400 $\left.\mathrm{MHz}, \mathrm{CDCl}_{3}\right) \delta 0.18\left(\mathrm{~s}, 9 \mathrm{H},\left(\mathrm{CH}_{3}\right)_{3} \mathrm{Si}\right), 2.11,2.13$ and $2.17(3 \times \mathrm{s}$, $\left.3 \times 3 \mathrm{H}, \mathrm{CH}_{3} \mathrm{CO}\right), 4.41\left(\mathrm{dd}, 1 \mathrm{H}, J_{\mathrm{gem}}=12.9, J_{5^{\prime} \mathrm{b}, 4^{\prime}}=5.2, \mathrm{H}-5^{\prime} \mathrm{b}\right), 4.47-$ 4.52 (m, 2H, H-5'a and H-4'), 5.21 (m, 4H, H-7" and H-9"), 5.73 (dd, $\left.1 \mathrm{H}, J_{3^{\prime}, 2^{\prime}}=5.6, J_{3^{\prime}, 4^{\prime}}=4.7, \mathrm{H}-3^{\prime}\right), 6.01\left(\mathrm{t}, 1 \mathrm{H}, J_{2^{\prime}, 3^{\prime}}=5.6, J_{2^{\prime}, 1^{\prime}}=5.1\right.$, $\left.\mathrm{H}-2^{\prime}\right), 6.31\left(\mathrm{~d}, 1 \mathrm{H}, J_{1^{\prime}, 2^{\prime}}=5.1, \mathrm{H}-1^{\prime}\right), 7.70$ (s, 1H, H-3"), 8.27 (s, 2H, $\mathrm{H}-8$ and $\mathrm{H}-6 "), 8.98$ (s, $1 \mathrm{H}, \mathrm{H}-2)$; $\left.{ }^{13} \mathrm{C} \mathrm{NMR} \mathrm{(100.6} \mathrm{MHz,} \mathrm{CDCl}_{3}\right) \delta$ $1.59\left(\left(\mathrm{CH}_{3}\right)_{3} \mathrm{Si}\right), 20.40,20.53$ and $20.76\left(\mathrm{CH}_{3} \mathrm{CO}\right), 63.00\left(\mathrm{CH}_{2}-5^{\prime}\right), 70.54\left(\mathrm{CH}-3^{\prime}\right), 73.21$ (CH-2'), $73.57\left(\mathrm{CH}_{2}-7^{\prime \prime}\right.$ and $\left.\mathrm{CH}_{2}-9^{\prime \prime}\right), 80.35\left(\mathrm{CH}-4^{\prime}\right), 86.55\left(\mathrm{CH}-1^{\prime}\right), 124.11\left(\mathrm{CH}-6^{\prime \prime}\right), 128.60$ (CH-3"), 131.89 (C-5), 140.09, 140.35, 140.67 and 140.78 (C-1", C-2", C-4" and C-5"), 142.62 (CH-8), 151.60 (CH-2), 151.62 (C-4), 158.65 (C-6), 169.36, 169.56 and 170.28 (CO); IR $\left(\mathrm{CHCl}_{3}\right) \vee 3008,1751,1586,1374,1247,1048 \mathrm{~cm}^{-1}$. MS (FAB, m/z (rel.\%)): $569\left(\mathrm{M}^{+}+\mathrm{H}\right.$, 15), 285 (8), 266 (7), 73 (100). HR-MS (FAB) calcd for $\mathrm{C}_{27} \mathrm{H}_{33} \mathrm{~N}_{4} \mathrm{O}_{8} \mathrm{Si}\left[\mathrm{M}^{+}+\mathrm{H}\right]$ 569.2068, found 569.2087. R $($ hexane/EtOAc 1/2) $=0.37$. (Method C, yield 55\%.) 
IV. Deprotection of the Acetylated 9-( $\beta$-D-Ribofuranosyl)-6-arylpurines.

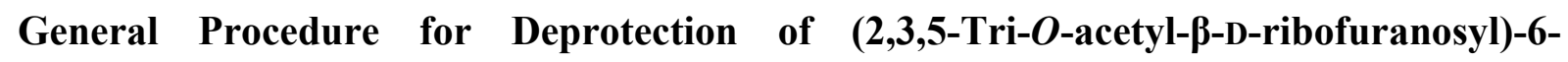
arylpurines.

The prepared 6-aryl-9-(2,3,5-tri-O-acetyl- $\beta$-D-ribofuranosyl)-9H-purine $\mathbf{3 x y}(0.2 \mathrm{mmol})$ in methanol $(8 \mathrm{ml})$ was treated with $1 \mathrm{M}$ methanolic sodium methoxide $(40 \mu 1,0.040 \mathrm{mmol})$ at room temperature for $1 \mathrm{~h}$. The mixture was evaporated with silica gel and chromatographed on a silica column affording the corresponding 6-aryl-9-( $\beta$-D-ribofuranosyl)-9H-purine $\mathbf{4 x y}$.

\section{6-[6-Phenyl-2,2-di(carboxyethyl)indan-5-yl]-9-( $\beta$-D-ribofuranosyl)-9H-purine (4aa).}

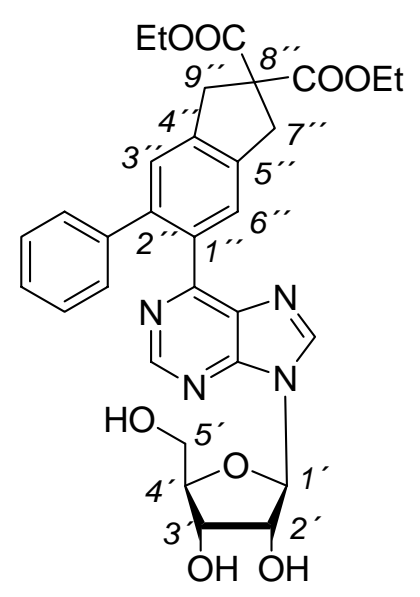

Column chromatography on silica gel (EtOAc) afforded $90 \mathrm{mg}$ (76\%) of a white foam: $[\alpha]_{\mathrm{D}}-30.4$ (c $\left.0.13, \mathrm{MeOH}\right) ;{ }^{1} \mathrm{H}$ NMR $(500$ MHz, DMSO- $\left.d_{6}\right) \delta 1.21\left(\mathrm{t}, 6 \mathrm{H}, J_{\text {vic }}=7.1, \mathrm{CH}_{3} \mathrm{CH}_{2}\right), 3.56(\mathrm{ddd}, 1 \mathrm{H}$, $\left.J_{\text {gem }}=12.0, J_{5^{\prime} \mathrm{b}, \mathrm{OH}}=6.0, J_{5^{\prime} \mathrm{b}, 4^{\prime}}=4.1, \mathrm{H}-5^{\prime} \mathrm{b}\right), 3.61\left(\mathrm{~s}, 2 \mathrm{H}, \mathrm{H}-7^{\prime \prime}\right)$, $3.64\left(\mathrm{~s}, 2 \mathrm{H}, \mathrm{H}-9^{\prime \prime}\right), 3.67\left(\mathrm{ddd}, 1 \mathrm{H}, J_{\mathrm{gem}}=12.0, J_{5^{\prime} \mathrm{a}, \mathrm{OH}}=5.1, J_{5^{\prime} \mathrm{a}, 4^{\prime}}=\right.$ 4.1, H-5'a), 3.96 (q, $\left.1 \mathrm{H}, J_{4^{\prime}, 5^{\prime}}=4.1, J_{4^{\prime}, 3^{\prime}}=3.5, \mathrm{H}-4^{\prime}\right), 4.15(\mathrm{td}, 1 \mathrm{H}$, $\left.J_{3^{\prime}, 2^{\prime}}=5.8, J_{3^{\prime}, \mathrm{OH}}=5.0, J_{3^{\prime}, 4^{\prime}}=3.5, \mathrm{H}-3^{\prime}\right), 4.20\left(\mathrm{q}, 4 \mathrm{H}, J_{\text {vic }}=7.1\right.$, $\left.\mathrm{CH}_{2} \mathrm{CH}_{3}\right), 4.58\left(\mathrm{q}, 1 \mathrm{H}, J_{2^{\prime}, \mathrm{OH}}=6.1, J_{2^{\prime}, 1^{\prime}}=5.8, J_{2^{\prime}, 3^{\prime}}=5.8, \mathrm{H}-2^{\prime}\right)$, $5.07\left(\mathrm{t}, 1 \mathrm{H}, J_{\mathrm{OH}, 5^{\prime}}=6.0,5.1, \mathrm{OH}-5^{\prime}\right), 5.22\left(\mathrm{~d}, 1 \mathrm{H}, J_{\mathrm{OH}, 3^{\prime}}=5.0, \mathrm{OH}-\right.$ $\left.3^{\prime}\right), 5.49\left(\mathrm{~d}, 1 \mathrm{H}, J_{\mathrm{OH}, 2^{\prime}}=6.1, \mathrm{OH}-2^{\prime}\right), 6.00\left(\mathrm{~d}, 1 \mathrm{H}, J_{1^{\prime}, 2^{\prime}}=5.8, \mathrm{H}-1^{\prime}\right), 7.03(\mathrm{~m}, 2 \mathrm{H}, \mathrm{H}-\mathrm{o}-\mathrm{Ph})$, 7.09-7.17 (m, 3H, H-m,p-Ph), 7.40 (s, 1H, H-3"), 7.54 (s, 1H, H-6"), 8.68 (s, 1H, H-8), 8.75 (s, 1H, H-2); ${ }^{13} \mathrm{C}$ NMR (125.8 MHz, DMSO-d $) \delta 14.02\left(\mathrm{CH}_{3} \mathrm{CH}_{2}\right), 39.51\left(\mathrm{CH}_{2}-7^{\prime \prime}\right.$ overlapped by DMSO- $\left.d_{6}\right), 39.94\left(\mathrm{CH}_{2}-7^{\prime \prime}\right.$ overlapped by DMSO- $\left.d_{6}\right), 59.94\left(\mathrm{C}-8^{\prime \prime}\right), 61.44$ $\left(\mathrm{CH}_{2}-5^{\prime}\right), 61.71\left(\mathrm{CH}_{2} \mathrm{CH}_{3}\right), 70.44\left(\mathrm{CH}-3^{\prime}\right), 73.75\left(\mathrm{CH}-2^{\prime}\right), 85.86\left(\mathrm{CH}-4^{\prime}\right), 87.61\left(\mathrm{CH}-1^{\prime}\right)$, 126.38 (CH-3"), 126.67 (CH-p-Ph), 127.03 (CH-6"), 128.00 (CH-m-Ph), 128.93 (CH-o-Ph), 132.46 (C-5), 133.42 (C-1"), 138.91 (C-5"), 140.57 (C-2"), 141.01 (C-i-Ph), 141.94 (C-4"), 144.79 (CH-8), 151.32 (C-4), 151.65 (CH-2), 158.33 (C-6), 171.02 (CO);KBr) v 3296, 3235, 1731, 1589, 1248, $1072 \mathrm{~cm}^{-1}$; MS (FAB, m/z (rel.\%)) $589\left(\mathrm{M}^{+}+\mathrm{H}, 26\right), 457$ (100); HR-MS (FAB) calcd for $\mathrm{C}_{31} \mathrm{H}_{33} \mathrm{~N}_{4} \mathrm{O}_{8}\left[\mathrm{M}^{+}+\mathrm{H}\right]$ 589.2298, found 589.2296. $\mathrm{R}_{f}($ EtOAc/MeOH 10/1) $=$ 0.47 .

6-[6-Phenyl-2-acetyl-2-(carboxyethyl)indan-5-yl]-9-( $\beta$-D-ribofuranosyl)-9H-purine (4ac). Column chromatography on silica gel (EtOAc/MeOH 10/1) afforded $92 \mathrm{mg}(82 \%)$ of a white foam: $[\alpha]_{\mathrm{D}}-40.1$ (c 0.19, MeOH); ${ }^{1} \mathrm{H}$ NMR (500 MHz, DMSO- $\left.d_{6}\right) \delta 1.22\left(\mathrm{t}, 3 \mathrm{H}, J_{\mathrm{vic}}=7.1\right.$, 


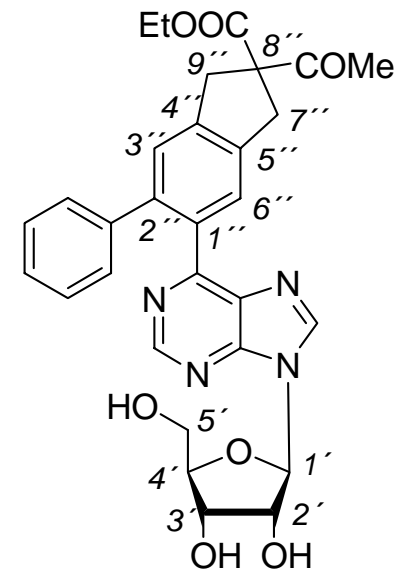

$\mathrm{CH}_{3} \mathrm{CH}_{2}$ ), 2.26 (s, 3H, $\left.\mathrm{CH}_{3} \mathrm{CO}\right), 3.51-3.70\left(\mathrm{~m}, 6 \mathrm{H}, \mathrm{H}-5^{\prime}, \mathrm{H}-7^{\prime \prime}\right.$ and $\left.\mathrm{H}-9^{\prime \prime}\right), 3.96\left(\mathrm{q}, 1 \mathrm{H}, J_{4^{\prime}, 5^{\prime}}=4.0, J_{4^{\prime}, 3^{\prime}}=3.6, \mathrm{H}-4^{\prime}\right), 4.15\left(\mathrm{td}, 1 \mathrm{H}, J_{3^{\prime}, \mathrm{OH}}\right.$ $\left.=5.0, J_{3^{\prime}, 2^{\prime}}=4.9, J_{3^{\prime}, 4^{\prime}}=3.6, \mathrm{H}-3^{\prime}\right), 4.21\left(\mathrm{q}, 2 \mathrm{H}, J_{\text {vic }}=7.1, \mathrm{CH}_{2} \mathrm{CH}_{3}\right)$, $4.59\left(\mathrm{q}, 1 \mathrm{H}, J_{2^{\prime}, \mathrm{OH}}=6.2, J_{2^{\prime}, 1^{\prime}}=5.8, J_{2^{\prime}, 3^{\prime}}=4.9, \mathrm{H}-2^{\prime}\right), 5.09(\mathrm{t}, 1 \mathrm{H}$, $\left.J_{\mathrm{OH}, 5^{\prime}}=5.5, \mathrm{OH}-5^{\prime}\right), 5.25\left(\mathrm{~d}, 1 \mathrm{H}, J_{\mathrm{OH}, 3^{\prime}}=5.0, \mathrm{OH}-3^{\prime}\right), 5.51(\mathrm{~d}, 1 \mathrm{H}$, $\left.J_{\mathrm{OH}, 2^{\prime}}=6.2, \mathrm{OH}-2^{\prime}\right), 6.00\left(\mathrm{~d}, 1 \mathrm{H}, J_{1^{\prime}, 2^{\prime}}=5.8, \mathrm{H}-1^{\prime}\right), 7.02(\mathrm{~m}, 2 \mathrm{H}, \mathrm{H}-$ o-Ph), 7.09-7.17 (m, 3H, H-m,p-Ph), 7.38 (s, 1H, H-3"), 7.53 (s, 1H,

H-6"), 8.68 (s, 1H, H-8), 8.75 (s, 1H, H-2); ${ }^{13} \mathrm{C} \mathrm{NMR} \mathrm{(125.8} \mathrm{MHz,}$ DMSO-d $\left.d_{6}\right) \delta 14.04\left(\mathrm{CH}_{3} \mathrm{CH}_{2}\right), 26.33\left(\mathrm{CH}_{3} \mathrm{CO}\right), 38.20$ and $38.49\left(\mathrm{CH}_{2}-7^{\prime \prime}\right.$ and $\left.\mathrm{CH}_{2}-9^{\prime \prime}\right), 61.46$ $\left(\mathrm{CH}_{2}-5^{\prime}\right), 61.84\left(\mathrm{CH}_{2} \mathrm{CH}_{3}\right), 66.54\left(\mathrm{C}-8^{\prime \prime}\right), 70.47\left(\mathrm{CH}-3^{\prime}\right), 73.77\left(\mathrm{CH}-2^{\prime}\right), 85.89\left(\mathrm{CH}-4^{\prime}\right), 87.61$ (CH-1'), 126.46 (CH-3"), 126.69 (CH-p-Ph), 127.12 (CH-6"), 128.04 (CH-m-Ph), 128.96 (CH-o-Ph), 132.48 (C-5), 133.36 (C-1"), 139.11 (C-5"), 140.52 (C-2"), 141.08 (C-i-Ph), 142.15 (C-4"), 144.85 (CH-8), 151.35 (C-4), 151.69 (CH-2), 158.38 (C-6), 171.90 (COOEt), 202.77 (COMe); IR (KBr) v 3418, 1734, 1713, 1587, 1329, 1237, 1216, 1088, $1074 \mathrm{~cm}^{-1}$; MS (FAB, m/z (rel.\%)) $559\left(\mathrm{M}^{+}+\mathrm{H}, 55\right), 427$ (100); HR-MS (FAB) calcd for $\mathrm{C}_{31} \mathrm{H}_{33} \mathrm{~N}_{4} \mathrm{O}_{8}$ $\left[\mathrm{M}^{+}+\mathrm{H}\right]$ 559.2193, found 559.2214. $\mathrm{R}_{f}(\mathrm{EtOAc} / \mathrm{MeOH} 10 / 1)=0.47$.

\section{6-[6-Phenyl-2-cyano-2-(carboxymethyl)indan-5-yl]-9-( $\beta$-D-ribofuranosyl)-9H-purine}

(4ad).

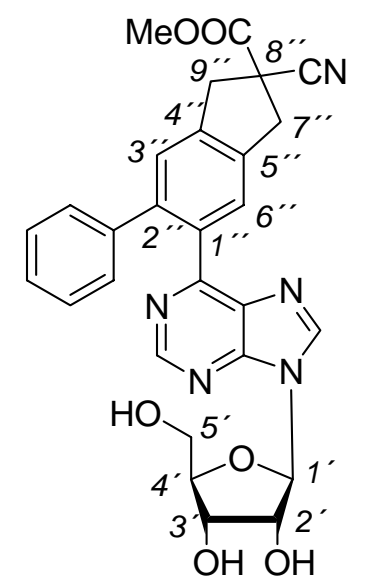

Column chromatography on silica gel (EtOAc) afforded $65 \mathrm{mg}(60 \%)$ of a white amorphous solid: $\mathrm{mp} 121-126^{\circ} \mathrm{C} ;[\alpha]_{\mathrm{D}}-42.3($ c 0.15 , $\mathrm{MeOH}) ;{ }^{1} \mathrm{H}$ NMR $\left(500 \mathrm{MHz}, \mathrm{DMSO}-d_{6}\right) \delta 3.56\left(\mathrm{ddd}, 1 \mathrm{H}, J_{\mathrm{gem}}=12.0\right.$, $\left.J_{5^{\prime} \mathrm{b}, \mathrm{OH}}=6.0, J_{5^{\prime} \mathrm{b}, 4^{\prime}}=4.1, \mathrm{H}-5^{\prime} \mathrm{b}\right), 3.67\left(\mathrm{ddd}, 1 \mathrm{H}, J_{\mathrm{gem}}=12.0, J_{5^{\prime} \mathrm{a}, \mathrm{OH}}=\right.$ $\left.5.0, J_{5^{\prime} \mathrm{a}, 4^{\prime}}=4.1, \mathrm{H}-5^{\prime} \mathrm{a}\right), 3.71,3.74,3.76$ and $3.79\left(4 \times \mathrm{d}, 4 \mathrm{H}, J_{\mathrm{gem}}=16.5\right.$, H-7" and H-9"), $3.84\left(\mathrm{~s}, 3 \mathrm{H}, \mathrm{CH}_{3} \mathrm{O}\right), 3.96\left(\mathrm{q}, 1 \mathrm{H}, J_{4^{\prime}, 5^{\prime}}=4.1, J_{4^{\prime}, 3^{\prime}}=3.7\right.$, $\left.\mathrm{H}-4^{\prime}\right), 4.15\left(\mathrm{td}, 1 \mathrm{H}, J_{3^{\prime}, 2^{\prime}}=4.9, J_{3^{\prime}, \mathrm{OH}}=4.9, J_{3^{\prime}, 4^{\prime}}=3.7, \mathrm{H}-3^{\prime}\right), 4.59$ (q, $\left.1 \mathrm{H}, J_{2^{\prime}, \mathrm{OH}}=6.1, J_{2^{\prime}, 1^{\prime}}=5.8, J_{2^{\prime}, 3^{\prime}}=4.9, \mathrm{H}-2^{\prime}\right), 5.07\left(\mathrm{t}, 1 \mathrm{H}, J_{\mathrm{OH}, 5^{\prime}}=6.0\right.$, 5.0, OH-5') $5.22\left(\mathrm{~d}, 1 \mathrm{H}, J_{\mathrm{OH}, 3^{\prime}}=4.9, \mathrm{OH}-3^{\prime}\right), 5.49\left(\mathrm{~d}, 1 \mathrm{H}, J_{\mathrm{OH}, 2^{\prime}}=6.1, \mathrm{OH}-2^{\prime}\right), 6.00(\mathrm{~d}, 1 \mathrm{H}$, $\left.J_{1^{\prime}, 2^{\prime}}=5.8, \mathrm{H}-1^{\prime}\right), 7.05$ (m, 2H, H-o-Ph), 7.11-7.18 (m, 3H, H-m,p-Ph), 7.47 (s, 1H, H-3"), 7.62 (s, 1H, H-6"), 8.69 (s, 1H, H-8), 8.77 (s, 1H, H-2); ${ }^{13} \mathrm{C}$ NMR (125.8 MHz, DMSO-d 6 ) $\delta$ 42.41 and $42.62\left(\mathrm{CH}_{2}-7^{\prime \prime}\right.$ and $\left.\mathrm{CH}_{2}-9^{\prime \prime}\right), 47.62\left(\mathrm{C}-8^{\prime \prime}\right), 54.11\left(\mathrm{CH}_{3} \mathrm{O}\right), 61.46\left(\mathrm{CH}_{2}-5^{\prime}\right), 70.44$ (CH-3'), 73.77 (CH-2'), $85.86\left(\mathrm{CH}^{\prime} 4^{\prime}\right), 87.63\left(\mathrm{CH}^{\prime} 1^{\prime}\right), 120.95(\mathrm{CN}), 126.65\left(\mathrm{CH}-3^{\prime \prime}\right), 126.82$ (CH-p-Ph), 127.28 (CH-6"), 128.05 (CH-m-Ph), 128.93 (CH-o-Ph), 132.45 (C-5), 133.90 (C- 
1"), 137.67 (C-5"), 140.67 and 140.78 (C-2" and C-4"), 141.09 (C-i-Ph), 144.90 (CH-8), 151.38 (C-4), 151.68 (CH-2), 158.02 (C-6), 168.68 (CO); IR (KBr) v 3295, 2246, 1747, 1588, 1435, 1330, 1240, 1215, 1088, 1074, $1057 \mathrm{~cm}^{-1}$; MS (FAB, m/z (rel.\%)) $528\left(\mathrm{M}^{+}+\mathrm{H}\right.$, 18), 396 (100); HR-MS (FAB) calcd for $\mathrm{C}_{28} \mathrm{H}_{26} \mathrm{~N}_{5} \mathrm{O}_{6}\left[\mathrm{M}^{+}+\mathrm{H}\right]$ 528.1883, found 528.1897. $\mathrm{R}_{f}(\mathrm{EtOAc} / \mathrm{MeOH} 10 / 1)=0.40$.

\section{6-[6-Phenyl-1,3-dihydro-2-tosyl-2H-indol-5-yl)]-9-( $\beta$-D-ribofuranosyl)-9H-purine (4ae).}

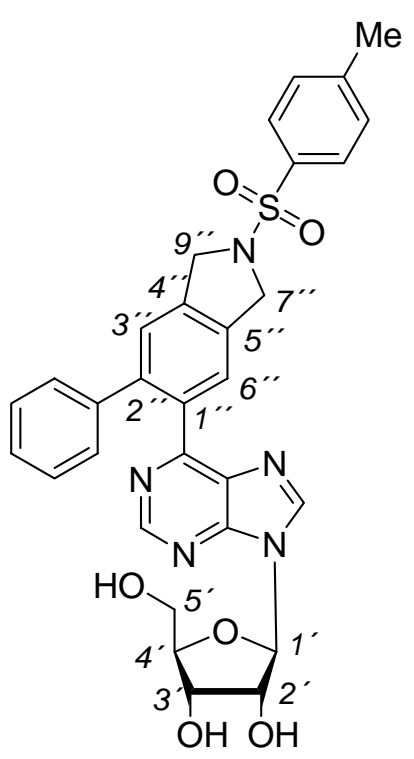

Column chromatography on silica gel (EtOAc) afforded $78 \mathrm{mg}$ $(65 \%)$ of a white amorphous solid: $\mathrm{mp} 147-152^{\circ} \mathrm{C} ;[\alpha]_{\mathrm{D}}-33.5(\mathrm{c}$ $0.18, \mathrm{MeOH}) ;{ }^{1} \mathrm{H} \mathrm{NMR}\left(400 \mathrm{MHz}, \mathrm{DMSO}-d_{6}\right) \delta 2.38\left(\mathrm{~s}, 3 \mathrm{H}, \mathrm{CH}_{3}\right)$, $3.54\left(\mathrm{ddd}, 1 \mathrm{H}, J_{\mathrm{gem}}=12.2, J_{5^{\prime} \mathrm{b}, \mathrm{OH}}=6.1, J_{5^{\prime} \mathrm{b}, 4^{\prime}}=4.3, \mathrm{H}-5^{\prime} \mathrm{b}\right), 3.66$ $\left(\mathrm{ddd}, 1 \mathrm{H}, J_{\mathrm{gem}}=12.2, J_{5^{\prime} \mathrm{a}, \mathrm{OH}}=5.2, J_{5^{\prime} \mathrm{a}, 4^{\prime}}=4.2, \mathrm{H}-5^{\prime} \mathrm{a}\right), 3.95(\mathrm{q}, 1 \mathrm{H}$, $\left.J_{4^{\prime}, 5^{\prime} \mathrm{b}}=4.3, J_{4^{\prime}, 5^{\prime} \mathrm{a}}=4.2, J_{4^{\prime}, 3^{\prime}}=3.8, \mathrm{H}-4^{\prime}\right), 4.14\left(\mathrm{q}, 1 \mathrm{H}, J_{3^{\prime}, 2^{\prime}}=5.4\right.$, $\left.J_{3^{\prime}, \mathrm{OH}}=4.7, J_{3^{\prime}, 4^{\prime}}=3.8, \mathrm{H}-3^{\prime}\right), 4.57\left(\mathrm{q}, 1 \mathrm{H}, J_{2^{\prime}, \mathrm{OH}}=6.1, J_{2^{\prime}, 1^{\prime}}=5.8\right.$, $\left.J_{2^{\prime}, 3^{\prime}}=5.4, \mathrm{H}-2^{\prime}\right), 4.68$ and $4.70\left(2 \times \mathrm{bs}, 2 \times 2 \mathrm{H}, \mathrm{H}-7^{\prime \prime}\right.$ and $\left.\mathrm{H}-9^{\prime \prime}\right), 5.08$ $\left(\mathrm{t}, 1 \mathrm{H}, J_{\mathrm{OH}, 5^{\prime} \mathrm{b}}=6.1, J_{\mathrm{OH}, 5^{\prime} \mathrm{a}}=5.2, \mathrm{OH}-5^{\prime}\right), 5.25\left(\mathrm{~d}, 1 \mathrm{H}, J_{\mathrm{OH}, 3^{\prime}}=4.7\right.$, $\left.\mathrm{OH}-3^{\prime}\right), 5.51\left(\mathrm{~d}, 1 \mathrm{H}, J_{\mathrm{OH}, 2^{\prime}}=6.1, \mathrm{OH}-2^{\prime}\right), 5.98\left(\mathrm{~d}, 1 \mathrm{H}, J_{1^{\prime}, 2^{\prime}}=5.8\right.$, H-1'), 6.99 (m, 2H, m-H-Ph), 7.10-7.15 (m, 3H, o-+ p-H-Ph), 7.41 (s, 1H, H-6"), 7.45 (m, 2H, m-H-Tos), 7.55 (s, 1H, H-3"), 7.80 (m, 2H, o-H-Tos), 8.67 (s, 1H, $\mathrm{H}-8), 8.75$ (s, 1H, H-2); ${ }^{13} \mathrm{C}$ NMR (100.6 MHz, DMSO-d $\left.d_{6}\right) \delta 21.20\left(\mathrm{CH}_{3}\right), 53.50$ and 53.67 $\left(\mathrm{CH}_{2}-7^{\prime \prime}\right.$ and $\left.\mathrm{CH}_{2}-9^{\prime \prime}\right), 61.42\left(\mathrm{CH}_{2}-5^{\prime}\right), 70.43\left(\mathrm{CH}-3^{\prime}\right), 73.76\left(\mathrm{CH}-2^{\prime}\right), 85.87\left(\mathrm{CH}-4^{\prime}\right), 87.61$ (CH-1'), 125.08 (CH-6"), 125.65 (CH-3"), 126.94 (CH-p-Ph), 127.68 (CH-o-Tos), 128.08 (CH-o-Ph), 128.90 (CH-m-Ph), 130.27 (CH-m-Tos), 132.43 (C-5), 133.06 (C-i-Tos), 134.12 and 135.06 (C-2" and C-4"), 137.90 (C-5"), 140.57 (C-i-Ph), 141.23 (C-1"), 144.04 (C-p-Tos), 144.99 (CH-8), 151.36 (C-4), 151.70 (CH-2), 157.77 (C-6); IR (KBr) v 3429, 3260, 1587, 1332, 1162, 1096, 1074, $1056 \mathrm{~cm}^{-1}$; MS (FAB, m/z (rel.\%)) $600\left(\mathrm{M}^{+}+\mathrm{H}, 13\right), 468$ (100); HR-MS (FAB) calcd for $\mathrm{C}_{31} \mathrm{H}_{30} \mathrm{~N}_{5} \mathrm{O}_{6} \mathrm{~S}\left[\mathrm{M}^{+}+\mathrm{H}\right] 600.1917$, found 600.1938. $\mathrm{R}_{f}(\mathrm{EtOAc} / \mathrm{MeOH}$ $10 / 1)=0.36$.

\section{6-[6-Phenyl-1,3-dihydroisobenzofuran-5-yl]-9-( $\beta$-D-ribofuranosyl)-9H-purine (4ag).}

Column chromatography on silica gel (EtOAc/MeOH 10/1) afforded $76 \mathrm{mg}$ (85\%) of a white amorphous solid: mp $138-141^{\circ} \mathrm{C}$; $[\alpha]_{\mathrm{D}}-36.6$ (c 0.14, MeOH); ${ }^{1} \mathrm{H}$ NMR (400 MHz, DMSO$\left.d_{6}\right) \delta 3.56\left(\mathrm{ddd}, 1 \mathrm{H}, J_{\mathrm{gem}}=12.0, J_{5^{\prime} \mathrm{b}, \mathrm{OH}}=6.0, J_{5^{\prime} \mathrm{b}, 4^{\prime}}=4.1, \mathrm{H}-5^{\prime} \mathrm{b}\right), 3.67\left(\mathrm{ddd}, 1 \mathrm{H}, J_{\mathrm{gem}}=12.0\right.$, 


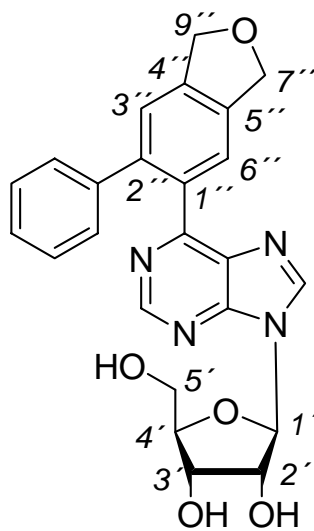

$\left.J_{5^{\prime} \mathrm{a}, \mathrm{OH}}=5.2, J_{5^{\prime} \mathrm{a}, 4^{\prime}}=4.1, \mathrm{H}-5^{\prime} \mathrm{a}\right), 3.96\left(\mathrm{q}, 1 \mathrm{H}, J_{4^{\prime}, 5^{\prime}}=4.1, J_{4^{\prime}, 3^{\prime}}=3.4, \mathrm{H}-\right.$ $\left.4^{\prime}\right), 4.15\left(\mathrm{td}, 1 \mathrm{H}, J_{3^{\prime}, 2^{\prime}}=5.1, J_{3^{\prime}, \mathrm{OH}}=5.0, J_{3^{\prime}, 4^{\prime}}=3.4, \mathrm{H}-3^{\prime}\right), 4.59(\mathrm{q}, 1 \mathrm{H}$, $\left.J_{2^{\prime}, \mathrm{OH}}=6.2, J_{2^{\prime}, 1^{\prime}}=5.8, J_{2^{\prime}, 3^{\prime}}=5.1, \mathrm{H}-2^{\prime}\right), 5.09\left(\mathrm{t}, 1 \mathrm{H}, J_{\mathrm{OH}, 5^{\prime}}=6.0,5.2\right.$, $\left.\mathrm{OH}-5^{\prime}\right), 5.13\left(\mathrm{~m}, 4 \mathrm{H}, \mathrm{H}-7^{\prime \prime}\right.$ and $\left.\mathrm{H}-9^{\prime \prime}\right), 5.25$ (d, 1H, $\left.J_{\mathrm{OH}, 3^{\prime}}=5.0, \mathrm{OH}-3^{\prime}\right)$, $5.51\left(\mathrm{~d}, 1 \mathrm{H}, J_{\mathrm{OH}, 2^{\prime}}=6.2, \mathrm{OH}-2^{\prime}\right), 6.00\left(\mathrm{~d}, 1 \mathrm{H}, J_{1^{\prime}, 2^{\prime}}=5.8, \mathrm{H}-1^{\prime}\right), 7.06(\mathrm{~m}$, 2H, H-o-Ph), 7.10-7.19 (m, 3H, H-m,p-Ph), 7.48 (s, 1H, H-3"), 7.63 (s, $1 \mathrm{H}, \mathrm{H}-6 "), 8.70$ (s, 1H, H-8), 8.77 (s, 1H, H-2); ${ }^{13} \mathrm{C}$ NMR (100.6 MHz, DMSO-d $\left.{ }_{6}\right) \delta 61.45\left(\mathrm{CH}_{2}-5^{\prime}\right), 70.46\left(\mathrm{CH}-3^{\prime}\right), 72.57$ and $72.72\left(\mathrm{H}-7^{\prime \prime}\right.$ and H-9"), 73.76 (CH-2'), 85.88 (CH-4'), 87.62 (CH-1'), 123.39 (CH-3"), 124.06 (CH-6"), 126.81 (CH-p-Ph), 128.06 (CH-m-Ph), 129.01 (CH-o-Ph), 132.54 (C-5), 133.77 (C-1"), 138.24 (C5"), 140.97 and 140.98 (C-2" and C-i-Ph), 141.18 (C-4"), 144.93 (CH-8), 151.37 (C-4), 151.69 $(\mathrm{CH}-2), 158.24$ (C-6); IR (KBr) $\vee 3435,1589,1331,1208,1088,1076,1048 \mathrm{~cm}^{-1}$; MS $\left(\mathrm{FAB}, \mathrm{m} / \mathrm{z}\left(\right.\right.$ rel.\%)) $447\left(\mathrm{M}^{+}+\mathrm{H}, 69\right), 315$ (100); HR-MS (FAB) calcd for $\mathrm{C}_{24} \mathrm{H}_{23} \mathrm{~N}_{4} \mathrm{O}_{5}$ $\left[\mathrm{M}^{+}+\mathrm{H}\right]$ 447.1668, found 447.1658. $\mathrm{R}_{f}($ EtOAc/ $\mathrm{MeOH} 5 / 1)=0.59$.

\section{6-[6-Butyl-2,2-di(carboxyethyl)indan-5-yl]-9-(ß-D-ribofuranosyl)-9H-purine (4ba).}

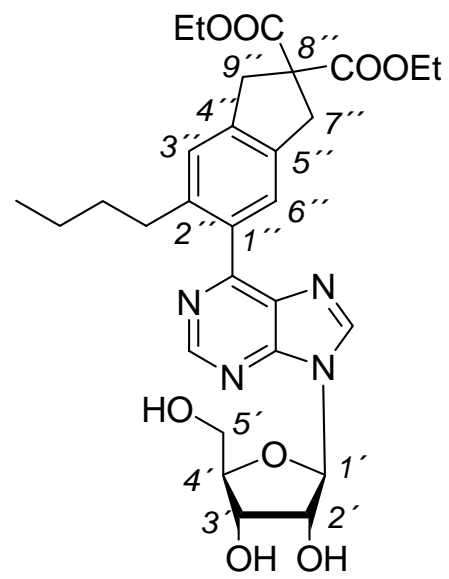

Column chromatography on silica gel (EtOAc) afforded $100 \mathrm{mg}$ (88\%) of a white amorphous solid: $\mathrm{mp} 65-68^{\circ} \mathrm{C} ; \quad[\alpha]_{\mathrm{D}}-24.2(\mathrm{c}$ $0.14, \mathrm{MeOH}) ;{ }^{1} \mathrm{H} \mathrm{NMR}\left(500 \mathrm{MHz}, \mathrm{DMSO}-d_{6}\right) \delta 0.65\left(\mathrm{t}, 3 \mathrm{H}, J_{\text {vic }}\right.$ $\left.=7.3, \mathrm{CH}_{3} \mathrm{CH}_{2} \mathrm{CH}_{2} \mathrm{CH}_{2}\right), 1.09\left(\mathrm{~m}, 2 \mathrm{H}, \mathrm{CH}_{3} \mathrm{CH}_{2} \mathrm{CH}_{2} \mathrm{CH}_{2}\right), 1.19$ (t, $\left.6 \mathrm{H}, J_{\text {vic }}=7.1, \mathrm{CH}_{3} \mathrm{CH}_{2}\right), 1.32\left(\mathrm{~m}, 2 \mathrm{H}, \mathrm{CH}_{3} \mathrm{CH}_{2} \mathrm{CH}_{2} \mathrm{CH}_{2}\right), 2.68$ (m, 2H, $\mathrm{CH}_{3} \mathrm{CH}_{2} \mathrm{CH}_{2} \mathrm{CH}_{2}$ ), 3.52 (bs, 2H, H-7"), 3.56 (bs, 2H, H$\left.9^{\prime \prime}\right), 3.59\left(\mathrm{ddd}, 1 \mathrm{H}, J_{\mathrm{gem}}=12.1, J_{5^{\prime} \mathrm{b}, \mathrm{OH}}=6.0, J_{5^{\prime} \mathrm{b}, 4^{\prime}}=4.1, \mathrm{H}-5^{\prime} \mathrm{b}\right)$, $3.70\left(\mathrm{ddd}, 1 \mathrm{H}, J_{\mathrm{gem}}=12.1, J_{5^{\prime} \mathrm{a}, \mathrm{OH}}=5.2, J_{5^{\prime} \mathrm{a}, 4^{\prime}}=4.2, \mathrm{H}-5^{\prime} \mathrm{a}\right), 4.00$ $\left(\mathrm{q}, 1 \mathrm{H}, J_{4^{\prime}, 5^{\prime}}=4.2,4.1, J_{4^{\prime}, 3^{\prime}}=3.4, \mathrm{H}-4^{\prime}\right), 4.17\left(\mathrm{q}, 4 \mathrm{H}, J_{\text {vic }}=7.1, \mathrm{CH}_{2} \mathrm{CH}_{3}\right), 4.20\left(\mathrm{td}, 1 \mathrm{H}, J_{3^{\prime}, \mathrm{OH}}\right.$ $\left.=5.0, J_{3^{\prime}, 2^{\prime}}=4.8, J_{3^{\prime}, 4^{\prime}}=3.4, \mathrm{H}-3^{\prime}\right), 4.67\left(\mathrm{q}, 1 \mathrm{H}, J_{2^{\prime}, \mathrm{OH}}=6.0, J_{2^{\prime}, 1^{\prime}}=5.8, J_{2^{\prime}, 3^{\prime}}=4.8, \mathrm{H}-2^{\prime}\right)$, $5.12\left(\mathrm{t}, 1 \mathrm{H}, J_{\mathrm{OH}, 5^{\prime}}=6.0,5.2, \mathrm{OH}-5^{\prime}\right), 5.27\left(\mathrm{~d}, 1 \mathrm{H}, J_{\mathrm{OH}, 3^{\prime}}=5.0, \mathrm{OH}-3^{\prime}\right), 5.57\left(\mathrm{~d}, 1 \mathrm{H}, J_{\mathrm{OH}, 2^{\prime}}=\right.$ 6.0, OH-2'), $6.08\left(\mathrm{~d}, 1 \mathrm{H}, J_{1^{\prime}, 2^{\prime}}=5.8, \mathrm{H}^{\prime} 1^{\prime}\right), 7.27$ (s, 1H, H-3"), 7.40 (s, 1H, H-6"), 8.82 (s, 1H, $\mathrm{H}-8), 8.98(\mathrm{~s}, 1 \mathrm{H}, \mathrm{H}-2) ;{ }^{13} \mathrm{C} \mathrm{NMR}\left(125.8 \mathrm{MHz}, \mathrm{DMSO}-d_{6}\right) \delta 13.69\left(\mathrm{CH}_{3} \mathrm{CH}_{2} \mathrm{CH}_{2} \mathrm{CH}_{2}\right), 14.05$ $\left(\mathrm{CH}_{3} \mathrm{CH}_{2}\right), 21.97\left(\mathrm{CH}_{3} \mathrm{CH}_{2} \mathrm{CH}_{2} \mathrm{CH}_{2}\right), 32.37\left(\mathrm{CH}_{3} \mathrm{CH}_{2} \mathrm{CH}_{2} \mathbf{C H}_{2}\right), 33.24\left(\mathrm{CH}_{3} \mathrm{CH}_{2} \mathbf{C H}_{2} \mathrm{CH}_{2}\right)$, $39.70\left(\mathrm{CH}_{2}-7^{\prime \prime}\right), 39.99\left(\mathrm{CH}_{2}-9^{\prime \prime}\right), 59.90\left(\mathrm{C}-8^{\prime \prime}\right), 61.46\left(\mathrm{CH}_{2}-5^{\prime}\right), 61.68\left(\mathrm{CH}_{2} \mathrm{CH}_{3}\right), 70.51(\mathrm{CH}-$ 3'), 73.85 (CH-2'), 85.92 (CH-4'), 87.74 (CH-1'), 125.70 (CH-3"), 126.74 (CH-6"), 132.32 (C-5), 133.70 (C-1"); 137.16 (C-5"), 140.58 (C-2"), 141.59 (C-4"), 145.02 (CH-8), 151.59 (C- 
4), 151.80 (CH-2), 158.34 (C-6), 171.12 (COOEt); IR (KBr) v 3398, 1733, 1586, 1249, 1190, 1072, $1053 \mathrm{~cm}^{-1}$; MS (FAB, m/z (rel.\%)) $569\left(\mathrm{M}^{+}+\mathrm{H}, 50\right), 437$ (100); HR-MS (FAB) calcd for $\mathrm{C}_{29} \mathrm{H}_{37} \mathrm{~N}_{4} \mathrm{O}_{8}\left[\mathrm{M}^{+}+\mathrm{H}\right]$ 569.2611, found 569.2600. $\mathrm{R}_{f}(\mathrm{EtOAc} / \mathrm{MeOH} 10 / 1)=0.50$.

\section{6-[6-Trimethylsilyl-2,2-di(carboxyethyl)indan-5-yl]-9-(ß-D-ribofuranosyl)-9H-purine} (4ca).

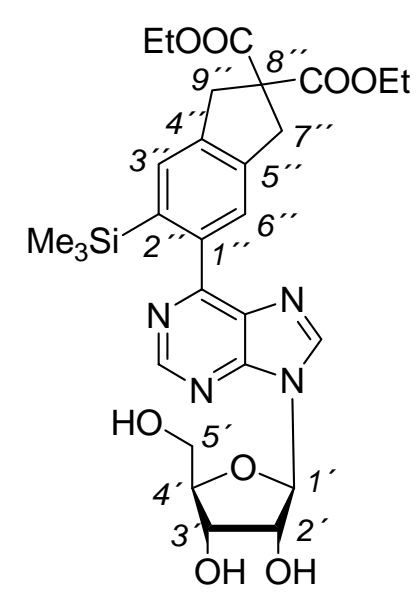

Column chromatography on silica gel (EtOAc) afforded $95 \mathrm{mg}$ (81\%) of a white amorphous solid: $\mathrm{mp} 81-84^{\circ} \mathrm{C} ; \quad[\alpha]_{\mathrm{D}}-9.5($ c 0.19 , $\mathrm{MeOH}) ;{ }^{1} \mathrm{H}$ NMR (400 MHz, DMSO-d $\left.{ }_{6}\right) \delta 0.11\left(\mathrm{~s}, 9 \mathrm{H},\left(\mathrm{CH}_{3}\right)_{3} \mathrm{Si}\right)$, $1.19\left(\mathrm{t}, 6 \mathrm{H}, J_{\text {vic }}=7.1, \mathrm{CH}_{3} \mathrm{CH}_{2}\right), 3.58$ and $3.59\left(2 \times \mathrm{s}, 2 \times 2 \mathrm{H}, \mathrm{H}-7^{\prime \prime}\right.$ and H-9"), $3.60\left(\mathrm{ddd}, 1 \mathrm{H}, J_{\mathrm{gem}}=11.4, J_{5^{\prime} \mathrm{b}, \mathrm{OH}}=5.8, J_{5^{\prime} \mathrm{b}, 4^{\prime}}=4.1, \mathrm{H}-\right.$ $\left.5^{\prime} \mathrm{b}\right), 3.71\left(\mathrm{ddd}, 1 \mathrm{H}, J_{\text {gem }}=11.4, J_{5^{\prime} \mathrm{a}, \mathrm{OH}}=4.9, J_{5^{\prime} \mathrm{a}, 4^{\prime}}=4.1, \mathrm{H}-5^{\prime} \mathrm{a}\right)$, $4.00\left(\mathrm{q}, 1 \mathrm{H}, J_{4^{\prime}, 5^{\prime}}=4.1, J_{4^{\prime}, 3^{\prime}}=3.5, \mathrm{H}-4^{\prime}\right), 4.17\left(\mathrm{q}, 4 \mathrm{H}, J_{\text {vic }}=7.1\right.$, $\left.\mathrm{CH}_{2} \mathrm{CH}_{3}\right), 4.21\left(\mathrm{q}, 1 \mathrm{H}, J_{3^{\prime}, \mathrm{OH}}=5.1, J_{3^{\prime}, 2^{\prime}}=4.7, J_{3^{\prime}, 4^{\prime}}=3.5, \mathrm{H}-3^{\prime}\right)$, $4.66\left(\mathrm{q}, 1 \mathrm{H}, J_{2^{\prime}, \mathrm{OH}}=6.0, J_{2^{\prime}, 1^{\prime}}=5.7, J_{2^{\prime}, 3^{\prime}}=4.7, \mathrm{H}-2^{\prime}\right), 5.13(\mathrm{t}, 1 \mathrm{H}$, $\left.J_{\mathrm{OH}, 5^{\prime}}=5.8,4.9, \mathrm{OH}-5^{\prime}\right), 5.27\left(\mathrm{~d}, 1 \mathrm{H}, J_{\mathrm{OH}, 3^{\prime}}=5.1, \mathrm{OH}-3^{\prime}\right), 5.58\left(\mathrm{~d}, 1 \mathrm{H}, J_{\mathrm{OH}, 2^{\prime}}=6.0, \mathrm{OH}-2^{\prime}\right)$, $6.10\left(\mathrm{~d}, 1 \mathrm{H}, J_{1^{\prime}, 2^{\prime}}=5.7, \mathrm{H}-1^{\prime}\right), 7.65$ (s, 1H, H-3"), 8.25 (s, 1H, H-6"), 8.89 (s, 1H, H-8), 8.97 (s, 1H, H-2); ${ }^{13} \mathrm{C}$ NMR (100.6 MHz, DMSO-d 6$) \delta 1.98\left(\left(\mathrm{CH}_{3}\right)_{3} \mathrm{Si}\right), 14.04\left(\mathrm{CH}_{3} \mathrm{CH}_{2}\right), 40.12$ $\left(\mathrm{CH}_{2}-7^{\prime \prime}\right.$ and $\left.\mathrm{CH}_{2}-9^{\prime \prime}\right), 59.75\left(\mathrm{C}-8^{\prime \prime}\right), 61.41\left(\mathrm{CH}_{2}-5^{\prime}\right), 61.71\left(\mathrm{CH}_{2} \mathrm{CH}_{3}\right), 70.47\left(\mathrm{CH}-3^{\prime}\right), 73.92$ (CH-2'), 85.90 (CH-4'), 87.75 (CH-1'), 127.36 (CH-6"), 131.28 (C-5), 131.73 (CH-3"), 139.27 (C-2"), 140.28 (C-1"), 140.80 (C-5"), 141.52 (C-4"), 145.02 (CH-8), 151.02 (CH-2), 152.02 (C-4), 156.98 (C-6), 171.08 (COOEt); IR (KBr) v 3425, 3270, 1733, 1582, 1247, 1210, 1190, 1071, $1053 \mathrm{~cm}^{-1}$; MS (FAB, m/z (rel.\%)) $585\left(\mathrm{M}^{+}+\mathrm{H}, 15\right), 453$ (32), 73 (100); HR-MS (FAB) calcd for $\mathrm{C}_{28} \mathrm{H}_{37} \mathrm{~N}_{4} \mathrm{O}_{8} \mathrm{Si}\left[\mathrm{M}^{+}+\mathrm{H}\right]$ 585.2381, found 585.2366. $\mathrm{R}_{f}($ EtOAc) $=$ 0.42 . 


\section{Cyclotrimerization of Unprotected 9-( $\beta$-D-Ribofuranosyl)-6-alkynylpurines.}

General procedure for $\mathrm{Ni}(\mathrm{cod})_{2} / 2 \mathrm{PPh}_{3}$ catalyzed cyclotrimerization of 6-alkynylpurine 5 with diynes 2.

Into a mixture of $\mathrm{Ni}(\mathrm{cod})_{2}(0.04 \mathrm{mmol}, 11 \mathrm{mg})$ and $\mathrm{PPh}_{3}(0.10 \mathrm{mmol}, 26 \mathrm{mg})$ was added a solution of 6-alkynyl-9-( $\beta$-D-ribofuranosyl)-9H-purine $5^{6}(0.20 \mathrm{mmol})$ and diyne $2(0.22$ mmol) in dry $\mathrm{MeCN}(4 \mathrm{~mL})$. The reaction mixture was initially stirred at $20^{\circ} \mathrm{C}$ for $24 \mathrm{~h}$ or until the consumption of the starting material (TLC). The mixture was evaporated with silica gel and chromatographed on a silica column affording the corresponding 6-aryl-9-( $\beta$-Dribofuranosyl)-9H-purine $\mathbf{4 x y}$.

6-[6-Phenyl-2,2-di(carboxyethyl)indan-5-yl]-9-(ß-D-ribofuranosyl)-9H-purine (4aa).

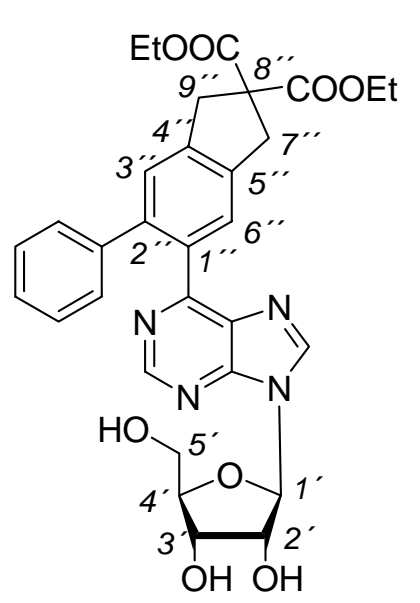

Column chromatography on silica gel (EtOAc) afforded $36 \mathrm{mg}$ $(31 \%)$ of a white foam.

6-[6-Phenyl-2-acetyl-2-(carboxyethyl)indan-5-yl]-9-( $\beta$-D-ribofuranosyl)-9H-purine (4ac).

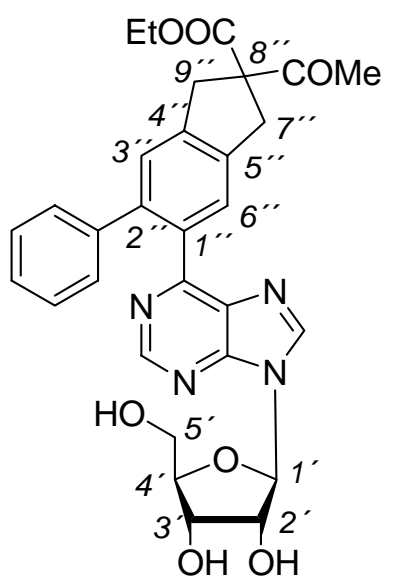

Column chromatography on silica gel (EtOAc/MeOH 10/1) afforded $50 \mathrm{mg}(45 \%)$ of a white foam. 


\section{References.}

1. (a) Kotha, S.; Brahmachary, E. J. Org. Chem. 2000, 65, 1359-1365. (b) Kotha, S.; Mohanraja, K.; Durani, S. Chem. Commun. 2000, 1910-1911. (c) Oediger, H.; Miller, F. Liebigs Ann. Chem. 1976, 348-351.

2. Aresta, M.; Rossi, M.; Sacco, A. Inorg. Chim. Acta 1969, 3, 227-231.

3. Brandsma, L.; Vasilevsky, S. F.; Verkruijsse, H. D. Application of Transition Metal in Organic Synthesis; Springer: Berlin, 1998.

4. Turek, P.; Kotora, M.; Hocek, M.; Votruba, I. Collect. Czech. Chem. Commun. 2005, 70, 339-349.

5. Nauš, P.; Votruba, I.; Hocek, M. Collect. Czech. Chem. Commun. 2004, 69, 1955-1970.

6. Compound $\mathbf{5}$ was prepared essentially as described before: Koyama, S.; Kumazawa, Z.; Kashimura, N. Nucl. Acid Res., Symp. Ser. 1982, 11, 41-44. 
VII. Spectra of the Prepared Compounds.

6-(Hex-1-yn-1-yl)-9-(2,3,5-tri-O-acetyl- $\beta$-D-ribofuranosyl)-9H-purine (1b).

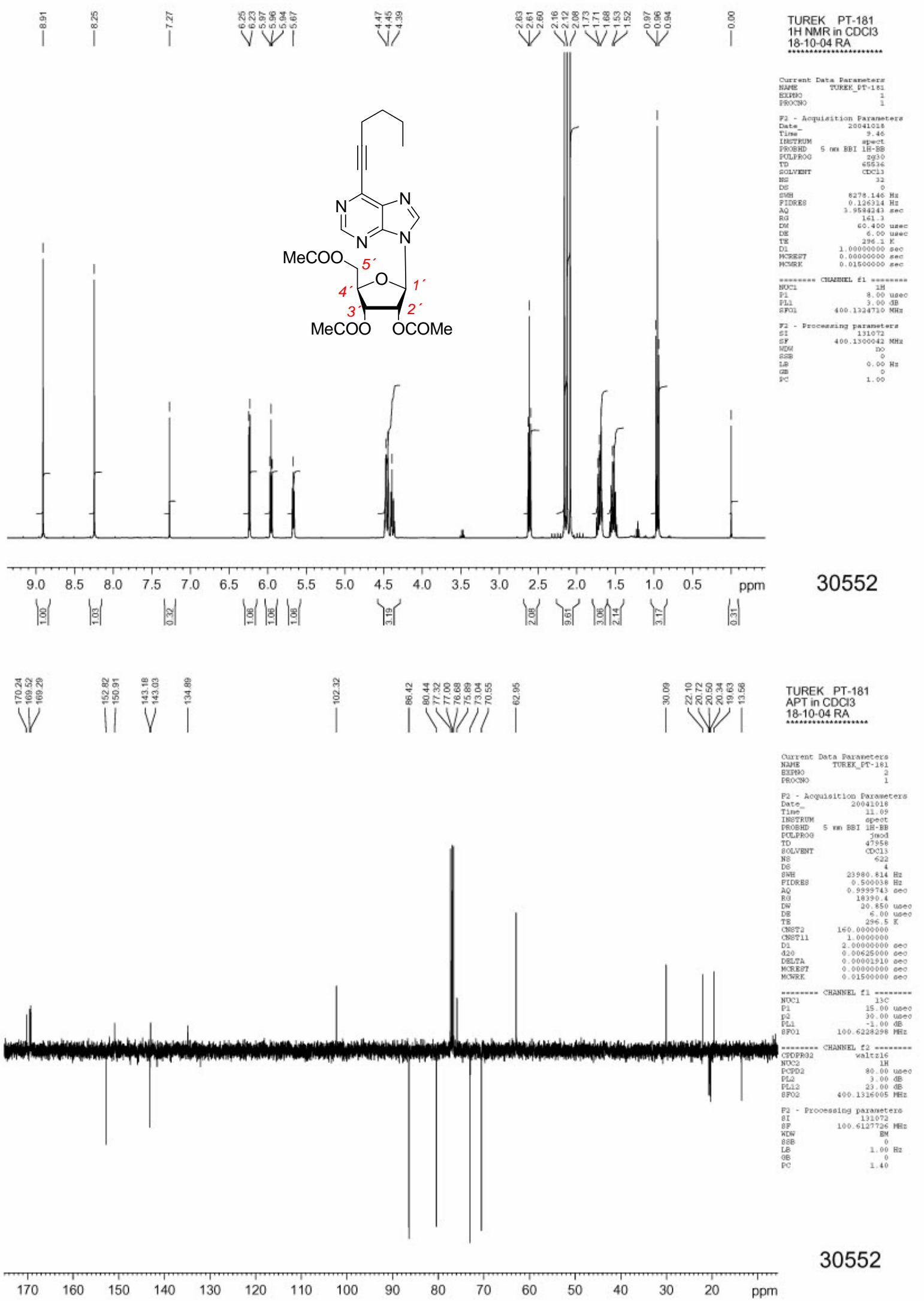


6-[6-Phenyl-2,2-di(carboxyethyl)indan-5-yl]-9-(2,3,5-tri-O-acetyl- $\beta$-D-ribofuranosyl)-9Hpurine (3aa).
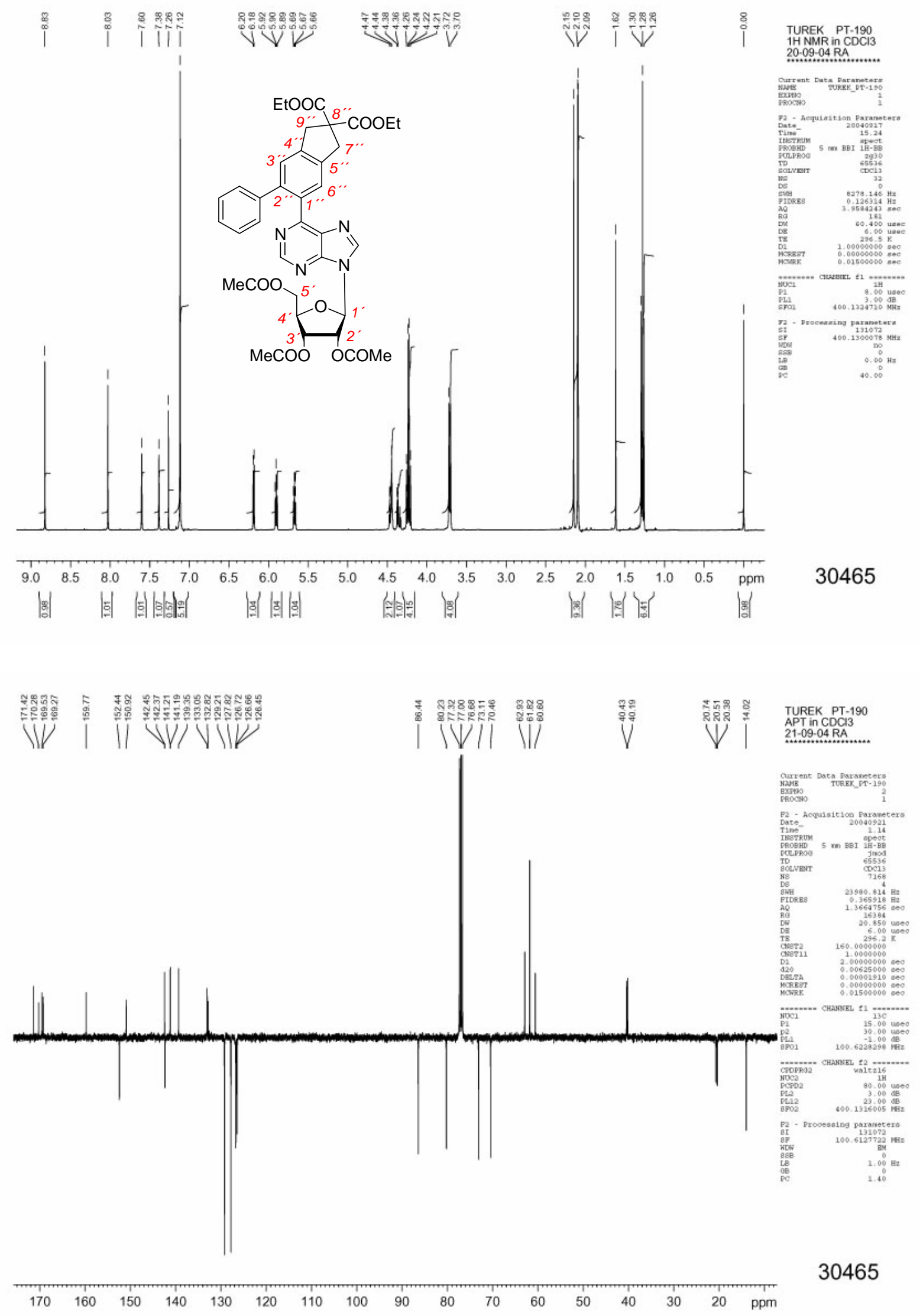
6-[6-Phenyl-(2,2-diacetylindan-5-yl)]-9-(2,3,5-tri-O-acetyl- $\beta$-D-ribofuranosyl)-9H-purine (3ab).
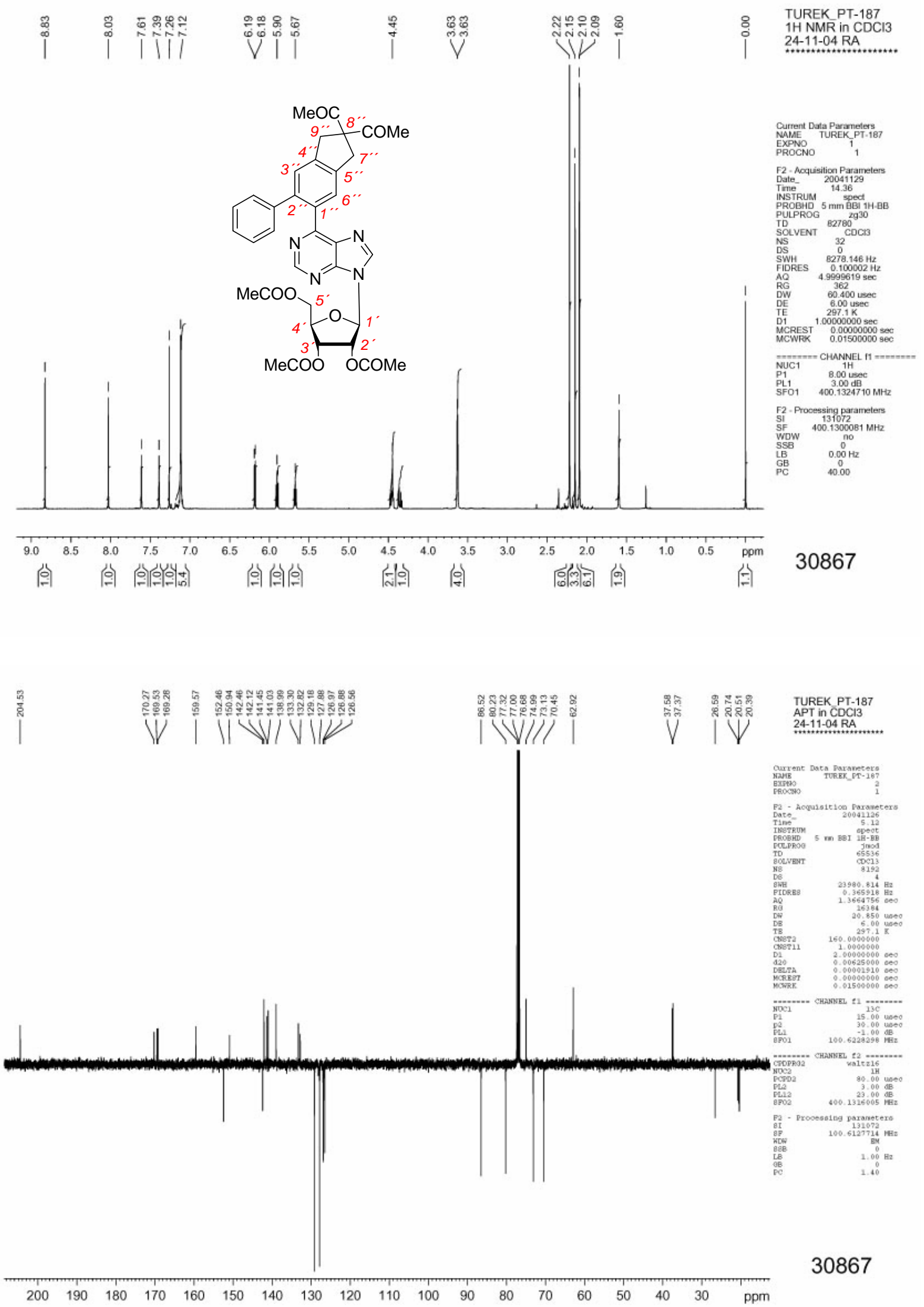
6-[6-Phenyl-2-acetyl-2-(carboxyethyl)indan-5-yl]-9-(2,3,5-tri-O-acetyl- $\beta$-D-ribofuranosyl) $-9 H$-purine (3ac).
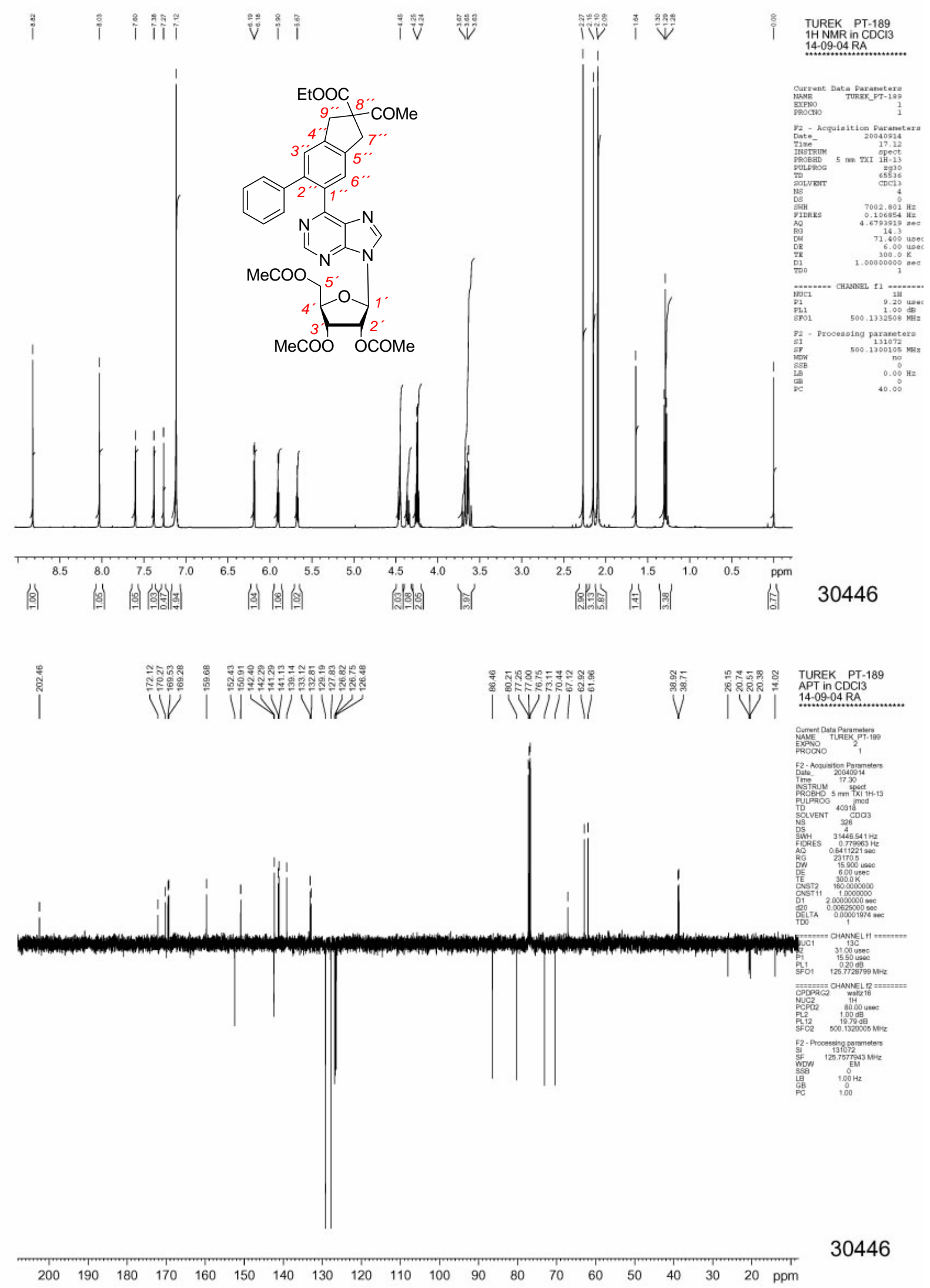
6-[6-Phenyl-2-cyano-2-(carboxyethyl)indan-5-yl]-9-(2,3,5-tri-O-acetyl- $\beta$-D-ribofuranosyl) $-9 H$-purine (3ad).
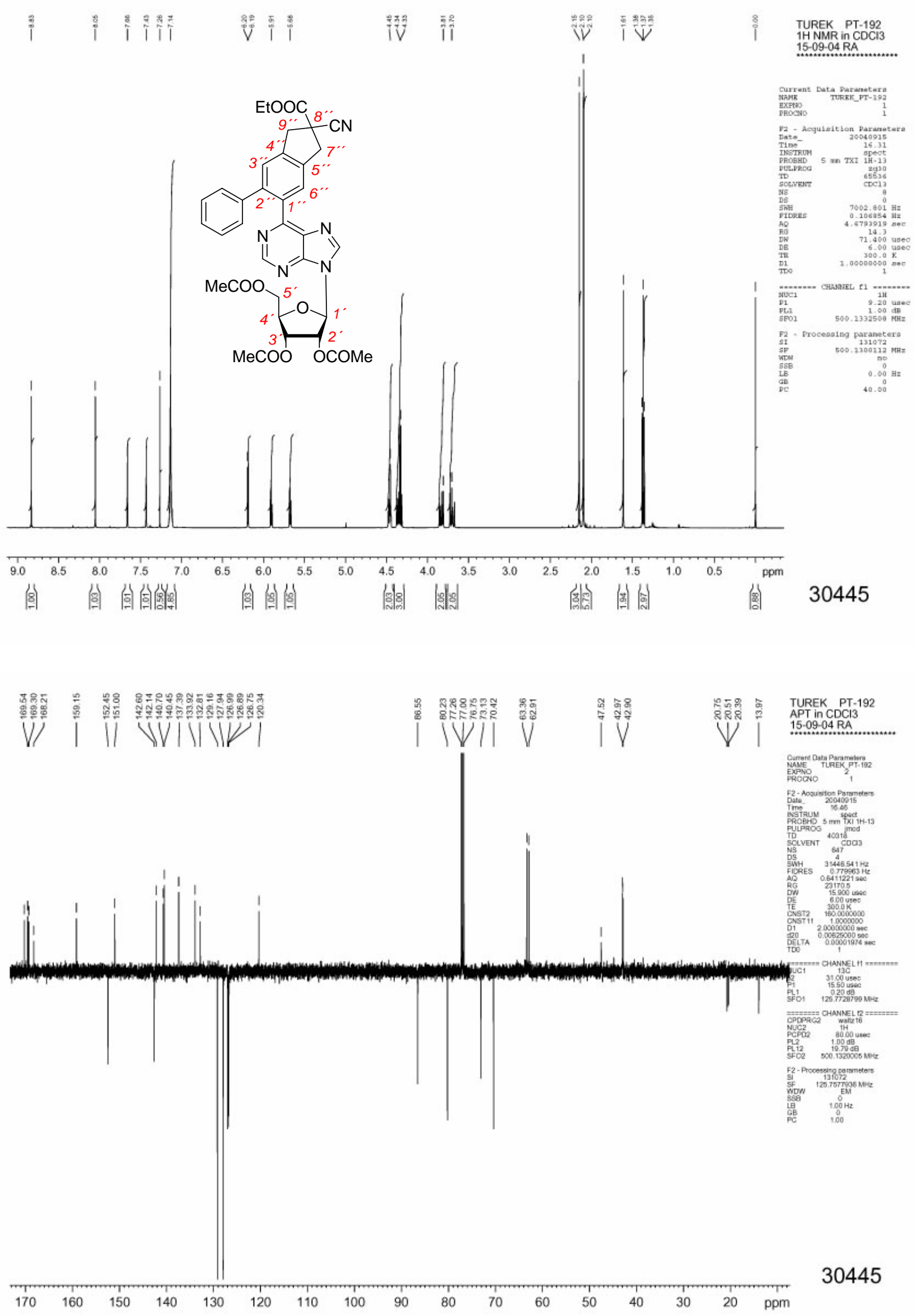
6-[6-Phenyl-1,3-dihydro-2-tosyl-2H-indol-5-yl]-9-(2,3,5-tri-O-acetyl- $\beta$-D-ribofuranosyl)$9 H$-purine (3ae).
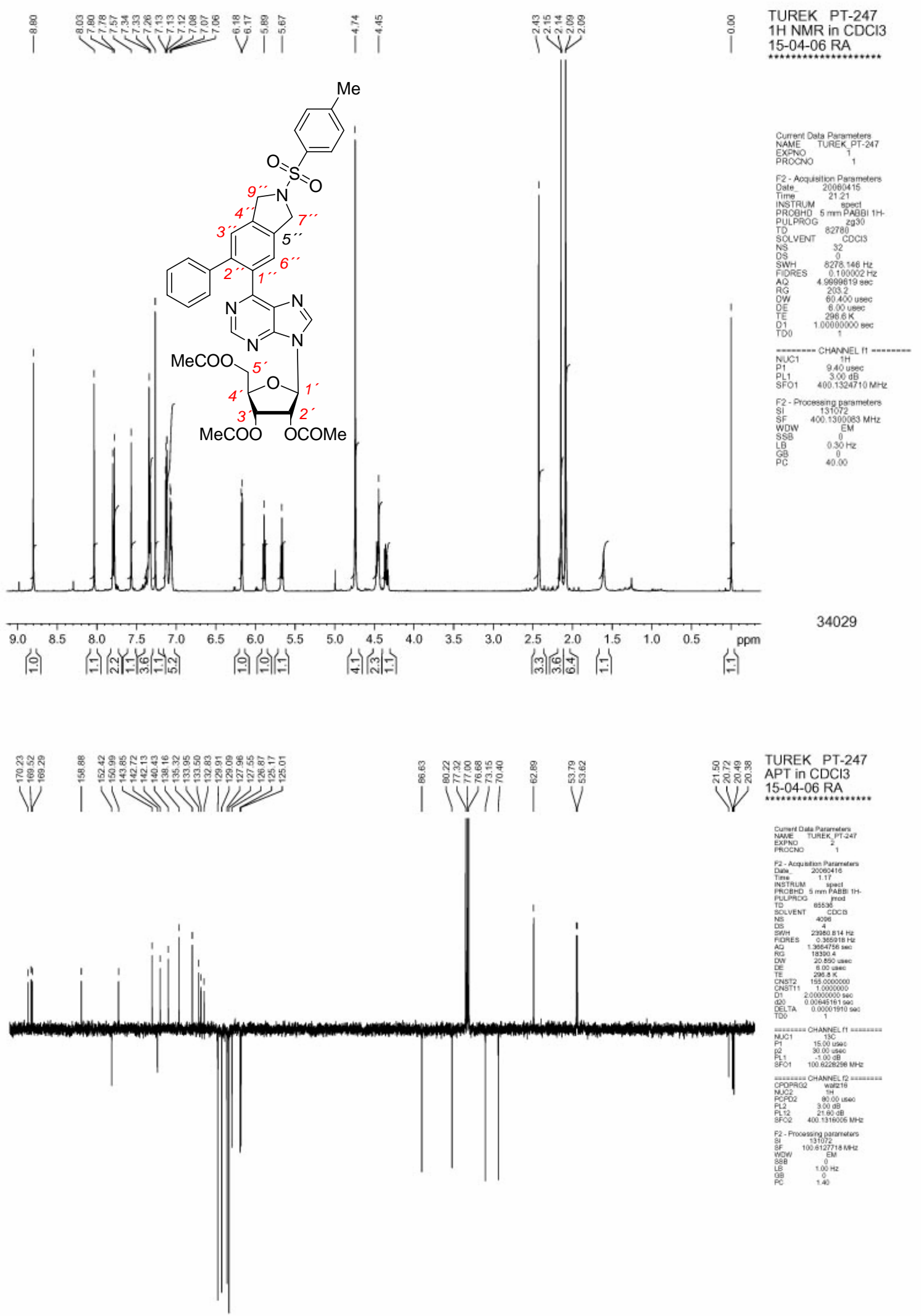

TUREK PT-247

APT in $\mathrm{CDCl} 3$

15-04-06 RA

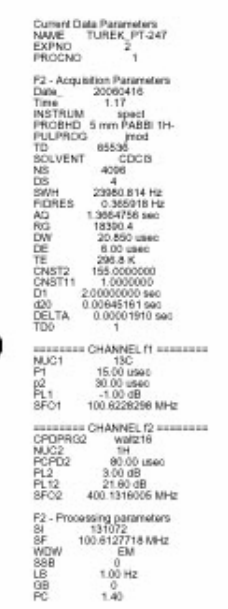

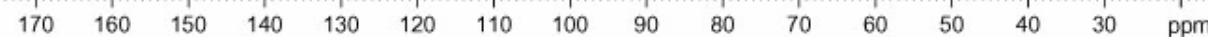


6-[6-Phenyl-1,3-dihydro-2-phenyl-2H-indol-5-yl]-9-(2,3,5-tri-O-acetyl- $\beta$-D-ribofuranosyl) $-9 H$-purine (3af).
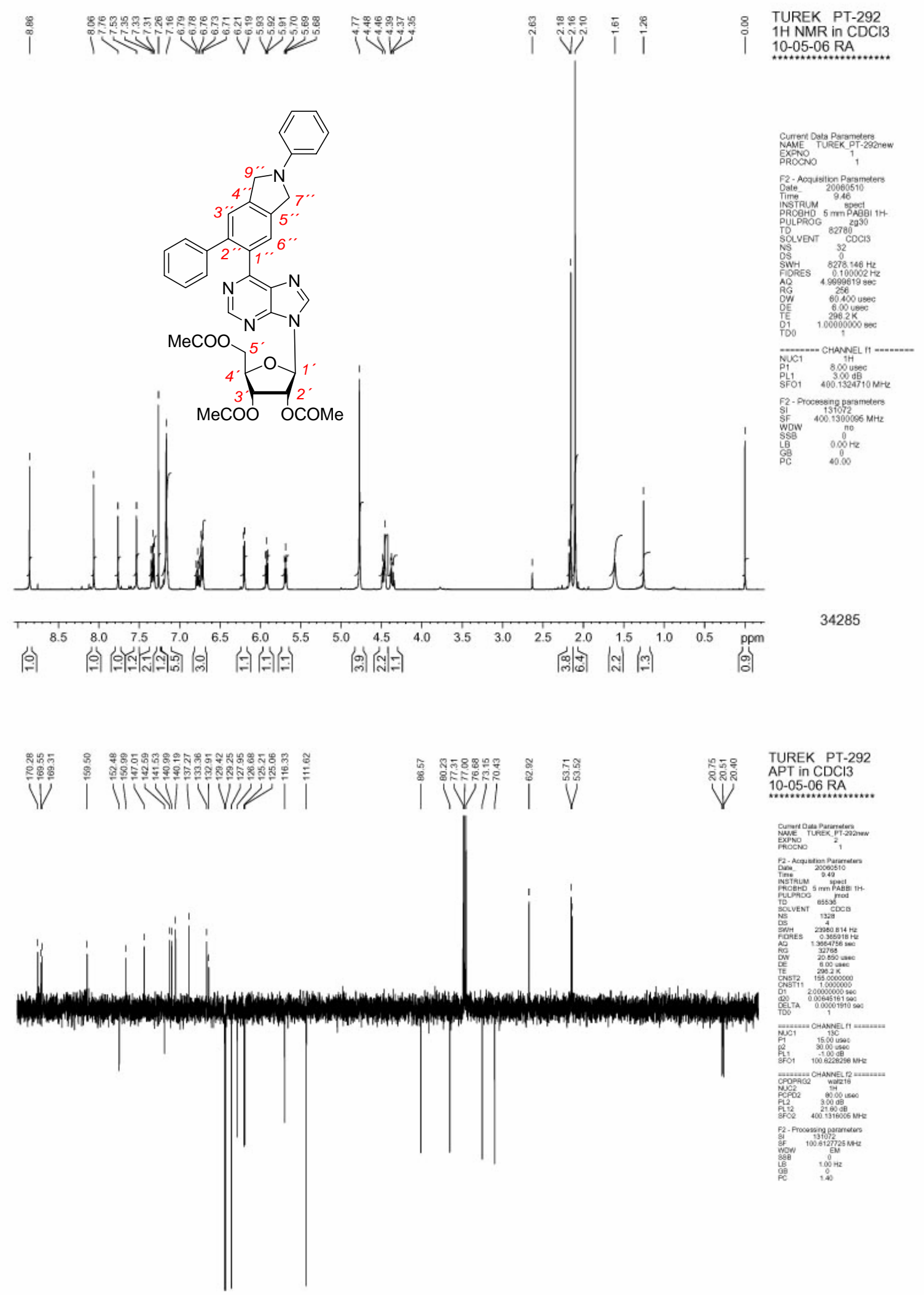

TUREK PT-292

APT in $\mathrm{CDCl} 3$

10-05-06 RA.

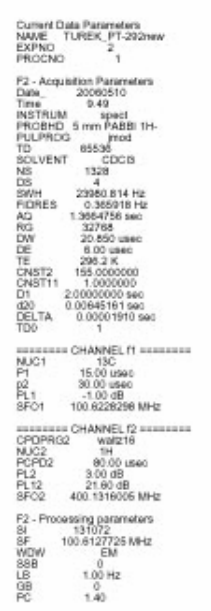

$\begin{array}{lllllllllllllll}170 & 160 & 150 & 140 & 130 & 120 & 110 & 100 & 90 & 80 & 70 & 60 & 50 & 40 & 30\end{array}$ 
6-[6-Phenyl-1,3-dihydroisobenzofuran-5-yl)]-9-(2,3,5-tri-O-acetyl- $\beta$-D-ribofuranosyl)$9 H$-purine (3ag).
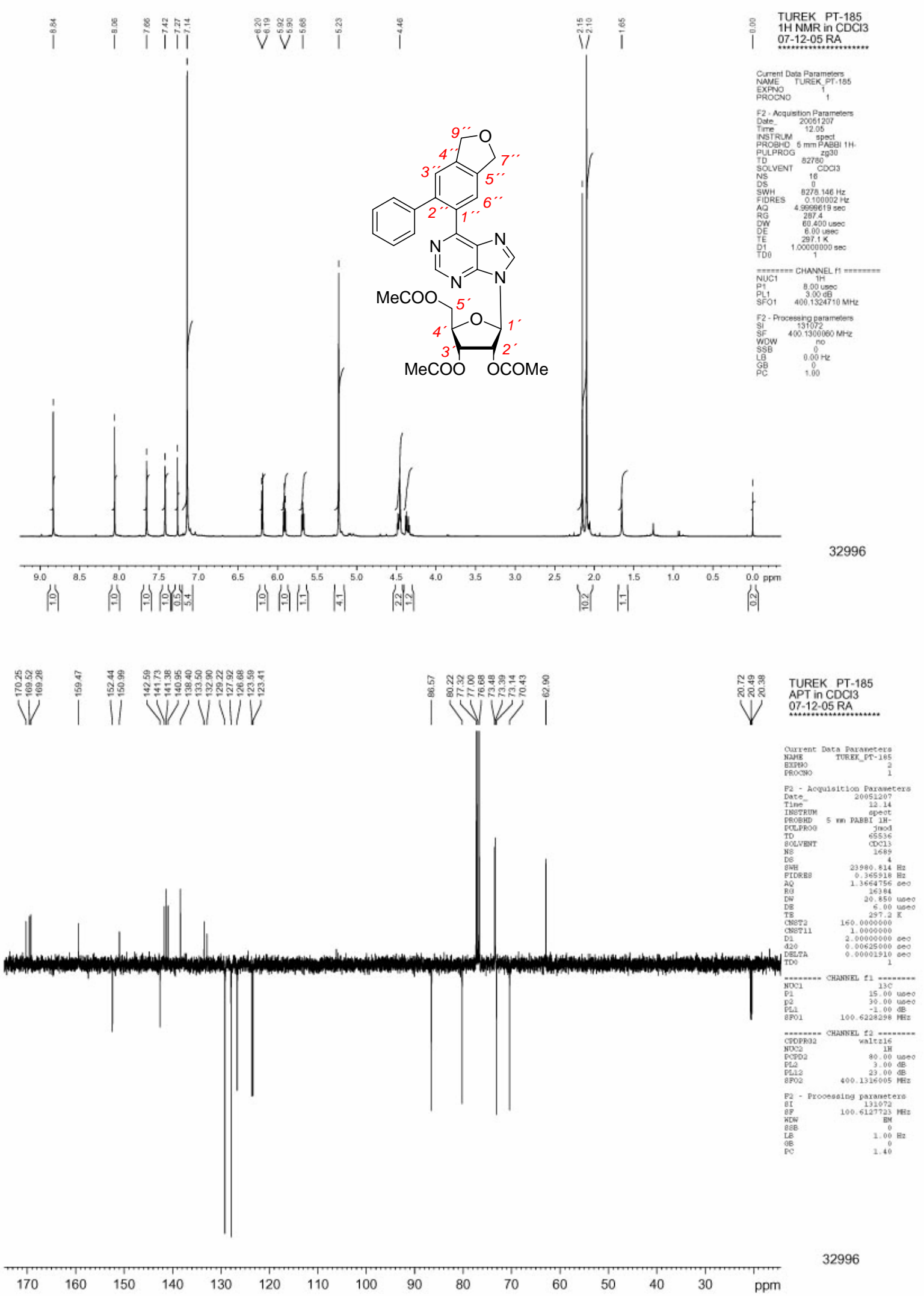
6-[6-Phenyl-2-[[(1,1-dimethylethoxy)carbonyl]amino]-2-(carboxyethyl)indan-5-yl)]-9$(2,3,5$-tri-O-acetyl- $\beta$-D-ribofuranosyl)-9H-purine (3ah).
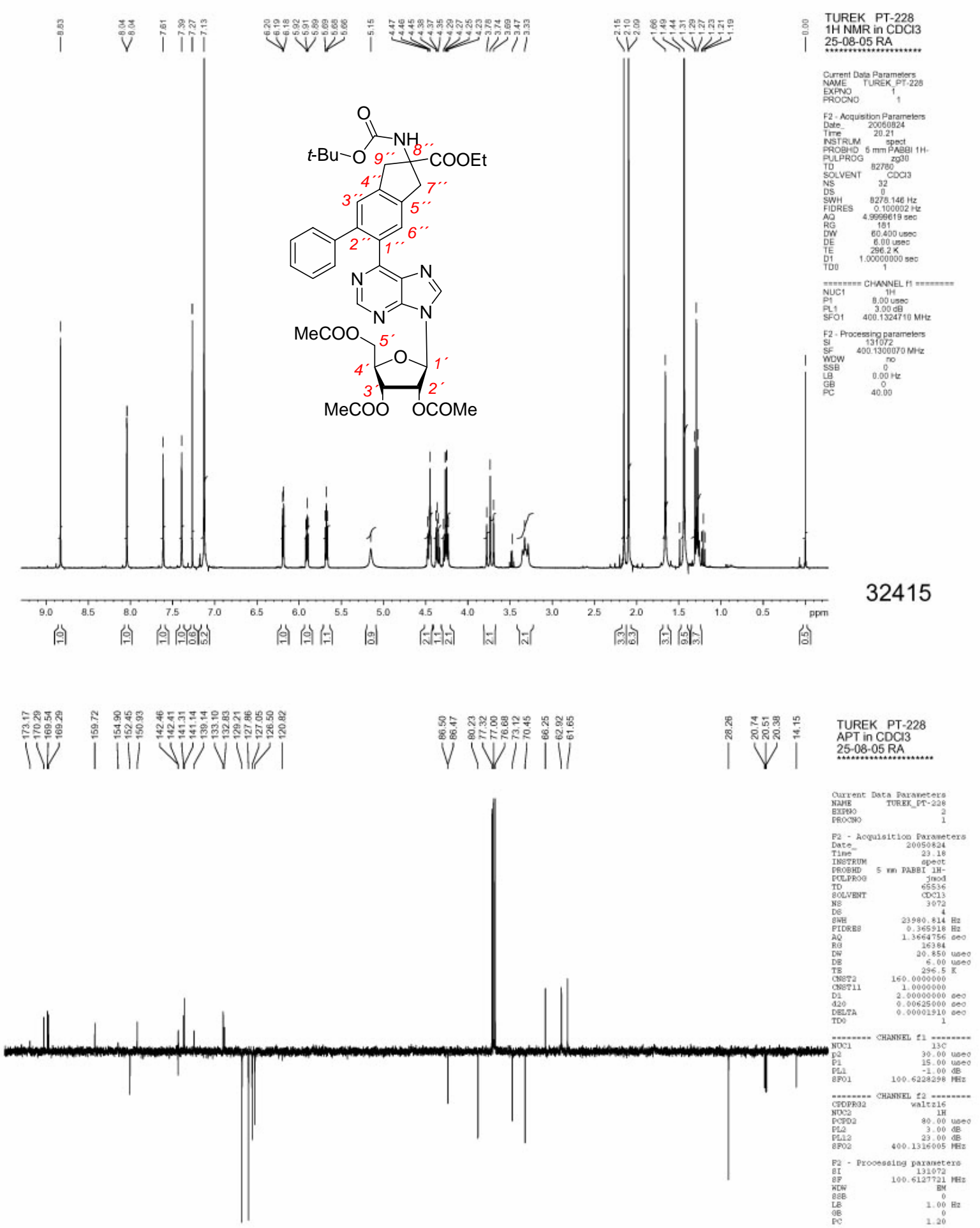
6-[3-Phenyl-5,6,7,8-tetrahydronaphthalen-2-yl)]-9-(2,3,5-tri-O-acetyl- $\beta$-D-ribofuranosyl)$9 H$-purine (3ai).

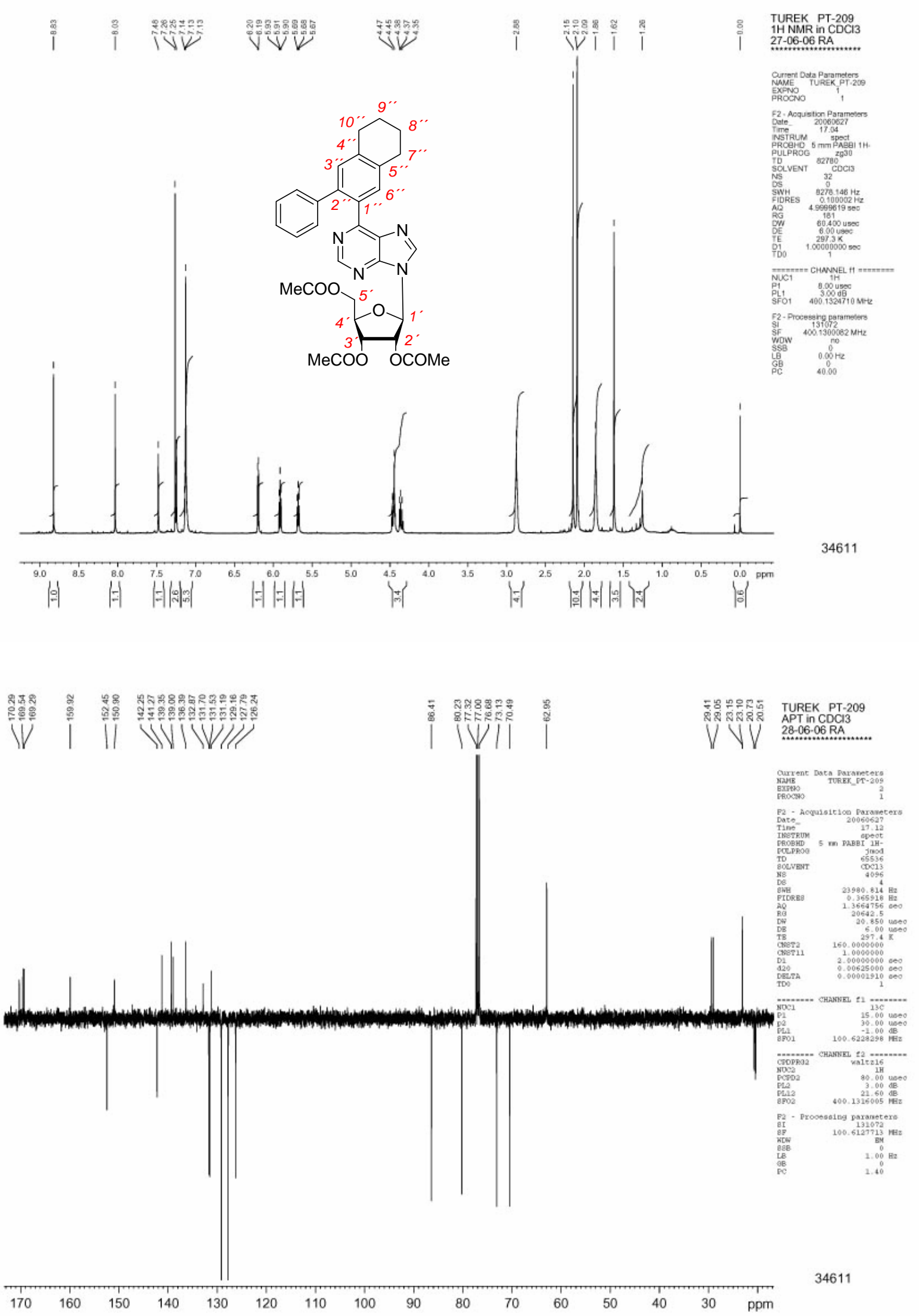


6-[6-Butyl-2,2-di(carboxyethyl)indan-5-yl]-9-(2,3,5-tri-O-acetyl- $\beta$-D-ribofuranosyl)-9Hpurine (3ba).
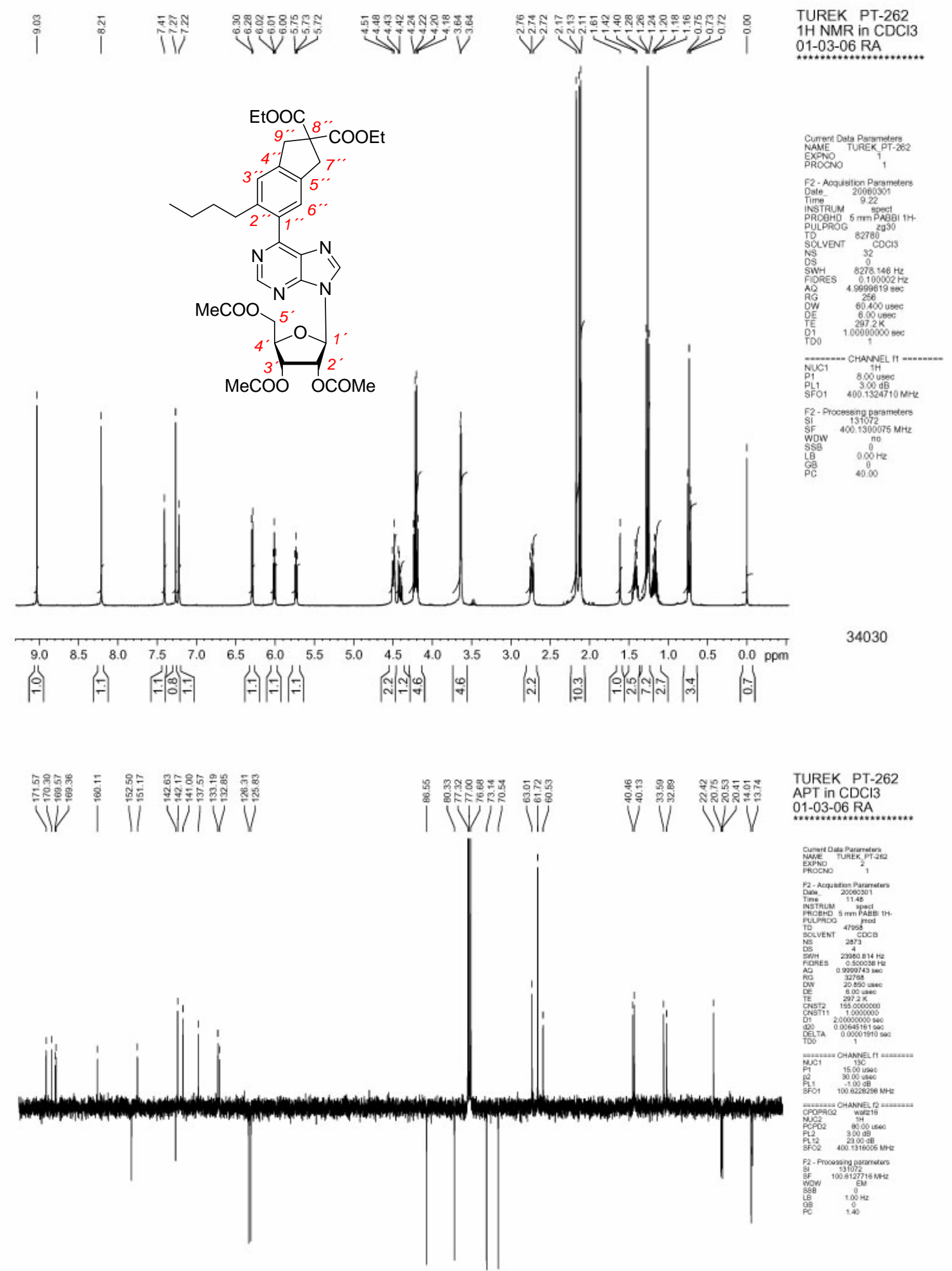

TUREK PT-262

01-03-06 RA

N.......

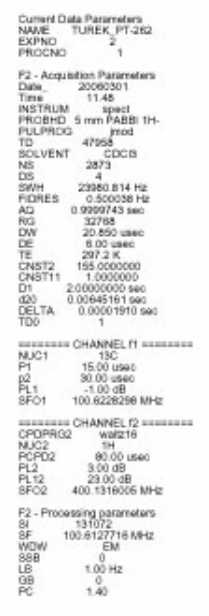


6-[6-Butyl-2-cyano-2-(carboxyethyl)indan-5-yl]-9-(2,3,5-tri-O-acetyl- $\beta$-D-ribofuranosyl)$9 H$-purine (3bd).
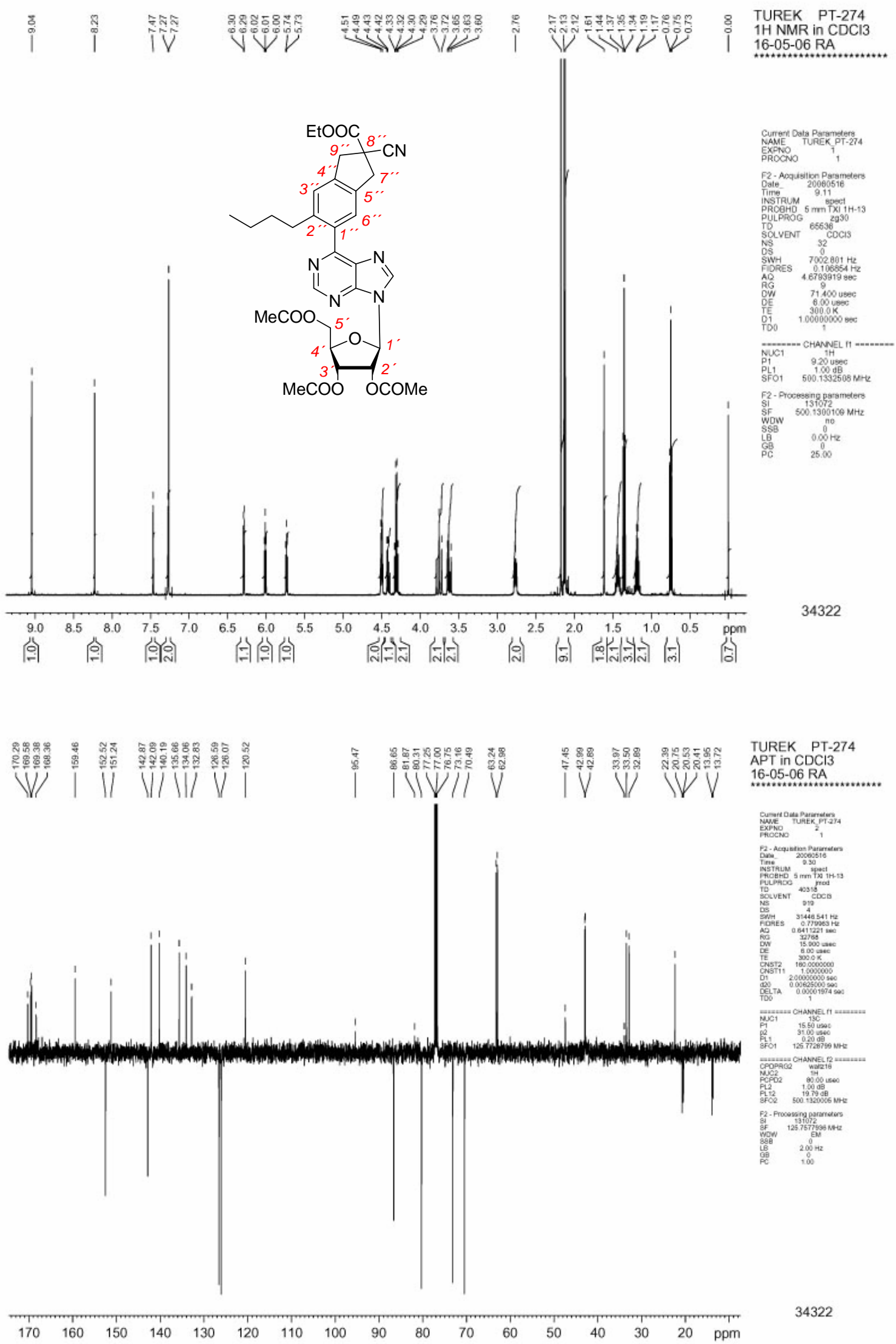
6-[6-Butyl-1,3-dihydroisobenzofuran-5-yl]-9-(2,3,5-tri-O-acetyl- $\beta$-D-ribofuranosyl)-9Hpurine (3bg).
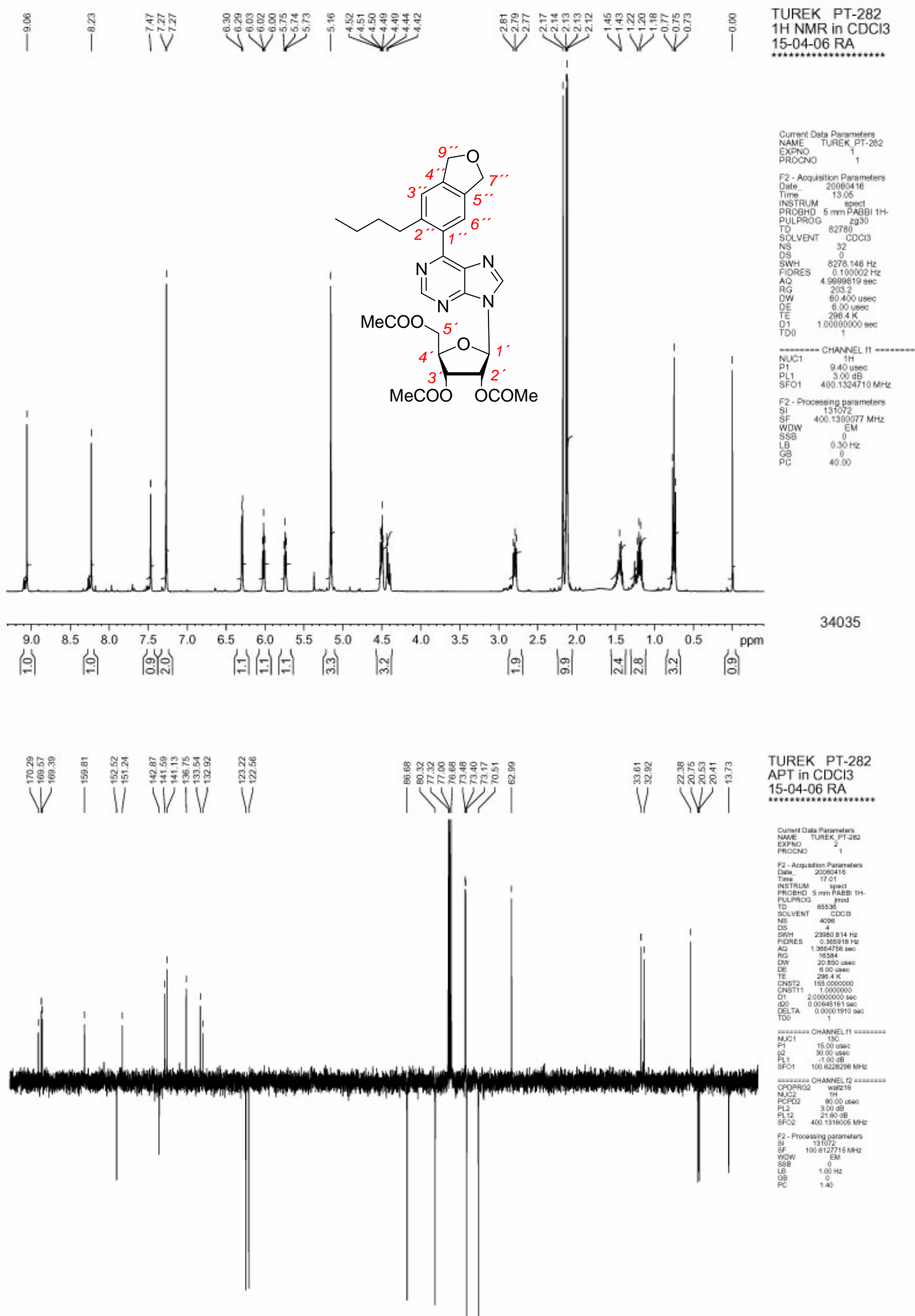

TUREK PT-282

APT in $\mathrm{CDCl} 3$

15-04-06 RA

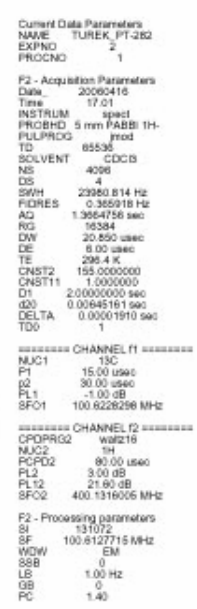

$\begin{array}{lllllllllllllllll}170 & 160 & 150 & 140 & 130 & 120 & 110 & 100 & 90 & 80 & 70 & 60 & 50 & 40 & 30 & 20 & \text { ppm }\end{array}$ 
6-[6-Trimethylsilyl-2,2-di(carboxyethyl)indan-5-yl]-9-(2,3,5-tri-O-acetyl- $\beta$-D-ribofuranosyl)-9H-purine (3ca).
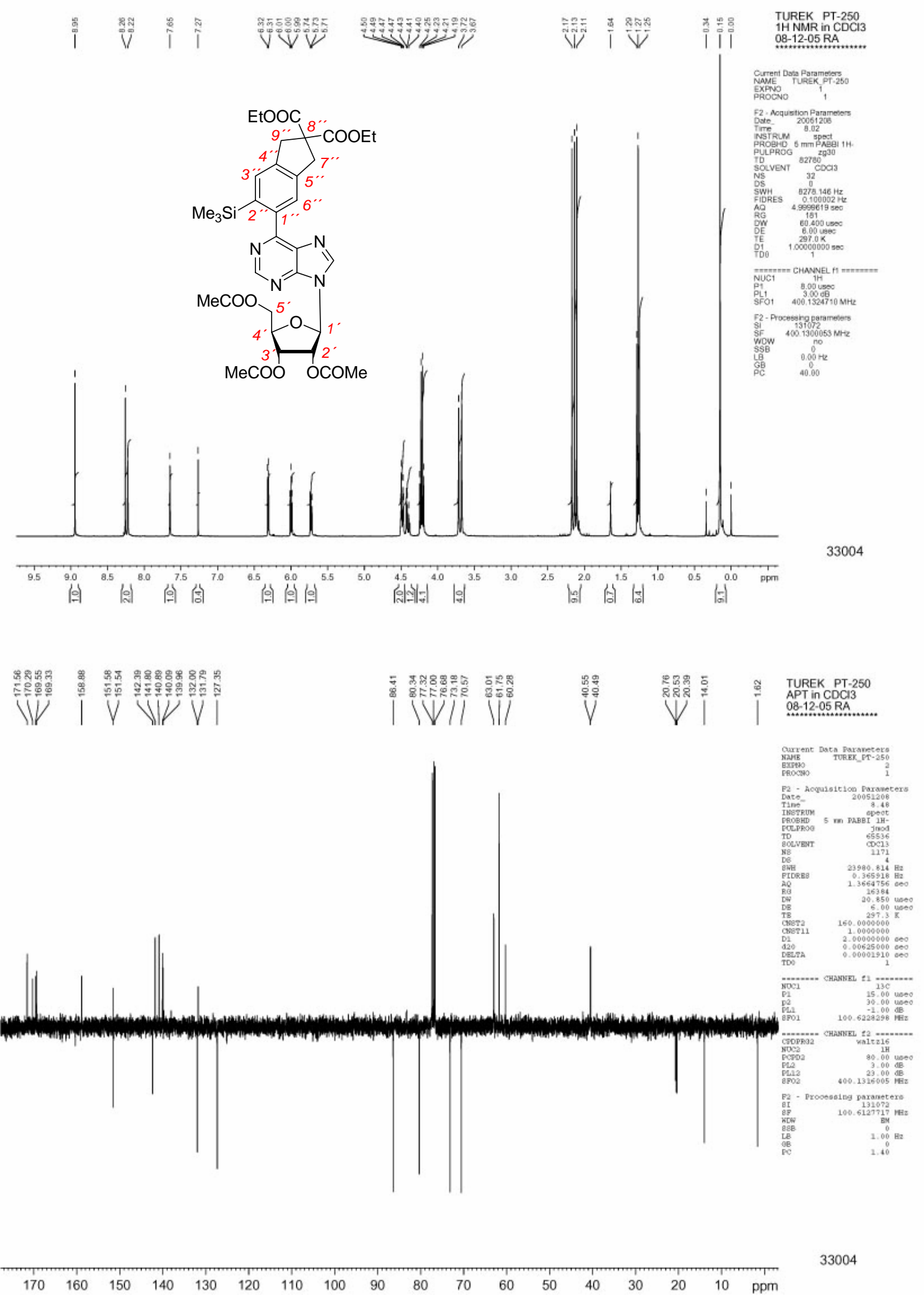
6-[6-Trimethylsilyl-1,3-dihydro-2-tosyl-2H-indol-5-yl]-9-(2,3,5-tri-O-acetyl- $\beta$-D-ribofuranosyl)-9H-purine (3ce).

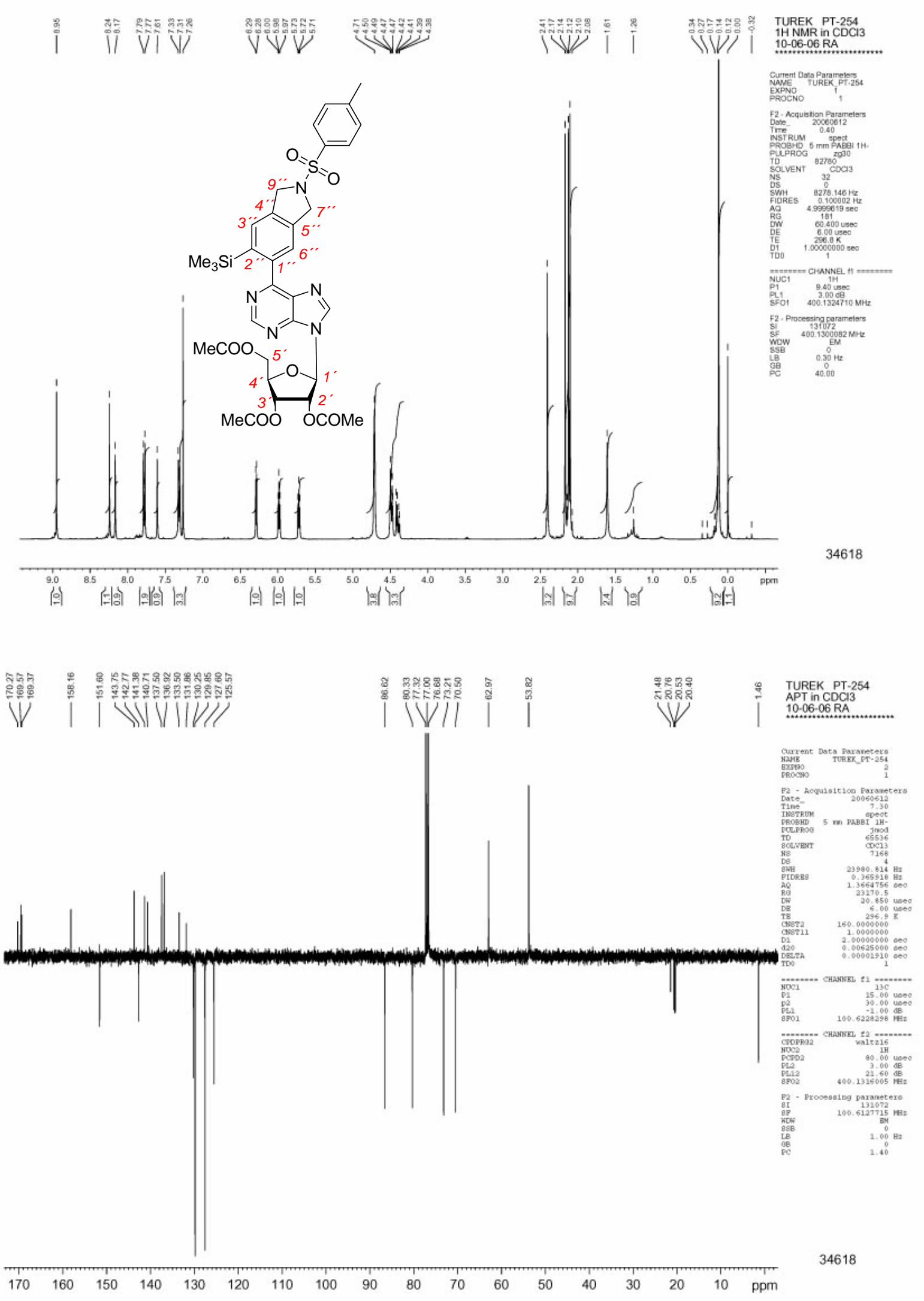


6-[6-Trimethylsilyl-1,3-dihydroisobenzofuran-5-yl]-9-(2,3,5-tri-O-acetyl- $\beta$-D-ribofuranosyl)-9H-purine (3cg).
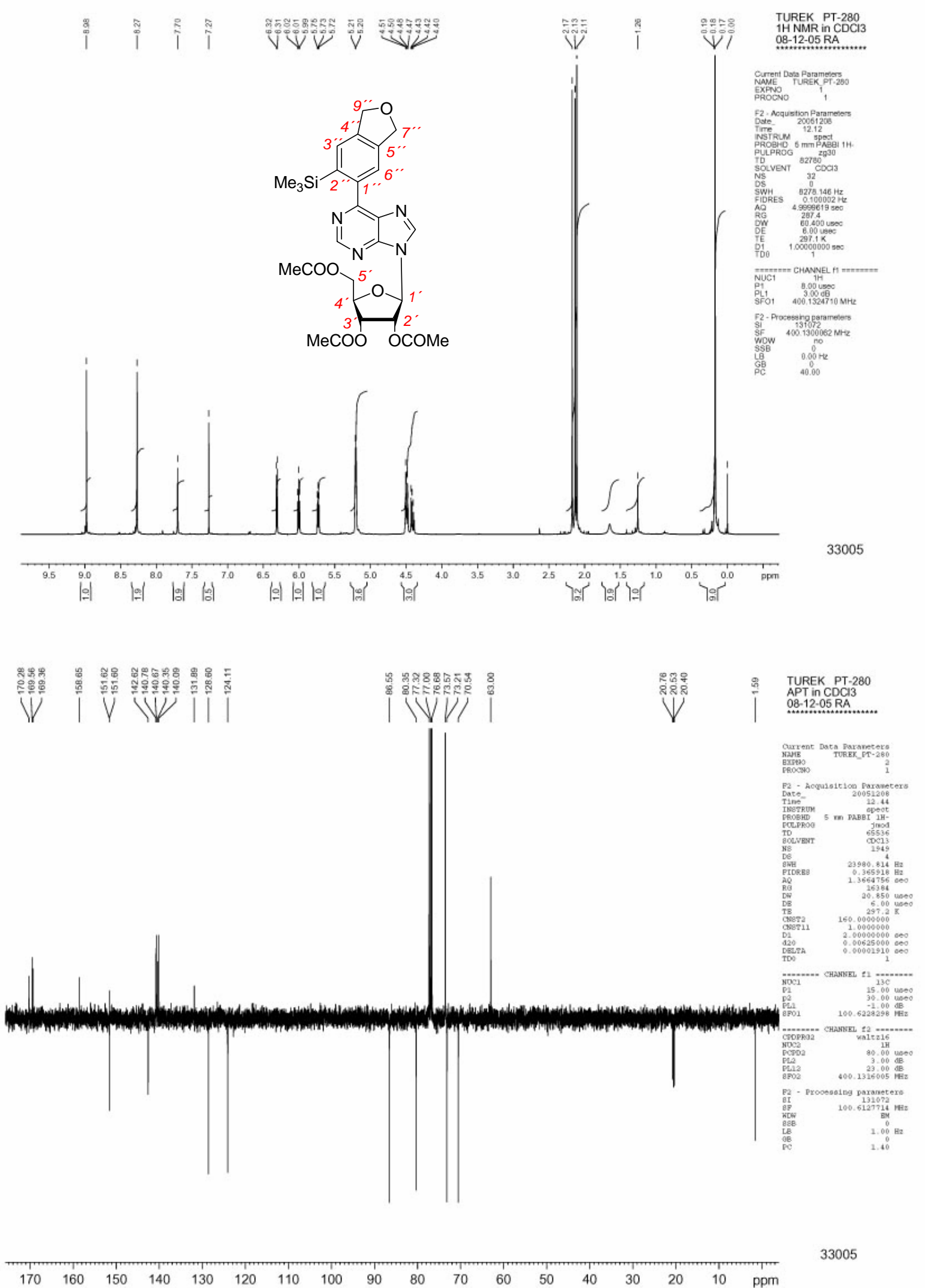
6-[6-Phenyl-2,2-di(carboxyethyl)indan-5-yl]-9-( $\beta$-D-ribofuranosyl)-9H-purine (4aa).
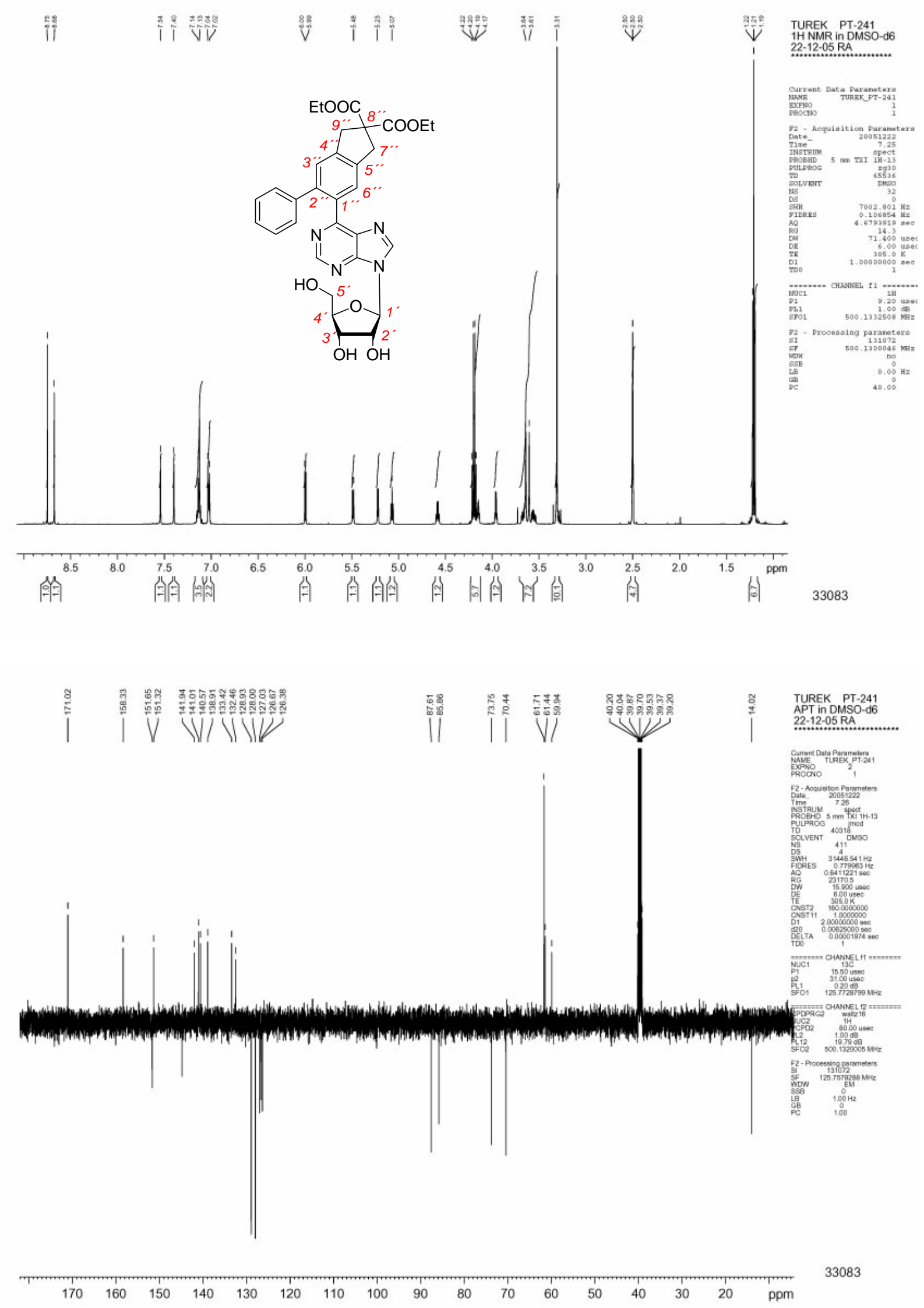
6-[6-Phenyl-2-acetyl-2-(carboxyethyl)indan-5-yl]-9-( $\beta$-D-ribofuranosyl)-9H-purine (4ac).
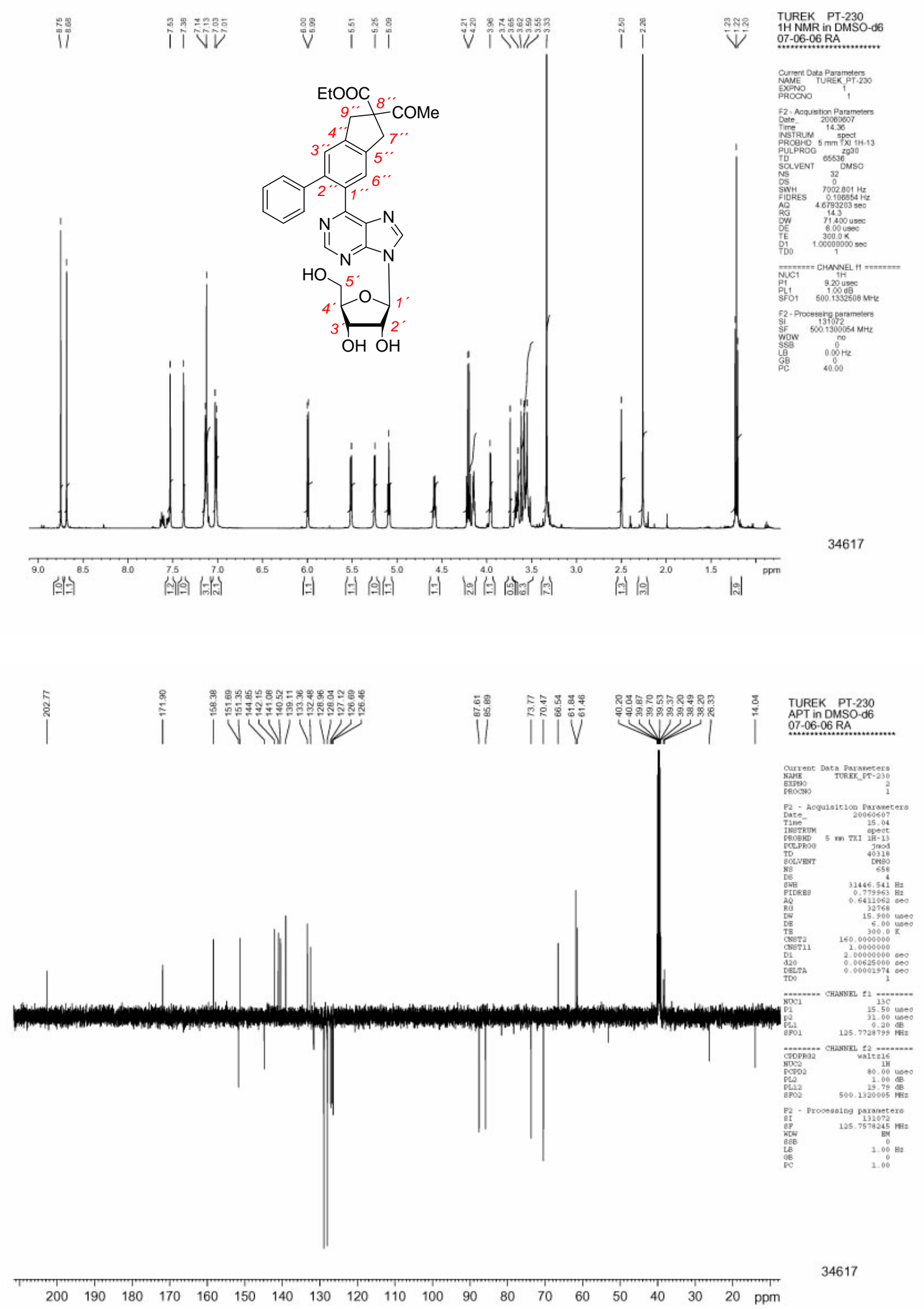
6-[6-Phenyl-2-cyano-2-(carboxymethyl)indan-5-yl]-9-( $\beta$-D-ribofuranosyl)-9H-purine (4ad).
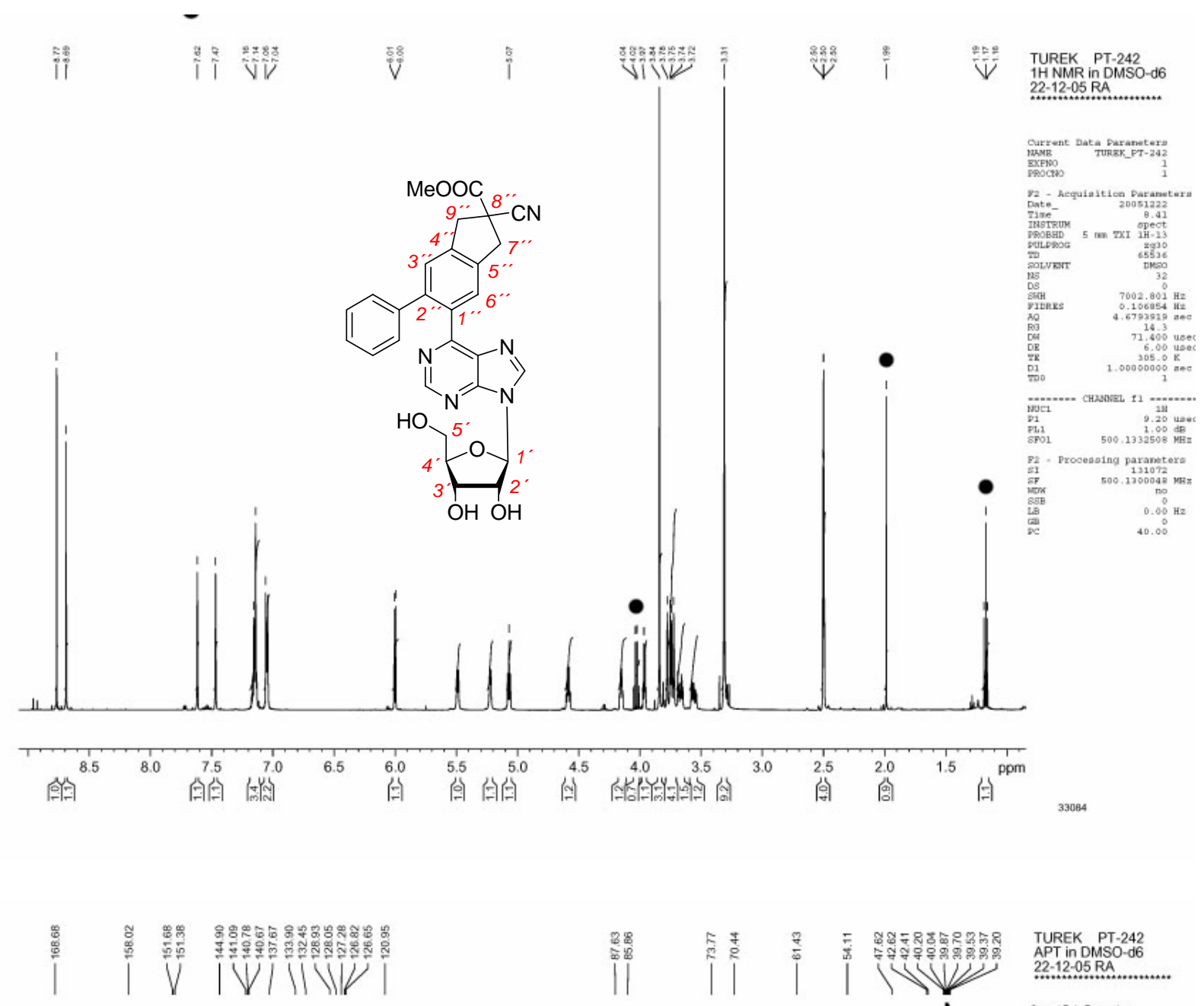

TUREK PT-242 APT in DMSO-d6 22-12-05 RA.
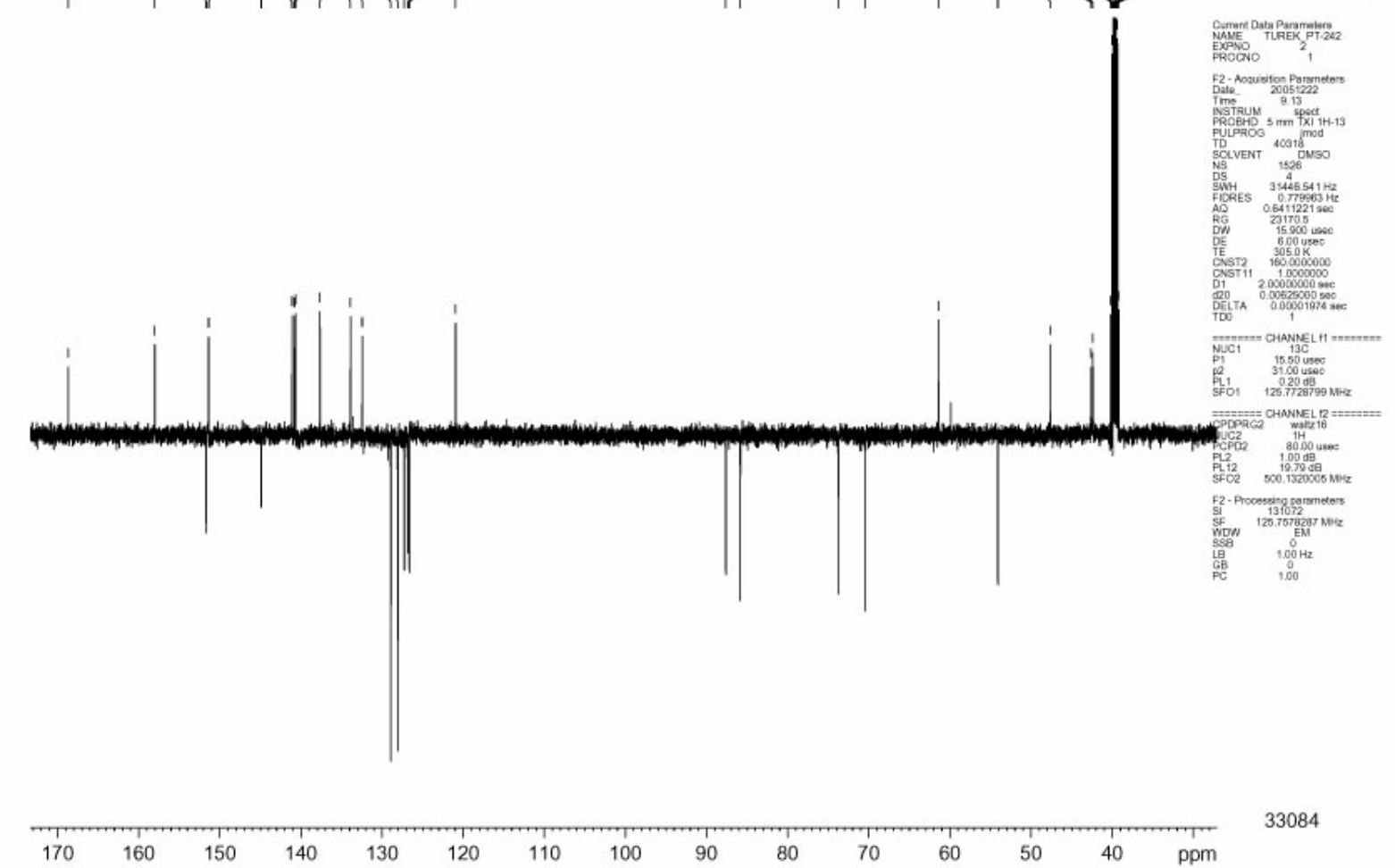
6-[6-Phenyl-1,3-dihydro-2-tosyl-2H-indol-5-yl)]-9-(ß-D-ribofuranosyl)-9H-purine (4ae).
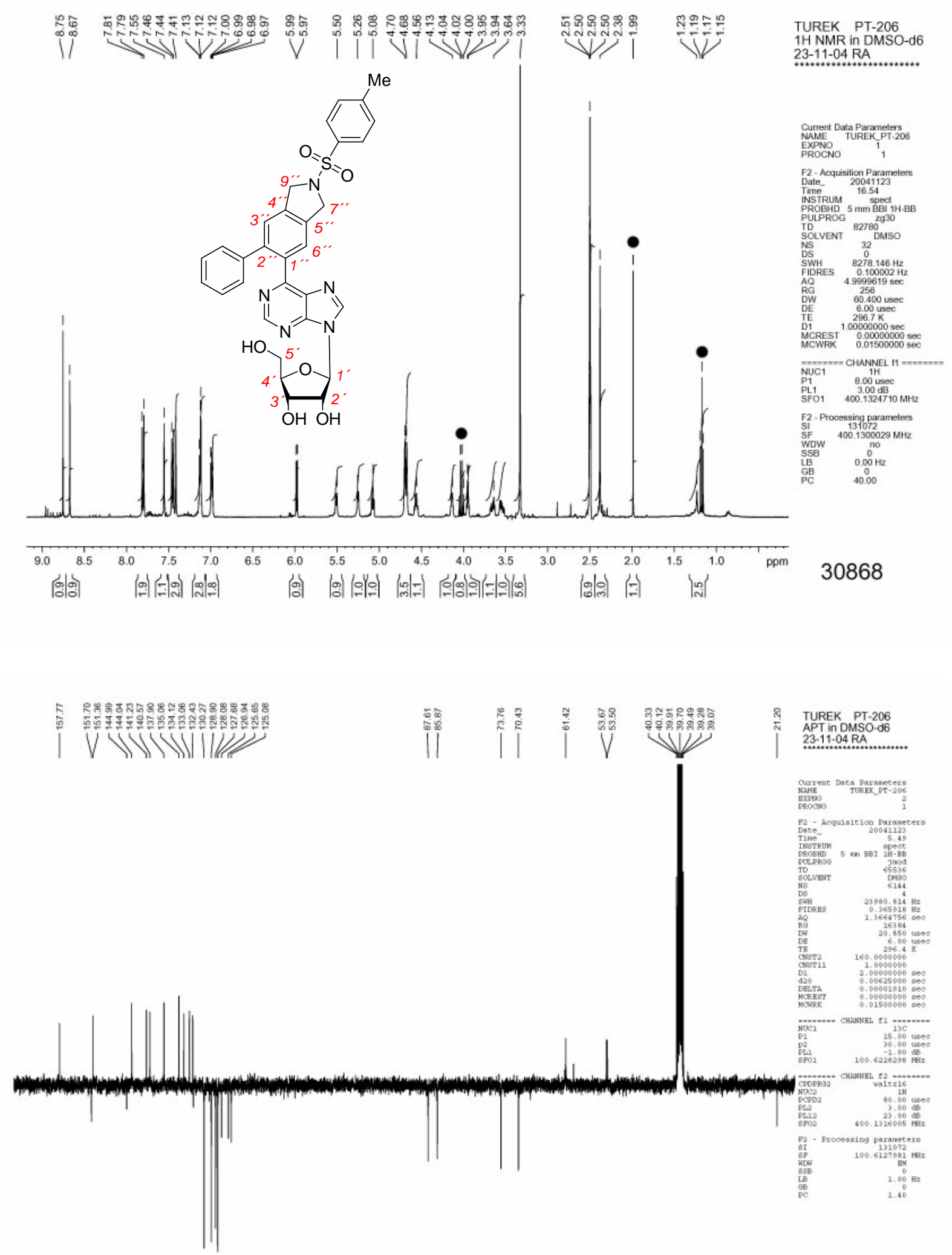

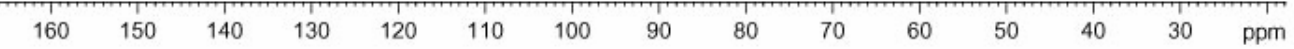


6-[6-Phenyl-1,3-dihydroisobenzofuran-5-yl]-9-( $\beta$-D-ribofuranosyl)-9H-purine (4ag).
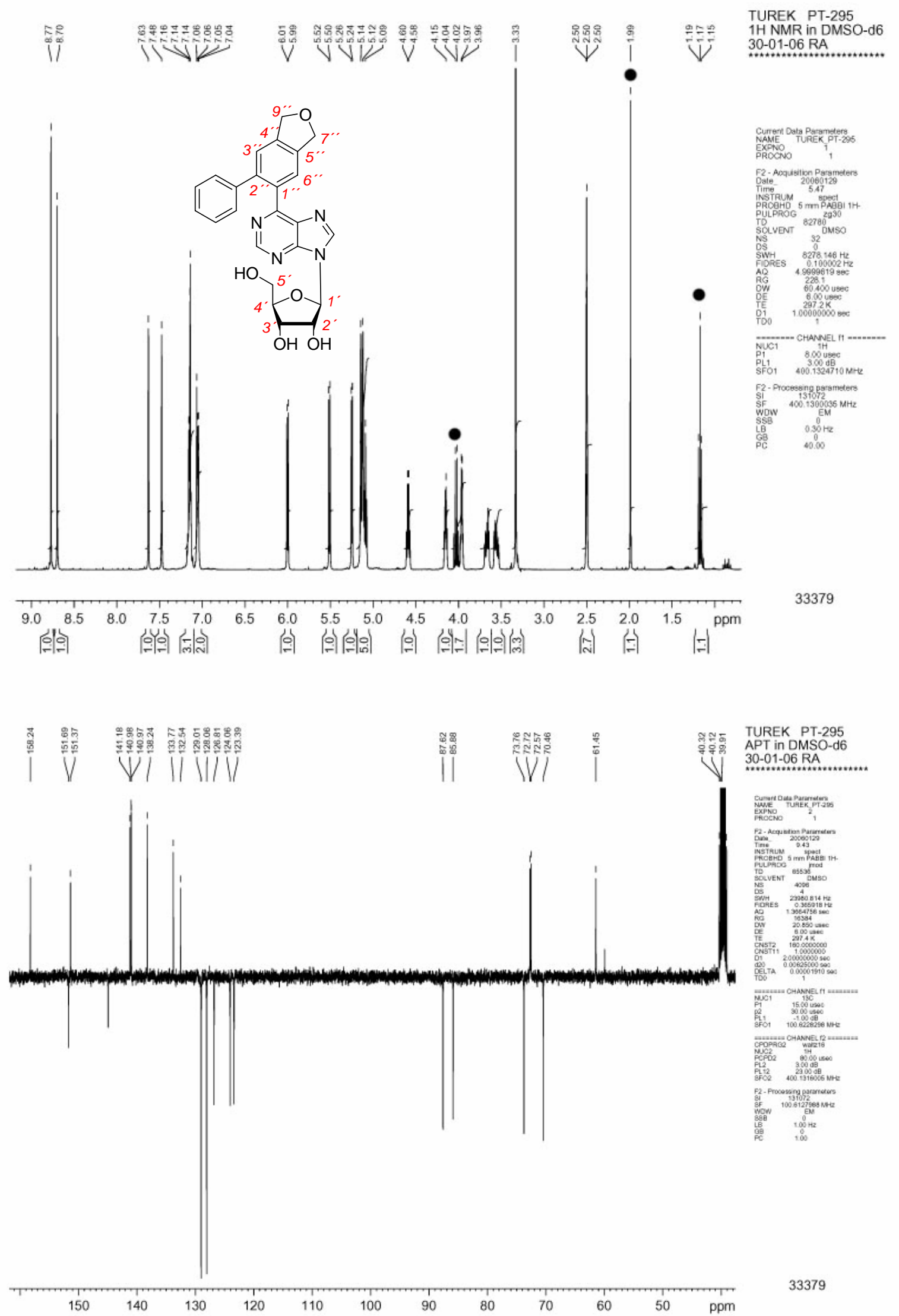
6-[6-Butyl-2,2-di(carboxyethyl)indan-5-yl]-9-( $\beta$-D-ribofuranosyl)-9H-purine (4ba).
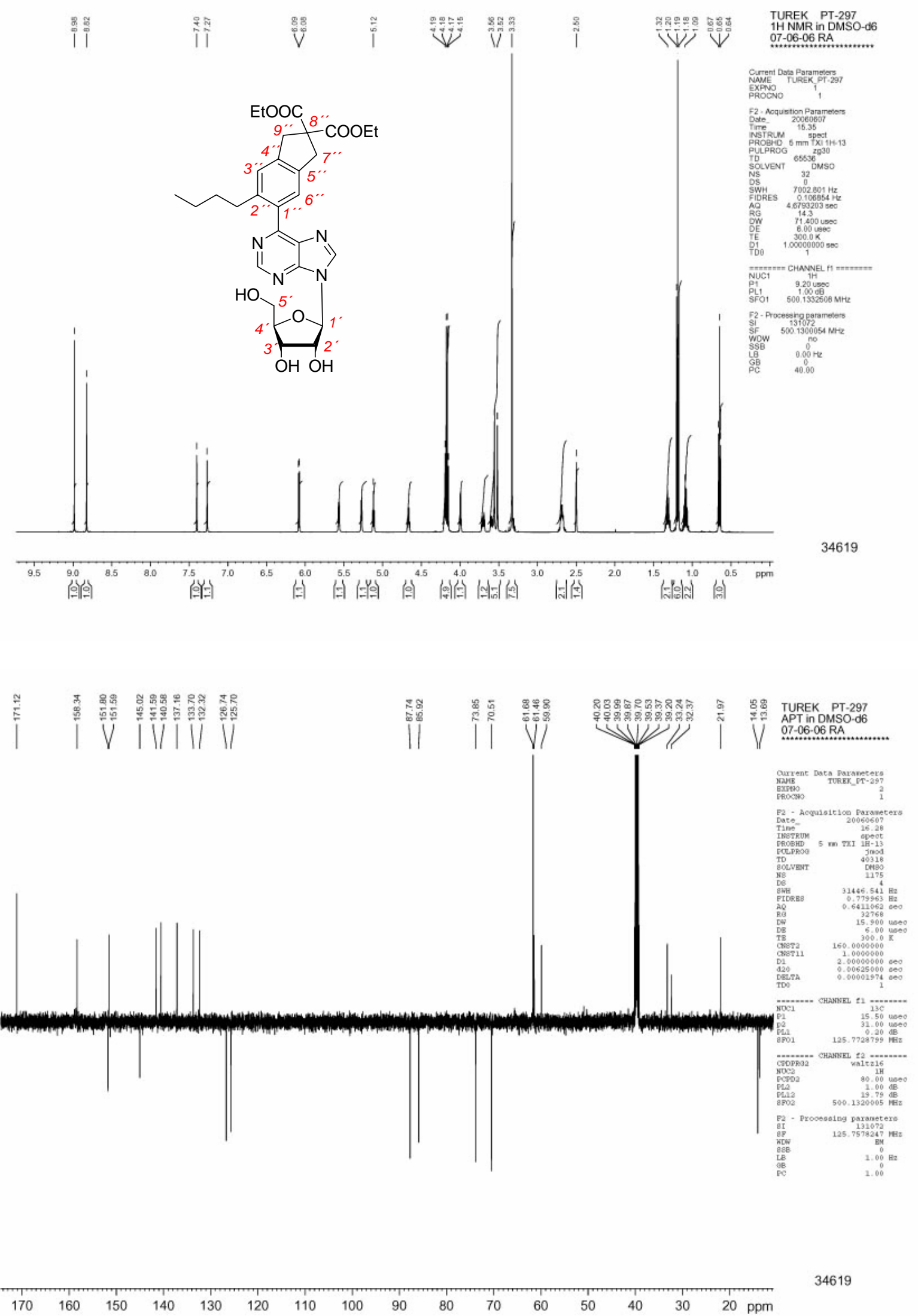
6-[6-Trimethylsilyl-2,2-di(carboxyethyl)indan-5-yl]-9-(ß-D-ribofuranosyl)-9H-purine (4ca).
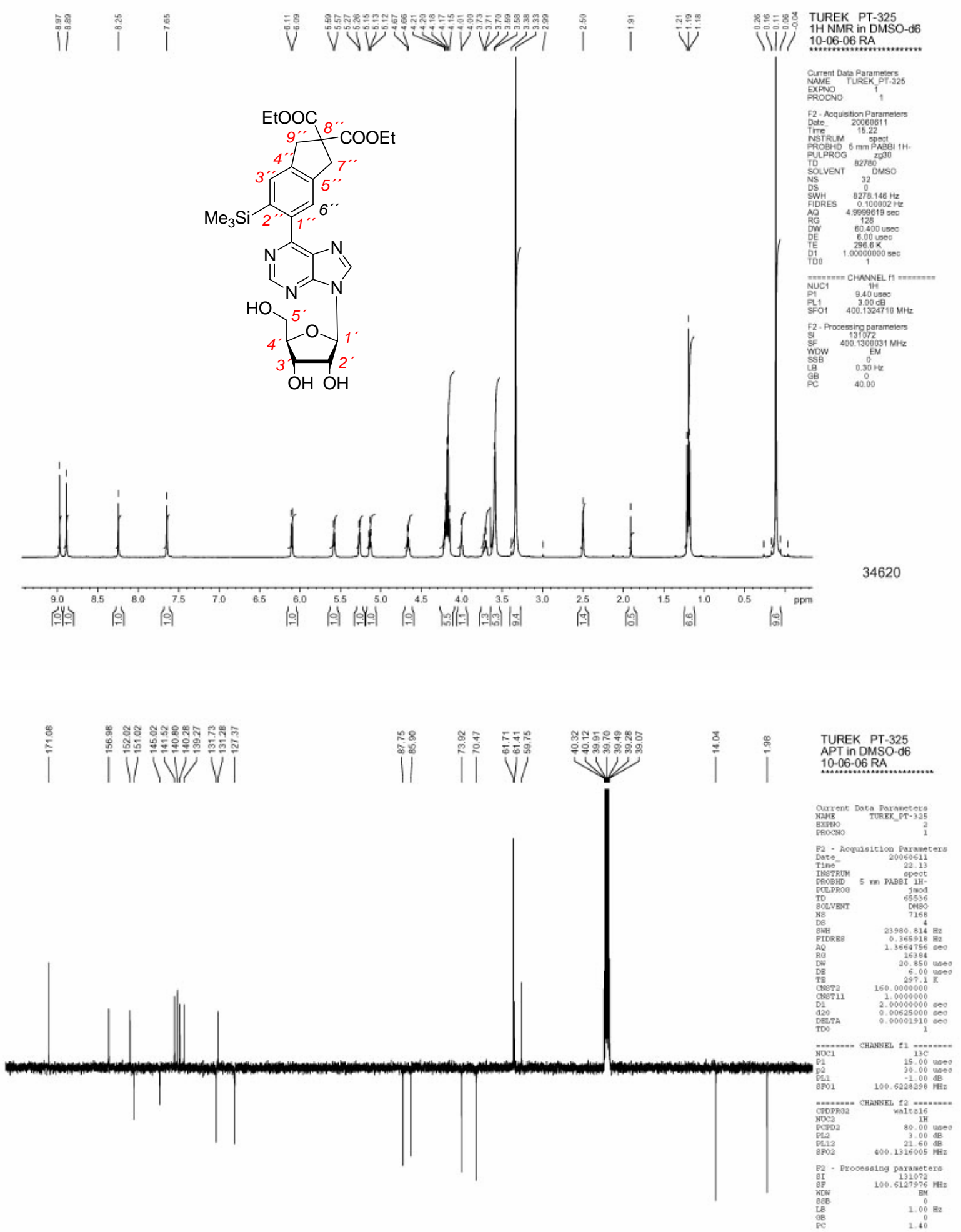